\title{
Dietary Patterns before and during Pregnancy and Risk of Gestational Diabetes Mellitus: A Systematic Review
}

The Pregnancy and Birth to 24 Months Project

Published date: April 15, 2019

Nutrition Evidence Systematic Review

Center for Nutrition Policy and Promotion

Food and Nutrition Service

U.S. Department of Agriculture

3101 Park Center Drive

Alexandria, Virginia 
This systematic review was conducted for the Pregnancy and Birth to 24 Months Project (P/B-24 Project) by the Nutrition Evidence Systematic Review (NESR) team at the Center for Nutrition Policy and Promotion, Food and Nutrition Service, USDA. All systematic reviews from the P/B-24 Project are available on the NESR website: https://nesr.usda.gov.

Conclusion statements drawn as part of this systematic review describe the state of science related to the specific question examined. Conclusion statements do not draw implications, and should not be interpreted as dietary guidance.

The contents of this document may be used and reprinted without permission. Endorsements by NESR, the Center for Nutrition Policy and Promotion, the Food and Nutrition Service, or the U.S. Department of Agriculture (USDA) of derivative products developed from this work may not be stated or implied.

In accordance with Federal civil rights law and USDA civil rights regulations and policies, the USDA, its Agencies, offices, and employees, and institutions participating in or administering USDA programs are prohibited from discriminating based on race, color, national origin, religion, sex, gender identity (including gender expression), sexual orientation, disability, age, marital status, family/parental status, income derived from a public assistance program, political beliefs, or reprisal or retaliation for prior civil rights activity, in any program or activity conducted or funded by USDA (not all bases apply to all programs). Remedies and complaint filing deadlines vary by program or incident.

Persons with disabilities who require alternative means of communication for program information (e.g., Braille, large print, audiotape, American Sign Language, etc.) should contact the responsible Agency or USDA's TARGET Center at (202) 720-2600 (voice and TTY) or contact USDA through the Federal Relay Service at (800) 877-8339. Additionally, program information may be made available in languages other than English.

To file a program discrimination complaint, complete the USDA Program Discrimination Complaint Form, AD3027, found online at How to File a Program Discrimination Complaint and at any USDA office or write a letter addressed to USDA and provide in the letter all of the information requested in the form. To request a copy of the complaint form, call (866) 632-9992. Submit your completed form or letter to USDA by: (1) mail: U.S. Department of Agriculture, Office of the Assistant Secretary for Civil Rights, 1400 Independence Avenue, SW, Washington, D.C. 20250-9410; (2) fax: (202) 690-7442; or (3) email: program.intake@usda.gov.

USDA is an equal opportunity provider, employer, and lender.

\section{Suggested citation for this systematic review:}

Nutrition Evidence Systematic Review Team and Pregnancy Technical Expert Collaborative. Dietary Patterns before and during Pregnancy and Risk of Gestational Diabetes: A Systematic Review. Pregnancy and Birth to 24 Months Project. Alexandria, VA: U.S. Department of Agriculture, Food and Nutrition Service, Center for Nutrition Policy and Promotion, April 2019. Available at: https://nesr.usda.gov/project-specific-overview-pb-24$\underline{0}$

This systematic review has also been published in the American Journal of Clinical Nutrition: Raghavan R, Dreibelbis C, Kingshipp BL, Wong YP, Abrams B, Gernand AD, et al. Dietary patterns before and during pregnancy and maternal outcomes: a systematic review. Am J Clin Nutr. 2019;109(7):705S-28S. doi: $10.1093 / a j c n / n q y 216$

Related citations are published in the American Journal of Clinical Nutrition:

- P/B-24 Project overview: Stoody EE, Spahn JM, Casavale KO. The Pregnancy and Birth to 24 Months Project: a series of systematic reviews on diet and health. Am J Clin Nutr. 2019;109(7):685S-97S. doi: 10.1093/ajcn/nqy372.

- P/B-24 systematic review methodology: Obbagy JE, Spahn JM, Wong YP, Psota TL, Spill MK, Dreibelbis C, et al. Systematic review methodology used in the Pregnancy and Birth to 24 Months Project. Am J Clin Nutr. 2019;109(7):698S-704S. doi: 10.1093/ajcn/nqy226

- Related systematic reviews from the P/B-24 Project: Raghavan R, Dreibelbis C, Kingshipp BL, Wong YP, Abrams B, Gernand AD, et al. Dietary patterns before and during pregnancy and birth outcomes: a systematic review. Am J Clin Nutr. 2019;109(7):729S-56S. doi: 10.1093/ajcn/nqy353 


\section{ACKNOWLEDGEMENTS}

\section{Pregnancy Technical Expert Collaborative (TEC) members:}

- Barbara Abrams, DrPH, RD, University of California, Berkeley School of Public Health

- Anne Bartholomew, MS, RD, USDA

- Lisa M. Bodnar, PhD, MPH, RD, University of Pittsburgh School of Public Health

- Alison Gernand, PhD, MPH, RD The Pennsylvania State University

- Kathleen Rasmussen, ScD, RD, Cornell University

- Anna Maria Siega-Riz, PhD, University of Virginia School of Nursing

- Jamie S. Stang, PhD, MPH, RDN, University of Minnesota School of Public Health

\section{Nutrition Evidence Systematic Review (NESR) team:}

- Ramkripa Raghavani, DrPH, MPH, MSc, Panum Group, Lead analyst

- Carol Dreibelbisi, MPH, Panum Group, Analyst

- Brittany L. Kingshippi, PhD, Panum Group, Analyst (01/2017-Present)

- Yat Ping Wong, MLS, MPH, USDA, Librarian

\section{Project Leads:}

- Eve Essery Stoody, PhD, USDA

- Joanne M. Spahn, MS, RDN, USDA

- Kellie O. Casavale, PhD, RD, HHS

Federal Expert Group (FEG)-Technical Expert Collaborative (TEC) Liaisons:

- Anne Bartholomew, MS, RD, USDA

All TEC and NESR team members, Project leads, and FEG-TEC liaisons participated in establishing the research questions, analytic framework, and study inclusion and exclusion criteria. RR, CD, and YPW developed and conducted the literature search. $R R, C D$, and BLK screened search results, identified studies for inclusion, extracted data, and assessed risk of bias for included studies. BA, AB, LMB, AG, KR, AMSR, JSS, EES, JMS, and KOC reviewed and provided substantive feedback on all systematic review materials, including the synthesis of the body of evidence, conclusion statement, and grade of the strength of the evidence. RR prepared this report and EES, JMS, and KOC provided oversight. All authors critically reviewed and approved the final report. The authors declare no conflicts of interest.

FUNDING SOURCE: United States Department of Agriculture, Food and Nutrition Service, Center for Nutrition Policy and Promotion, Alexandria, VA

' Under contract with the Food and Nutrition Service, United States Department of Agriculture. 


\section{TABLE OF CONTENTS}

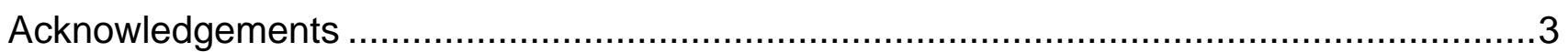

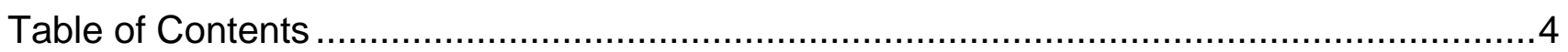

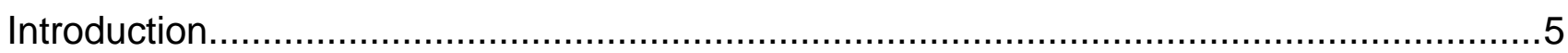

What is the relationship between dietary patterns before and during pregnancy and risk of

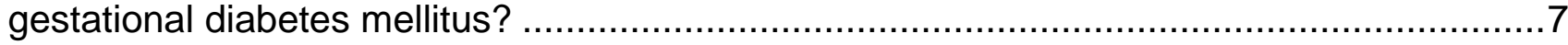

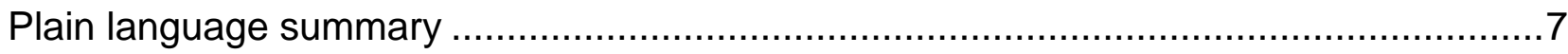

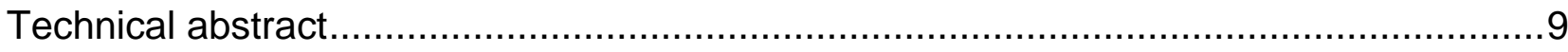

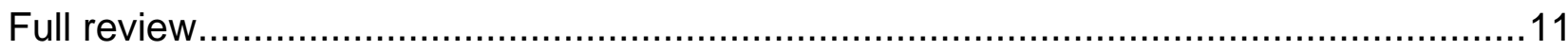

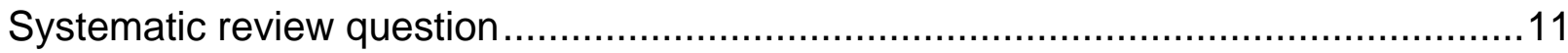

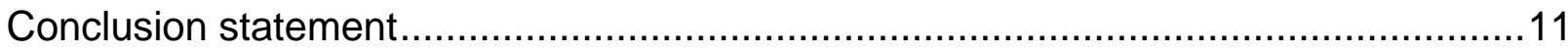

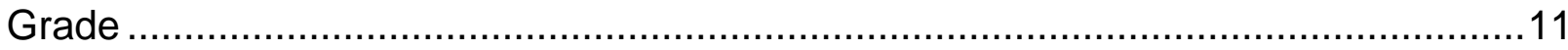

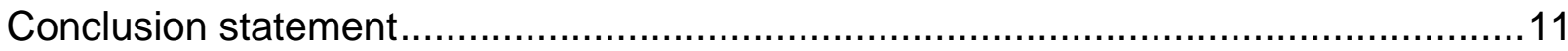

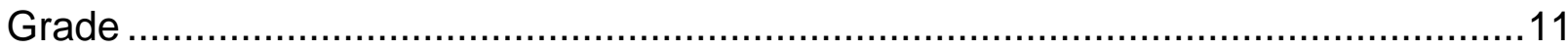

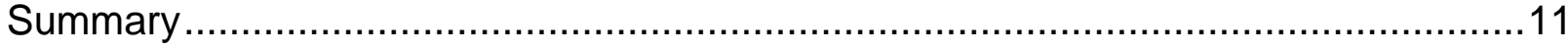

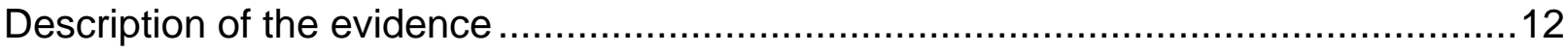

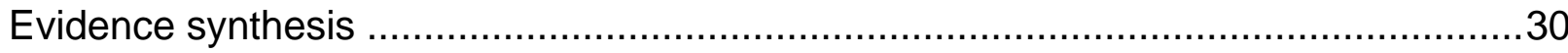

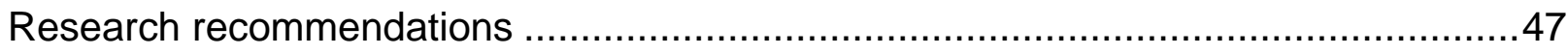

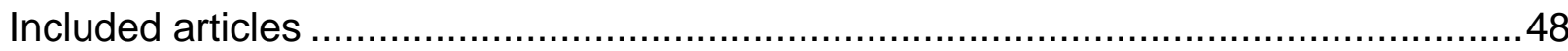

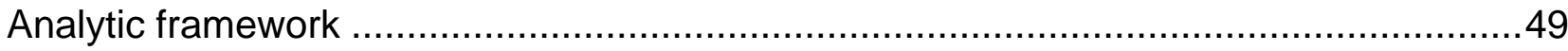

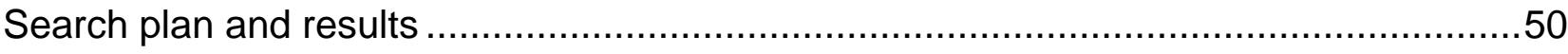

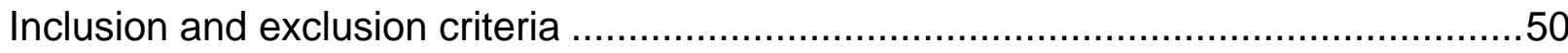

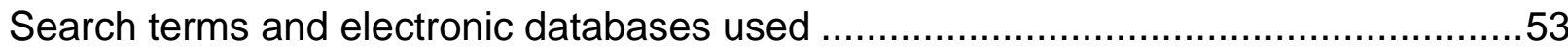

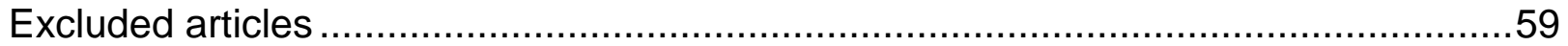

Table 1. Indices and scores used to assess the relationship between dietary patterns before

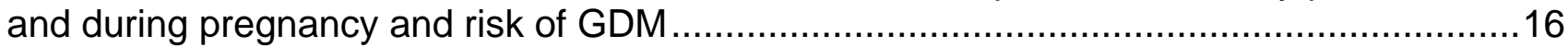
Table 2. Summary of dietary patterns identified using factor or principal component analysis

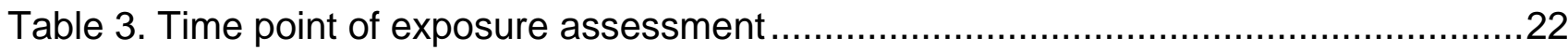

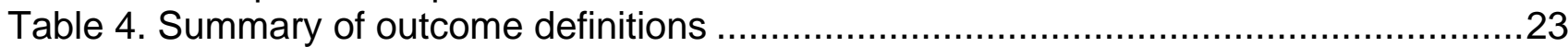

Table 5. Results grouped by methodology used for dietary pattern assessment ................34

Table 6. Inclusion and exclusion criteria ..........................................................................5

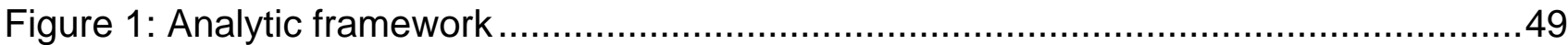

Figure 2: Flow chart of literature search and screening results ..................................58 


\section{INTRODUCTION}

This document describes a systematic review conducted to answer the following question: What is the relationship between dietary patterns before and during pregnancy and risk of gestational diabetes mellitus? This systematic review was conducted as part of the Pregnancy and Birth to 24 Months (P/B-24) Project by USDA's Nutrition Evidence Systematic Review (NESR).

The purpose of the P/B-24 Project was to conduct a series of systematic reviews on diet and health for women who are pregnant and for infants and toddlers from birth to 24 months of age. This project was a joint initiative led by USDA and HHS, and USDA's NESR carried out all of the systematic reviews. A Federal Expert Group (FEG), a broadly representative group of Federal researchers and program leaders, also provided input throughout the P/B-24 Project. More information about the P/B-24 Project has been publishedi and is available on the NESR website: https://nesr.usda.gov/project-specificoverview-pb-24-0.

NESR, formerly known as the Nutrition Evidence Library (NEL), specializes in conducting food- and nutrition-related systematic reviews using a rigorous, protocol-driven methodology. To conduct each P/B-24 systematic review, NESR's staff worked with a Technical Expert Collaborative (TEC), which is a group of 7-8 leading subject matter experts.

NESR's systematic review methodology involves developing and prioritizing systematic review questions, searching for and selecting studies, extracting and assessing the risk of bias of data from each included study, synthesizing the evidence, developing a conclusion statement, grading the evidence underlying the conclusion statement, and recommending future research. A detailed description of the methodology used in conducting systematic reviews for the P/B-24 Project has been publishediii and is available on the NESR website: https://nesr.usda.gov/pb-24-project-methodology-0. In addition, starting on page 49, this document includes details about the methodology as it was applied to the systematic review described herein. An analytic framework that illustrates the overall scope of the question, including the population, the interventions and/or exposures, comparators, and outcomes of interest, is found on page 49. In addition, the literature search plan that was used to identify studies included in this systematic review is found on page 50.

\footnotetext{
ii Stoody EE, Spahn JM, Casavale KO. The Pregnancy and Birth to 24 Months Project: a series of systematic reviews on diet and health. Am J Clin Nutr. 2019;109(7):685S-97S. doi: 10.1093/ajen/nqy372.

iii Obbagy JE, Spahn JM, Wong YP, Psota TL, Spill MK, Dreibelbis C, et al. Systematic review methodology used in the Pregnancy and Birth to 24 Months Project. Am J Clin Nutr. 2019;109(7):698S704S. doi: 10.1093/ajcn/nqy226.
} 


\section{List of abbreviations}

\begin{tabular}{ll}
\hline Abbreviation & Full name \\
\hline FEG & Federal Expert Group \\
\hline HHS & Department of Health and Human Services \\
\hline NEL & Nutrition Evidence Library \\
\hline NESR & Nutrition Evidence Systematic Review \\
\hline P/B-24 & Pregnancy and Birth to 24 Months Project \\
\hline TEC & Technical Expert Collaborative \\
\hline USDA & United States Department of Agriculture \\
\hline
\end{tabular}




\section{WHAT IS THE RELATIONSHIP BETWEEN DIETARY PATTERNS BEFORE AND DURING PREGNANCY AND RISK OF GESTATIONAL DIABETES MELLITUS?}

\section{PLAIN LANGUAGE SUMMARY}

What is the question?

- The question is: What is the relationship between dietary patterns before and during pregnancy and risk of gestational diabetes mellitus?

\section{What is the answer to the question?}

- Limited but consistent evidence suggests that certain dietary patterns before pregnancy are associated with a reduced risk of gestational diabetes mellitus. These protective dietary patterns are:

$\circ$ higher in vegetables, fruits, whole grains, nuts, legumes, and fish, and

- lower in red and processed meats.

- Most of the research was conducted in healthy, Caucasian women with access to health care.

- Evidence is insufficient to estimate the association between dietary patterns during pregnancy and risk of gestational diabetes mellitus.

Why was this question asked?

- This important public health question was identified and prioritized as part of the U.S. Department of Agriculture and Department of Health and Human Services Pregnancy and Birth to 24 Months Project.

How was this question answered?

- A team of Nutrition Evidence Systematic Review staff conducted a systematic review in collaboration with a group of experts called a Technical Expert Collaborative.

\section{What is the population of interest?}

- Women who are pregnant or able to become pregnant, ages 15-44 years.

\section{What evidence was found?}

- This review includes 11 studies that were published since 1980. Some studies were small, including 12 participants, while others were really large and included 15,254 participants. Most of these women were Caucasians that had access to health care. The extent of overweight and obesity varied. In some studies 1 in 10 women were overweight or obese, while in others 1 in 3 were overweight or obese.

- Studies used different approaches to develop dietary patterns:

- 5 studies used an existing index or score.

- 4 studies used data-driven methods such as principal components analysis.

- 1 study used both an index/score and a data-driven method.

- The randomized controlled trial used an experimental diet. 
- Studies showed that what women ate several years (2-10 y) before pregnancy was important for gestational diabetes risk.

- However, what women ate during pregnancy did not increase or decrease the gestational diabetes risk.

\section{How up-to-date is this review?}

- This review includes literature from $01 / 1980$ to $01 / 2017$. 


\section{TECHNICAL ABSTRACT}

\section{Background}

- This systematic review was conducted as part of the U.S. Department of Agriculture and Department of Health and Human Services Pregnancy and Birth to 24 Months Project.

- The goal of this systematic review was to examine the following question: What is the relationship between dietary patterns before and during pregnancy and risk of gestational diabetes mellitus?

\section{Conclusion Statement and Grades}

- Limited but consistent evidence suggests that certain dietary patterns before pregnancy are associated with a reduced risk of gestational diabetes mellitus. These protective dietary patterns are:

$\circ$ higher in vegetables, fruits, whole grains, nuts, legumes, and fish, and

- lower in red and processed meats.

- Most of the research was conducted in healthy, Caucasian women with access to health care.

Grade: Limited

- Evidence is insufficient to estimate the association between dietary patterns during pregnancy and risk of gestational diabetes mellitus.

Grade: Grade not assignable

\section{Methods}

- The systematic review was conducted by a team of staff from the Nutrition Evidence Systematic Review in collaboration with a Technical Expert Collaborative.

- Literature searches were conducted using PubMed, Embase, Cochrane, and other databases to identify studies that evaluated the relationship between dietary patterns before and during pregnancy and risk of gestational diabetes mellitus. A manual search was conducted to identify articles that may not have been included in the electronic databases searched. Articles were screened by two authors independently for inclusion based on pre-determined criteria. Those that met the following criteria were included in the review: randomized controlled trials (RCTs), prospective or retrospective cohort studies or nested case-control studies; studies enrolling human subjects who were pregnant women or women capable of becoming pregnant, healthy or at elevated chronic disease risk (only some, not all, could have a chronic or pregnancy-related condition), and between the ages of 15 and 44; subjects from countries with high or very high human development (2015 Human Development Index); and studies published in English in peer-reviewed journals.

- The date range was from January 1980 to January 2017. The intervention or exposure was dietary patterns before or during pregnancy measured via indices and scores, cluster or factor analysis, reduced rank regression, or other methods. The outcome was risk of GDM and related intermediate outcomes (glucose intolerance, insulin resistance, $\mathrm{HbA1C}$, HOMA-IR, fasting blood glucose, and oral glucose tolerance test). 
- Data from each included article were extracted, risks of bias were assessed, and both were checked for accuracy. The body of evidence was qualitatively synthesized, a conclusion statement was developed, and the strength of the evidence (grade) was assessed using pre-established criteria including evaluation of the internal validity/risk of bias, adequacy, consistency, impact, and generalizability of available evidence.

\section{Summary of Evidence}

- This systematic review includes 10 prospective cohort studies and 1 pilot RCT, published between 1998 and 2016.

- The studies used multiple approaches to assess dietary patterns. Five studies used indices/scores to assess dietary patterns, four studies used factor or principal component analysis (PCA) and one study used both an index/score and PCA. In addition, one RCT assigned subjects to one of two experimental diets.

- Overall, 8 of the 11 included studies found statistically significant associations between dietary patterns and GDM risk among healthy Caucasian women with access to health care. Greater adherence to a protective dietary pattern before and during pregnancy was associated with a decrease in GDM risk of $24 \%$ to $56 \%$. Higher adherence to a detrimental pattern was associated with an increase in risk of $23 \%$ to $63 \%$.

- There is heterogeneity in terms of when dietary data were assessed. Five studies measured diet before pregnancy while the rest $(n=6)$ assessed diet during pregnancy.

- Greater adherence to a healthy diet assessed 2-10 years before pregnancy showed a consistent inverse association with the risk of GDM in all the studies. These findings are also in agreement with the evidence linking dietary patterns and type 2 diabetes mellitus risk in non-pregnant populations.

- There were mixed findings in studies that assessed diet during pregnancy: 1) three studies showed an association with GDM, 2) one showed an inverse association with blood glucose, only, and not with GDM, 3) one showed an effect on blood glucose and insulin response but did not study GDM, and 4) one other study showed no association with GDM.

- Generalizability of the studies is limited to healthy Caucasian women who have access to health care. Minority women and those of lower SES are underrepresented in this body of evidence. 


\section{FULL REVIEW}

\section{Systematic review question}

What is the relationship between dietary patterns before and during pregnancy and risk of gestational diabetes mellitus?

\section{Conclusion statement}

Limited but consistent evidence suggests that certain dietary patterns before pregnancy are associated with a reduced risk of gestational diabetes mellitus. These protective dietary patterns are:

- $\quad$ higher in vegetables, fruits, whole grains, nuts, legumes, and fish, and

- lower in red and processed meats.

Most of the research was conducted in healthy, Caucasian women with access to health care.

\section{Grade}

Limited

\section{Conclusion statement}

Evidence is insufficient to estimate the association between dietary patterns during pregnancy and risk of gestational diabetes mellitus.

\section{Grade}

Grade not assignable

\section{Summary}

- This systematic review includes 10 prospective cohort studies and 1 pilot randomized controlled trial (RCT), published between 1998 and 2016.

- The studies used multiple approaches to assess dietary patterns. Five studies used indices/scores to assess dietary patterns, four studies used factor or principal component analysis (PCA) and one study used both an index/score and PCA. In addition, one RCT assigned subjects to one of two experimental diets.

- Overall, 8 of the 11 included studies found statistically significant associations between dietary patterns and GDM risk among healthy Caucasian women with access to health care. Greater adherence to a protective dietary pattern before and during pregnancy was inversely associated with a decrease in GDM risk of $24 \%$ to $56 \%$. Higher adherence to a detrimental pattern was positively associated with an increase in risk of $23 \%$ to $63 \%$.

- There is heterogeneity in terms of when dietary data were assessed. Five studies measured diet before pregnancy while the rest $(n=6)$ assessed diet during pregnancy.

- Greater adherence to a healthy diet assessed 2-10 years before pregnancy showed a consistent inverse association with the risk of gestational diabetes mellitus (GDM) in all five studies. These findings were also in agreement with the evidence linking dietary patterns and diabetes mellitus risk in non-pregnant populations. 
- There were mixed findings in studies that assessed diet during pregnancy: three studies showed an association with GDM, one showed an inverse association only with blood glucose and not with GDM, one showed an effect on blood glucose and insulin response but did not study GDM, and one other study showed no association.

- Generalizability of the studies is limited to healthy Caucasian women who have access to health care. Minority women and those of lower SES are underrepresented in this body of evidence.

\section{Description of the evidence}

- The search included articles from very high and high Human Development Index (HDI) countries, and the search timeframe spanned between January 1980 and January 2017.

- This evidence review includes 10 prospective cohort studies and 1 pilot RCT that examined the relationship between dietary patterns before and during pregnancy and risk of GDM (1-11).

- Only three studies were conducted in a cohort or a trial that was originally designed to assess the relationship between maternal diet and risk of GDM (1, $3,9)$. Other studies assessed exposures and outcomes as part of secondary objectives/analysis.

- Of the 11 studies, three used Nurses' Health Study II (NHS II) cohort data (8, $10,11)$, two used Australian Longitudinal Study on Women's Health cohort data $(6,7)$, two used Project Viva cohort data $(4,5)$, one used Gestational Diabetes in the Mediterranean Region Study data (3), and one used Born in Guangzhou Cohort Study data (2). Two were individual studies $(1,9)$.

- About half of the studies ( $n=6)$ (including one RCT, three NHS II and two Project Viva) were conducted in the U.S $(1,4,5,8,10,11)$. Other countries in which studies were conducted include Australia (6,7), China (2) and Iceland (9). In addition, a multi-country study was conducted in the following countries: Algeria, France, Greece, Italy, Lebanon, Malta, Morocco, Serbia, Syria and Tunisia (3). See the map below.

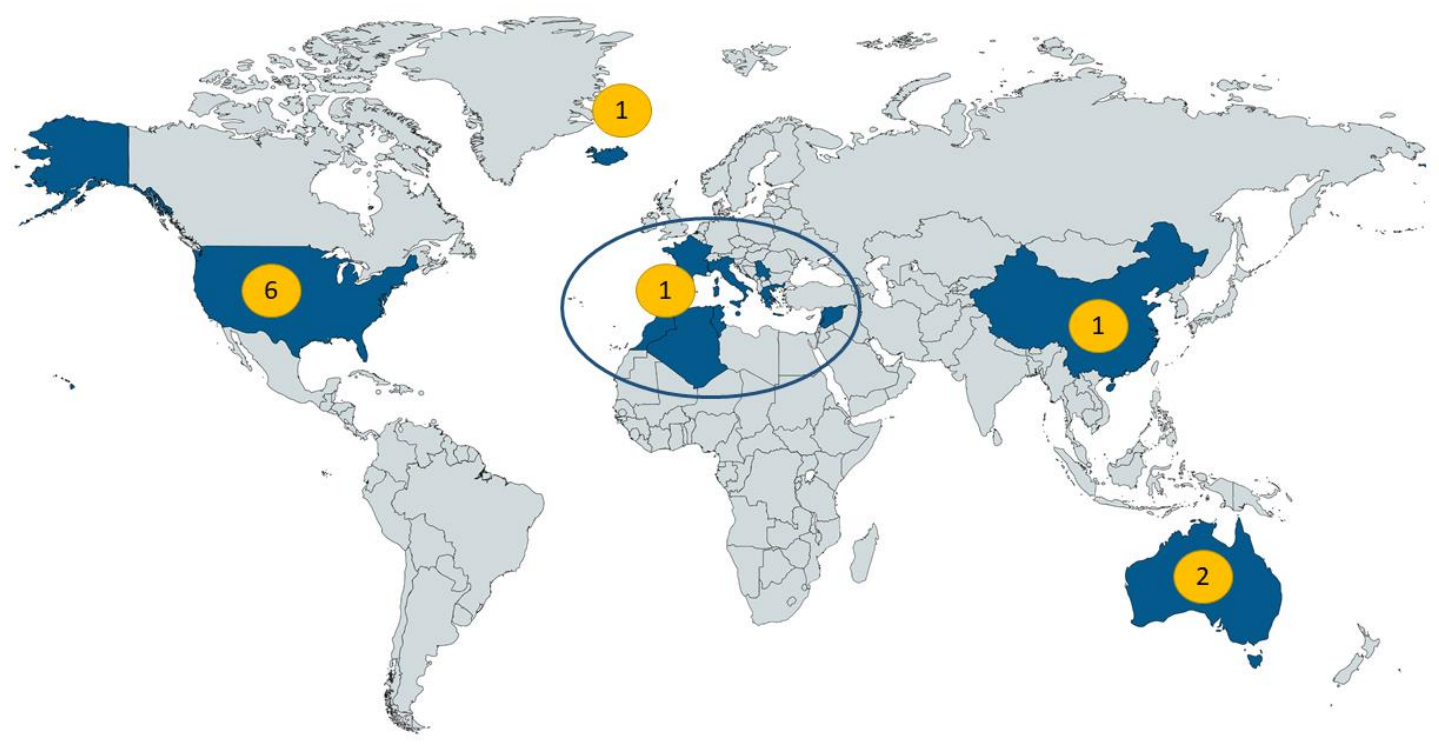




\section{Subject characteristics:}

- Sample size of the studies ranged from 12 subjects in the RCT (1) to 15,254 subjects in the NHS II (8), with a median sample size of 3,063.

- Age: Many studies included women between 20 and 40 years. Two cohorts (NHS II and Project Viva) included women outside of this age range $(<20$ years and $\geq 40$ years), but the corresponding studies did not specify the number/percentage of women who were outside this range.

- Singleton vs. multiple births: Six studies included only singleton pregnancies $(2,4,5,8,10,11)$. The rest of the studies did not report these characteristics $(1$, $3,6,7,9)$.

- Health Characteristics: A majority of the studies $(n=9)$ excluded participants with a history of GDM or Type 1 or Type 2 diabetes. Only two studies included women with a history of $\operatorname{GDM}(3,4)$, with Radesky reporting a prevalence of $2 \%$. At least one study reported excluding women with polycystic ovary syndrome (PCOS) (4), whereas another study reported a PCOS prevalence of $7 \%(6)$. Only $\mathrm{He}$ et al. reported including participants with a family history of diabetes and the prevalence was 19\% (2).

- Smoking during pregnancy varied across studies.

- The prevalence of reported smoking during pregnancy among study participants was between 3\% (4) and 8\% (11).

- Schoenaker et al. reported a pre-pregnancy smoking status of $20 \%(6)$, and these data were collected 3-9 years prior to becoming pregnant.

- One study excluded smokers (9), and seven studies did not report smoking status $(1-3,5,7,8,10)$.

- Race/ethnicity: More than half of the studies $(n=7)$ did not report participants' race/ethnicity. Studies using NHS II data had a high percentage of white women $(>90 \%)(8,11)$. Similarly, studies using Project Viva data $(4,5)$ reported that a majority of participants were white women $(72 \%)$.

- Parity: Most of the studies $(n=10)$ included data on parity, although the percentage of nulliparous vs. multiparous women was highly variable. The NHS II reported that about a third of women were nulliparous at baseline $(8,10)$, whereas the Chinese study reported that $93 \%$ were nulliparous (2). Since NHS II and ALSWH were longitudinal, some women contributed more than one pregnancy to the study.

- Pre-pregnancy BMI: The percentage of subjects with self-reported prepregnancy $\mathrm{BMI} \geq 30$ in individual studies ranged from $\sim 7 \%$ (7) to $23 \%$ (9). Some studies reported only the mean pre-pregnancy BMI (Range: 23.9 to 24.6 $\mathrm{kg} / \mathrm{m}^{2}$ ). The RCT did not report BMI (1).

- Maternal education: All participants in the NHS II were nurses $(8,10,11)$. Apart from three studies that did not report on maternal education $(1,3,9)$, mothers with undergraduate degrees or higher ranged from $~ 55 \%$ to $69 \%$.

- Socioeconomic status was not reported in a majority of studies. In Project Viva studies, $12 \%-13 \%$ of the participants had a household income $<\$ 40,000$ $(4,5)$.

\section{Interventions/Exposures:}

Dietary patterns were assessed using index/score analysis and factor and principal components analysis (PCA); one study randomly assigned participants to one of two 
experimental diets. A description of the studies included by method used to measure dietary patterns is included below.

- Index/score analysis (Table 1. Indices and scores used to assess the relationship between dietary patterns before and during pregnancy and risk of GDM): Six studies included in this review used one or more of the following indices/scores:

- Alternate Healthy Eating Index (aHEI) (8)

- Alternate HEl for Pregnancy (AHEI-P) (5)

- Alternate Mediterranean (aMED) (8)

- Dietary Approaches to Stop Hypertension (DASH) (8)

- Healthy Eating Index (HEI) (9)

- Mediterranean Diet Score $(3,7)$

- Modified Alternate Healthy Eating Index-2010 (11)

- Factor analysis and principal component analysis (Table 2. Summary of dietary patterns identified using factor or principal component analysis): Five studies included in this review assessed dietary patterns using factor or PCA $(2,4,6,9,10)$.

- Experimental diet: The pilot RCT in this body of evidence randomly assigned participants to one of two experimental diets (1):

- Aboriginal carbohydrate diet (Low-Gl): used carbohydrates from unprocessed whole grains, fruits, beans, vegetables, and many dairy products; includes most dense whole grain and multigrain breads, bran cereals, pastas, fresh fruits and vegetables, yogurt, ice cream, and nuts

- Cafeteria carbohydrate diet (High-Gl): used carbohydrates from highly processed grains, root vegetables, and simple sugars; includes many highly-refined breads, potatoes, instant rice, most breakfast cereals, desserts, and snack-type foods

\section{Time point of exposure (Table 3. Time point of exposure assessment):}

- Radesky et al. and Rifas-Shiman et al. used data from Project Viva and therefore had similar data collection protocols. The food frequency questionnaire (FFQ) was administered during the first trimester ( 11 weeks), and the recall period reflected the time of conception until the time the FFQ was assessed. Project Viva also collected FFQ data between 26-28 weeks. While Rifas-Shiman et al. presented GDM outcomes pertaining to the second trimester exposure, this body of evidence excluded the second trimester exposure. This decision was made because some women might have known about their GDM status prior to the FFQ administration, thereby potentially impacting temporality (and this is consistent with Radesky et al.'s approach).

- Two studies collected dietary data during the second trimester (between 19 and 27 weeks) $(2,9)$. For both of these studies the recall period was relatively short ( $<1$ week). Although the studies reported overlapping time periods of exposure and outcome assessment, both of these articles clarified that exposure assessment preceded outcome assessment.

- Karamanos et al. reported gathering dietary data prior to performing the OGTT (24-32 weeks); however, it is unclear exactly when the dietary data were collected and what recall period was reflected.

- Studies from the NHS II and ALSWH used dietary data that were collected 
between 2 and 10 years before pregnancy.

- The RCT in this body of evidence assigned women to experimental diets at 8 weeks and followed them throughout pregnancy 
Table 1. Indices and scores used to assess the relationship between dietary patterns before and during pregnancy and risk of GDM

\begin{tabular}{|c|c|c|c|c|c|c|c|c|}
\hline $\begin{array}{l}\text { Index/ Score } \\
\text { (Reference) }\end{array}$ & $\begin{array}{l}\text { Med. Diet } \\
\text { score }^{1} \\
\text { (min-max } \\
\text { score) }\end{array}$ & $\begin{array}{c}\text { Alternate } \\
\text { HEI-P } \\
\text { (min-max } \\
\text { score) }\end{array}$ & $\begin{array}{l}\text { Med. Diet } \\
\text { score } \\
\text { (min-max } \\
\text { score) }\end{array}$ & $\begin{array}{l}\text { aMED } \\
\text { (min-max } \\
\text { score) }\end{array}$ & $\begin{array}{l}\text { DASH }^{5} \\
\text { (min-max } \\
\text { score) }\end{array}$ & $\begin{array}{c}\text { aHEl } \\
\text { (min-max } \\
\text { score) }\end{array}$ & $\begin{array}{c}\text { HEl }^{7} \\
\text { (min-max } \\
\text { score) }\end{array}$ & $\begin{array}{c}\text { Alternate } \\
\text { HEl-2010 } \\
\text { (min-max } \\
\text { score) }\end{array}$ \\
\hline Article & $\begin{array}{l}\text { Karamanos, } \\
2014\end{array}$ & $\begin{array}{l}\text { Rifas- } \\
\text { Shiman et } \\
\text { al., } 2009\end{array}$ & $\begin{array}{l}\text { Schoenaker, } \\
2016\end{array}$ & $\begin{array}{l}\text { Tobias, } \\
2012\end{array}$ & $\begin{array}{l}\text { Tobias, } \\
2012\end{array}$ & $\begin{array}{l}\text { Tobias, } \\
2012\end{array}$ & $\begin{array}{l}\text { Tryggvadottir, } \\
2016\end{array}$ & $\begin{array}{l}\text { Zhang, } \\
2014\end{array}$ \\
\hline Component & $\begin{array}{l}\text { Total score: } \\
0-12\end{array}$ & $\begin{array}{l}\text { Total } \\
\text { score: 0-90 }\end{array}$ & $\begin{array}{l}\text { Total score: } \\
0-10\end{array}$ & $\begin{array}{l}\text { Total score: } \\
0 \text { to } 8\end{array}$ & $\begin{array}{l}\text { Total } \\
\text { score: } \\
8 \text { to } 39\end{array}$ & $\begin{array}{l}\text { Total score: } \\
2.5 \text { to } 87.5\end{array}$ & $\begin{array}{l}\text { Total score: } \\
8-800\end{array}$ & $\begin{array}{l}\text { Total } \\
\text { score: } \\
2.5-77.5\end{array}$ \\
\hline Vegetables & $\begin{array}{l}\text { Vegetables } \\
\geq \text { Median=1; } \\
<\text { Median=0 }\end{array}$ & $\begin{array}{l}\text { Vegetables } \\
(0-10) \\
\text { (Includes } \\
\text { tofu and } \\
\text { soybeans) }\end{array}$ & $\begin{array}{l}\text { Vegetables } \\
\geq \text { Median=1; } \\
<\text { Median }=0\end{array}$ & $\begin{array}{l}\text { Vegetables } \\
\geq \text { Median }=1 ; \\
<\text { Median }=0\end{array}$ & $\begin{array}{l}\text { Vegetables } \\
(1-5)\end{array}$ & $\begin{array}{l}\text { Vegetables } \\
(0-10)\end{array}$ & $\begin{array}{l}\text { Vegetables } \\
(1-100)\end{array}$ & $\begin{array}{l}\text { Vegetables } \\
(0-10)\end{array}$ \\
\hline
\end{tabular}

\footnotetext{
1 Trichopoulou, A., Kouris-Blazos, A., Wahlqvist, M. L., Gnardellis, C., Lagiou, P., Polychronopoulos, E., . . Trichopoulos, D. (1995). Diet and overall survival in elderly people. BMJ, 311(7018), 1457-1460.

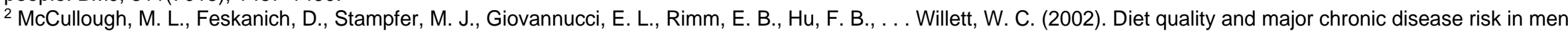
and women: moving toward improved dietary guidance. Am J Clin Nutr, 76(6), 1261-1271.

3 Trichopoulou, A., Costacou, T., Bamia, C., \& Trichopoulos, D. (2003). Adherence to a Mediterranean diet and survival in a Greek population. N Engl J Med, 348(26), 2599-2608. doi:10.1056/NEJMoa025039

${ }^{4}$ Fung, T. T., McCullough, M. L., Newby, P. K., Manson, J. E., Meigs, J. B., Rifai, N., . . Hu, F. B. (2005). Diet-quality scores and plasma concentrations of markers of inflammation and endothelial dysfunction. Am J Clin Nutr, 82(1), 163-173.

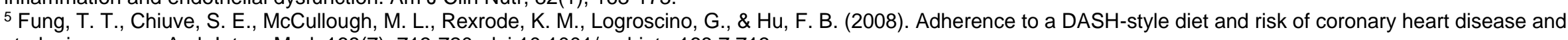
stroke in women. Arch Intern Med, 168(7), 713-720. doi:10.1001/archinte.168.7.713

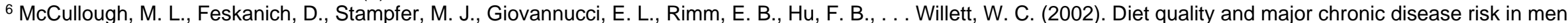
and women: moving toward improved dietary guidance. Am J Clin Nutr, 76(6), 1261-1271.

7 Icelandic Directorate of Health. (2015). Dietary guidelines for adults and children from the age of two. Retrieved from

http://www.landlaeknir.is/servlet/file/store93/item25796/radleggingar-um-mataraedi-2015.pdf

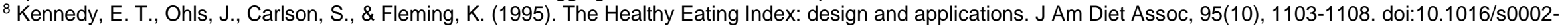
$8223(95) 00300-2$
} 


\begin{tabular}{|c|c|c|c|c|c|c|c|c|}
\hline $\begin{array}{l}\text { Index/ Score } \\
\text { (Reference) }\end{array}$ & $\begin{array}{l}\text { Med. Diet } \\
\text { score }^{1} \\
\text { (min-max } \\
\text { score) }\end{array}$ & $\begin{array}{l}\text { Alternate } \\
\text { HEl-P2 } \\
\text { (min-max } \\
\text { score) }\end{array}$ & $\begin{array}{l}\text { Med. Diet } \\
\text { score }^{3} \\
\text { (min-max } \\
\text { score) }\end{array}$ & $\begin{array}{c}\text { aMED } \\
\text { (min-max } \\
\text { score) }\end{array}$ & $\begin{array}{l}\text { DASH }^{5} \\
\text { (min-max } \\
\text { score) }\end{array}$ & $\begin{array}{c}\text { aHEI } \\
\text { (min-max } \\
\text { score) }\end{array}$ & $\begin{array}{c}\text { HEl }^{7} \\
\text { (min-max } \\
\text { score) }\end{array}$ & $\begin{array}{c}\text { Alternate } \\
\text { HEl-2010 } \\
\text { (min-max } \\
\text { score) }\end{array}$ \\
\hline & $\begin{array}{l}\text { Potatoes } \\
\geq \text { Median=1; } \\
<\text { Median=0 }\end{array}$ & & & & & & & \\
\hline \multirow[t]{2}{*}{$\begin{array}{l}\text { Fruit and/or } \\
\text { nuts }\end{array}$} & $\begin{array}{l}\text { Fruits } \\
\geq \text { Median=1; } \\
<\text { Median=0 }\end{array}$ & $\begin{array}{l}\text { Fruits } \\
(0-10)\end{array}$ & $\begin{array}{l}\text { Fruit and } \\
\text { nuts } \\
\geq \text { Median=1; } \\
<\text { Median=0 }\end{array}$ & $\begin{array}{l}\text { Fruits } \\
\geq \text { Median=1; } \\
<\text { Median=0 }\end{array}$ & $\begin{array}{l}\text { Fruits } \\
(1-5)\end{array}$ & $\begin{array}{l}\text { Fruits } \\
(0-10)\end{array}$ & $\begin{array}{l}\text { Fruits } \\
(1-100)\end{array}$ & $\begin{array}{l}\text { Fruit } \\
(0-10)\end{array}$ \\
\hline & & & & $\begin{array}{l}\text { Nuts } \\
\text { included } \\
\text { along with } \\
\text { legumes }\end{array}$ & $\begin{array}{l}\text { Nuts } \\
\text { included } \\
\text { along with } \\
\text { legumes }\end{array}$ & $\begin{array}{l}\text { Nuts } \\
\text { included } \\
\text { along with } \\
\text { legumes }\end{array}$ & $\begin{array}{l}\text { Nuts and } \\
\text { Seeds } \\
(1-100)\end{array}$ & $\begin{array}{l}\text { Nuts } \\
(0-10)\end{array}$ \\
\hline \multirow[t]{2}{*}{$\begin{array}{l}\text { Cereals/ } \\
\text { Grains and } \\
\text { whole grains }\end{array}$} & $\begin{array}{l}\text { Bread } \\
\geq \text { Median=1; } \\
<\text { Median=0 }\end{array}$ & blank & $\begin{array}{l}\text { Cereals } \\
\geq \text { Median }=1 ; \\
<\text { Median }=0\end{array}$ & $\begin{array}{l}\text { Whole } \\
\text { grains } \\
\geq \text { Median=1; } \\
<\text { Median=0 }\end{array}$ & $\begin{array}{l}\text { Whole } \\
\text { grains } \\
(1-5)\end{array}$ & $\begin{array}{l}\text { Cereal } \\
\text { Fiber } \\
(0-10)\end{array}$ & $\begin{array}{l}\text { Unground/ } \\
\text { Wholeground } \\
\text { cereals } \\
(1-100)\end{array}$ & $\begin{array}{l}\text { Whole- } \\
\text { grains } \\
(0-10)\end{array}$ \\
\hline & $\begin{array}{l}\text { Cereals } \\
\geq \text { Median=1; } \\
<\text { Median }=0\end{array}$ & & & & & & & \\
\hline Legumes & $\begin{array}{l}\text { Legumes } \\
\geq \text { Median=1; } \\
<\text { Median=0 }\end{array}$ & & $\begin{array}{l}\text { Legumes } \\
\geq \text { Median=1; } \\
<\text { Median=0 }\end{array}$ & $\begin{array}{l}\text { Nuts, } \\
\text { legumes, } \\
\text { soy } \\
\text { >Median=1; } \\
\text { <Median=0 }\end{array}$ & $\begin{array}{l}\text { Nuts, } \\
\text { legumes, } \\
\text { soy } \\
(1-5)\end{array}$ & $\begin{array}{l}\text { Nuts, } \\
\text { legumes, } \\
\text { soy } \\
(0-10)\end{array}$ & & \\
\hline
\end{tabular}




\begin{tabular}{|c|c|c|c|c|c|c|c|c|}
\hline $\begin{array}{l}\text { Index/ Score } \\
\text { (Reference) }\end{array}$ & $\begin{array}{l}\text { Med. Diet } \\
\text { score }^{1} \\
\text { (min-max } \\
\text { score) }\end{array}$ & $\begin{array}{l}\text { Alternate } \\
\text { HEI-P } \\
\text { (min-max } \\
\text { score) }\end{array}$ & $\begin{array}{l}\text { Med. Diet } \\
\text { score }^{3} \\
\text { (min-max } \\
\text { score) }\end{array}$ & $\begin{array}{l}\text { aMED } \\
\text { (min-max } \\
\text { score) }\end{array}$ & $\begin{array}{l}\text { DASH }^{5} \\
\text { (min-max } \\
\text { score) }\end{array}$ & $\begin{array}{c}\text { aHEI }^{6} \\
\text { (min-max } \\
\text { score) }\end{array}$ & $\begin{array}{c}\text { HEI }^{7} \\
\text { (min-max } \\
\text { score) }\end{array}$ & $\begin{array}{c}\text { Alternate } \\
\text { HEI-2010 } \\
\text { (min-max } \\
\text { score) }\end{array}$ \\
\hline \multirow[t]{2}{*}{ Meat } & $\begin{array}{l}\text { Meat } \\
\geq \text { Median=0; } \\
<\text { Median=1 } \\
\text { (reverse } \\
\text { scored) }\end{array}$ & $\begin{array}{l}\text { Ratio of } \\
\text { white } \\
\text { (poultry/ } \\
\text { fish) to red } \\
\text { meat } \\
\text { (beef, } \\
\text { pork, lamb, } \\
\text { processed) } \\
(0-10)\end{array}$ & $\begin{array}{l}\text { Meat } \\
\geq \text { Median=0; } \\
<\text { Median=1 } \\
\text { (reverse } \\
\text { scored) }\end{array}$ & $\begin{array}{l}\text { Red and } \\
\text { processed } \\
\text { meats } \\
\geq \text { Median=0; } \\
<\text { Median=1 } \\
\text { (reverse } \\
\text { scored) }\end{array}$ & $\begin{array}{l}\text { Red and } \\
\text { processed } \\
\text { meats } \\
(1-5)\end{array}$ & $\begin{array}{l}\text { White:Red } \\
\text { meat ratio } \\
(0-10)\end{array}$ & & $\begin{array}{l}\text { Red and } \\
\text { processed } \\
\text { meat } \\
(0-10) \\
\text { (reverse } \\
\text { scored) }\end{array}$ \\
\hline & & $\begin{array}{l}\text { Excluded } \\
\text { nuts and } \\
\text { soy }\end{array}$ & $\begin{array}{l}\text { Poultry } \\
\geq \text { Median=0; } \\
<\text { Median=1 } \\
\text { (reverse } \\
\text { scored) }\end{array}$ & & & & & \\
\hline \multirow[t]{2}{*}{$\begin{array}{l}\text { Fish and } \\
\text { other protein } \\
\text { foods }\end{array}$} & Fish & $\begin{array}{l}\text { Included } \\
\text { with meat }\end{array}$ & $\begin{array}{l}\text { Fish } \\
\geq \text { Median=1; } \\
<\text { Median=0 }\end{array}$ & $\begin{array}{l}\text { Fish and } \\
\text { seafood } \\
\geq \text { Median=1; } \\
<\text { Median=0 }\end{array}$ & & & $\begin{array}{l}\text { Fish and } \\
\text { seafood } \\
(1-100)\end{array}$ & \\
\hline & $\begin{array}{l}\text { Eggs } \\
\geq \text { Median=0; } \\
<\text { Median=1 } \\
\text { (reverse } \\
\text { scored) }\end{array}$ & & & & & & & \\
\hline
\end{tabular}




\begin{tabular}{|c|c|c|c|c|c|c|c|c|}
\hline $\begin{array}{l}\text { Index/ Score } \\
\text { (Reference) }\end{array}$ & $\begin{array}{l}\text { Med. Diet } \\
\text { score }^{1} \\
\text { (min-max } \\
\text { score) }\end{array}$ & $\begin{array}{l}\text { Alternate } \\
\text { HEl-P2 } \\
\text { (min-max } \\
\text { score) }\end{array}$ & $\begin{array}{l}\text { Med. Diet } \\
\text { score }^{3} \\
\text { (min-max } \\
\text { score) }\end{array}$ & $\begin{array}{l}\text { aMED } \\
\text { (min-max } \\
\text { score) }\end{array}$ & $\begin{array}{l}\text { DASH }^{5} \\
\text { (min-max } \\
\text { score) }\end{array}$ & $\begin{array}{c}\text { aHEI } \\
\text { (min-max } \\
\text { score) }\end{array}$ & $\begin{array}{c}\text { HEI }^{7} \\
\text { (min-max } \\
\text { score) }\end{array}$ & $\begin{array}{c}\text { Alternate } \\
\text { HEl-2010 } \\
\text { (min-max } \\
\text { score) }\end{array}$ \\
\hline \multirow[t]{2}{*}{ Dairy } & $\begin{array}{l}\text { Dairy } \\
\text { products } \\
\geq \text { Median=0; } \\
\text { <Median=1 } \\
\text { (reverse } \\
\text { scored) }\end{array}$ & & $\begin{array}{l}\text { High-fat } \\
\text { dairy } \\
\geq \text { Median=0; } \\
\text { <Median=1 } \\
\text { (reverse } \\
\text { scored) }\end{array}$ & & $\begin{array}{l}\text { Low-fat } \\
\text { dairy } \\
(1-5)\end{array}$ & & & \\
\hline & $\begin{array}{l}\text { Cheese } \\
\geq \text { Median=0; } \\
<\text { Median=1 }\end{array}$ & & & & & & & \\
\hline \multirow[t]{3}{*}{ Fat } & $\begin{array}{l}\text { Ratio of } \\
\text { olive oil to } \\
\text { animal fat } \\
\geq \text { Median=1; } \\
<\text { Median=0 }\end{array}$ & $\begin{array}{l}\text { trans fat } \\
(0-10) \\
(\text { reverse } \\
\text { scored) }\end{array}$ & $\begin{array}{l}M: S^{9} \text { fat } \\
\text { ratio } \\
\geq \text { Median=1; } \\
<\text { Median=0 }\end{array}$ & $\begin{array}{l}M: S^{10} \text { fat } \\
\text { ratio } \\
\geq \text { Median=1; } \\
<\text { Median=0 }\end{array}$ & & $\begin{array}{l}\text { trans fat } \\
(0-10) \\
\text { (reverse } \\
\text { scored) }\end{array}$ & & $\begin{array}{l}\text { trans fat } \\
(0-10) \\
\text { (reverse } \\
\text { scored) }\end{array}$ \\
\hline & & $\begin{array}{l}\text { PUFA:SFA } \\
\text { ratio } \\
(0-10)\end{array}$ & & & & $\begin{array}{l}\text { PUFA:SFA } \\
(0-10)\end{array}$ & & $\begin{array}{l}\text { PUFA } \\
(0-10)\end{array}$ \\
\hline & & & & & & & & $\begin{array}{l}\text { Long chain } \\
\text { omega } 3 \\
\text { fatty acids } \\
(0-10)\end{array}$ \\
\hline Fiber & & $\begin{array}{l}\text { Fiber } \\
(0-10)\end{array}$ & & & & & & \\
\hline
\end{tabular}

${ }^{9}$ Monounsaturated fat: saturated fat

${ }^{10}$ Monounsaturated fat: saturated fat 


\begin{tabular}{|c|c|c|c|c|c|c|c|c|}
\hline $\begin{array}{l}\text { Index/ Score } \\
\text { (Reference) }\end{array}$ & $\begin{array}{l}\text { Med. Diet } \\
\text { score }^{1} \\
\text { (min-max } \\
\text { score) }\end{array}$ & $\begin{array}{l}\text { Alternate } \\
\text { HEl-P } \\
\text { (min-max } \\
\text { score) }\end{array}$ & $\begin{array}{l}\text { Med. Diet } \\
\text { score }^{3} \\
\text { (min-max } \\
\text { score) }\end{array}$ & $\begin{array}{l}\text { aMED }^{4} \\
\text { (min-max } \\
\text { score) }\end{array}$ & $\begin{array}{l}\text { DASH }^{5} \\
\text { (min-max } \\
\text { score) }\end{array}$ & $\begin{array}{c}\text { aHEl }^{6} \\
\text { (min-max } \\
\text { score) }\end{array}$ & $\begin{array}{c}\text { HEl }^{7} \\
\text { (min-max } \\
\text { score) }\end{array}$ & $\begin{array}{c}\text { Alternate } \\
\text { HEl-2010 } \\
\text { (min-max } \\
\text { score) }\end{array}$ \\
\hline \multirow[t]{3}{*}{ Nutrients } & & $\begin{array}{l}\text { Folate } \\
(0-10)\end{array}$ & & & & $\begin{array}{l}\text { Multivitamin } \\
\text { use } \\
(0-10)\end{array}$ & $\begin{array}{l}\text { Vitamin D } \\
(1-100)\end{array}$ & \\
\hline & & $\begin{array}{l}\text { Calcium } \\
(0-10)\end{array}$ & & & & & & \\
\hline & & $\begin{array}{l}\text { Iron } \\
(0-10)\end{array}$ & & & & & & \\
\hline Alcohol & & $\begin{array}{l}\text { Excluded } \\
\text { alcohol }\end{array}$ & $\begin{array}{l}\text { Alcohol } \\
\text { (score of } 1 \\
\text { for } 5-15 \mathrm{~g} / \mathrm{d} \text { ) }\end{array}$ & $\begin{array}{l}\text { Alcohol } \\
\text { (score of } 1 \\
\text { for } 5-15 \\
\mathrm{~g} / \mathrm{d} \text { ) }\end{array}$ & & $\begin{array}{l}\text { Moderate } \\
\text { alcohol }\end{array}$ & & $\begin{array}{l}\text { Excluded } \\
\text { alcohol }\end{array}$ \\
\hline Oils & & & & & & & $\begin{array}{l}\text { Vegetable } \\
\text { oils } \\
(1-100)\end{array}$ & \\
\hline Sodium & & & & & $\begin{array}{l}\text { Sodium } \\
(1-5) \\
\text { (reverse } \\
\text { scored) }\end{array}$ & & & $\begin{array}{l}\text { Sodium } \\
(0-10) \\
\text { (reverse } \\
\text { scored) }\end{array}$ \\
\hline $\begin{array}{l}\text { Soft Drinks/ } \\
\text { Sugar } \\
\text { sweetened } \\
\text { beverages }\end{array}$ & & & & & $\begin{array}{l}\text { Sweetened } \\
\text { Beverages } \\
(1-5) \\
\text { (reverse } \\
\text { scored) }\end{array}$ & & $\begin{array}{l}\text { Soft Drinks } \\
(1-100) \\
\text { (reverse scored) }\end{array}$ & $\begin{array}{l}\text { Sugar } \\
\text { sweetened } \\
\text { beverages } \\
(0-10) \\
\text { (reverse } \\
\text { scored) }\end{array}$ \\
\hline
\end{tabular}


Table 2. Summary of dietary patterns identified using factor or principal component analysis

\begin{tabular}{|c|c|}
\hline Study & Dietary Patterns \\
\hline \multirow[t]{4}{*}{ He, 2015} & $\begin{array}{l}\text { Vegetable pattern: Frequent intake of root vegetables, beans, mushrooms, melon vegetables, } \\
\text { seaweed, other legumes, fruits, leafy and cruciferous vegetables, processed vegetables, nuts, and } \\
\text { cooking oil }\end{array}$ \\
\hline & $\begin{array}{l}\text { Protein-rich pattern: Frequent intake of poultry, red meat, animal organ meat, grains (mainly } \\
\text { refined), processed meat, fish, soups, leafy and cruciferous vegetables, and eggs }\end{array}$ \\
\hline & $\begin{array}{l}\text { Prudent pattern: Frequent intake of dairy products, nuts, eggs, fish, soups, fruits; infrequent intake } \\
\text { of processed meat, sugar-sweetened beverages, and processed vegetables }\end{array}$ \\
\hline & $\begin{array}{l}\text { Sweets and seafood pattern: Cantonese desserts, mollusks and shellfish, and sugar-sweetened } \\
\text { beverages; low intakes of grains (mainly refined) and leafy and cruciferous vegetables }\end{array}$ \\
\hline \multirow[t]{2}{*}{ Radesky, 2008} & Prudent pattern: High in veg, fruit, legumes, fish, poultry, eggs, salad dressing and whole grains \\
\hline & $\begin{array}{l}\text { Western pattern: Red and processed meats, sugar-sweetened beverages, French fries, high-fat } \\
\text { dairy products, desserts, butter and refined grains }\end{array}$ \\
\hline \multirow[t]{4}{*}{ Schoenaker, 2015} & $\begin{array}{l}\text { Meats, snacks and sweets pattern: high consumption of red and processed meat, cakes, sweet } \\
\text { biscuits, fruit juice, chocolate, and pizza }\end{array}$ \\
\hline & $\begin{array}{l}\text { Mediterranean-style pattern: high factor loadings for vegetables, legumes, nuts, tofu, rice, pasta, } \\
\text { rye bread, red wine, and fish }\end{array}$ \\
\hline & $\begin{array}{l}\text { Fruit and low-fat dairy pattern: positively correlated with fruits and lowfat dairy including yoghurt, } \\
\text { low-fat cheese, and skimmed milk }\end{array}$ \\
\hline & $\begin{array}{l}\text { Cooked vegetables pattern: high consumption of carrots, peas, cooked potatoes, cauliflower, and } \\
\text { pumpkin }\end{array}$ \\
\hline Tryggvadottir, 2016 & $\begin{array}{l}\text { Prudent pattern: Positive for seafood, eggs, vegetables, fruit and berries, vegetable oils, nuts and } \\
\text { seeds, pasta, breakfast cereals, and coffee and tea; Negative for soft drinks and French fries }\end{array}$ \\
\hline \multirow[t]{2}{*}{ Zhang, 2006} & Prudent pattern: High intake of fruit, green leafy veg, poultry and fish \\
\hline & $\begin{array}{l}\text { Western pattern: High intake of red meat, processed meat, refined grain products, sweets, French } \\
\text { fries and pizza }\end{array}$ \\
\hline
\end{tabular}




\section{Table 3. Time point of exposure assessment}

Index/Score

Karamanos, 2014 (39)

Rifas-Shiman, 2009 (32)

Schoenaker, $2016(33)$

Tobias, $2012(42)$

Tryggva dottir, 2016 (43)

Zhang, 2014 (45)

PCA / Factor Analysis

He, 2015 (38)

Radesky, 2008 (40)

Schoenaker, 2015 (41)

Zhang, 2006 (44)

Other Method

Clapp, 1998 (37)

Preconception

1-6 mo preconception

First trimester

Second trimester

Dietary assessment represents the period prior to performing OGTT (24-32 wk). However, the time point of measurement or the duration are unspecified.

$<$

3,6 , or 9 yrs preconception $\leftarrow$ Cumulative avg $(2,6,10 y$ Cumulative avg $(2,6$ or $10 y$ . 


\section{Outcomes:}

Studies assessed several outcomes related to GDM, including blood glucose levels, glucose tolerance, insulin, HOMA-IR, and gestational diabetes. Table 4. Summary of outcome definitions summarizes the outcomes and diagnostic criteria grouped by methodology used to create dietary patterns.

Table 4. Summary of outcome definitions

\begin{tabular}{|c|c|c|c|c|c|c|}
\hline Study & Outcome & Diagnostic Criteria & $\begin{array}{l}\text { Source of } \\
\text { Criteria }\end{array}$ & $\begin{array}{l}\text { Method of } \\
\text { Assessment }\end{array}$ & References & $\begin{array}{l}\text { Additional } \\
\text { Outcomes } \\
\text { Measured }\end{array}$ \\
\hline \multicolumn{7}{|l|}{ Index/Score } \\
\hline $\begin{array}{l}\text { Karamanos, } \\
2014\end{array}$ & $\begin{array}{l}\text { Plasma } \\
\text { glucose levels } \\
\text { Glucose } \\
\text { tolerance } \\
\text { GDM }\end{array}$ & $\begin{array}{l}\text { GDM (ADA): } \\
\text { Requires two or more cutoff } \\
\text { points to be met or } \\
\text { exceeded; } \\
\text { Cutoff points: fasting } \geq 5.3 \text {, } \\
1-\mathrm{h} \geq 10.0 \text { and } 2-\mathrm{h} \geq 8.6 \\
\mathrm{~mm} / \mathrm{l} \\
\text { GDM (IADPSG): } \\
\text { Requires one glucose } \\
\text { value equal to or above any } \\
\text { cutoff point; } \\
\text { Cutoff points: fasting } \geq 5.1 \text {, } \\
1 \mathrm{~h} \geq 10.0, \text { and } 2 \mathrm{~h} \geq 8.5 \\
\text { mm/l }\end{array}$ & $\begin{array}{l}\text { American } \\
\text { Diabetes } \\
\text { Association } \\
\text { (ADA) } 2010 \\
\text { guidelines } \\
\text { International } \\
\text { Association of } \\
\text { the Diabetes } \\
\text { and } \\
\text { Pregnancy } \\
\text { Study Groups } \\
\text { (IADPSG) }\end{array}$ & $75 \mathrm{~g}$ OGTT & $\begin{array}{l}\text { ADA, } \\
2010^{14} \\
\text { International } \\
\text { Association } \\
\text { of Diabetes } \\
\text { and } \\
\text { Pregnancy } \\
\text { Study } \\
\text { Groups } \\
\text { Consensus } \\
\text { Panel, } \\
2010^{15}\end{array}$ & $\mathrm{~N} / \mathrm{A}$ \\
\hline
\end{tabular}

${ }^{14}$ American Diabetes, A. (2010). Diagnosis and Classification of Diabetes Mellitus. Diabetes Care, 33(Suppl 1), S62-S69. doi:10.2337/dc10-S062

${ }^{15}$ No citation provided. 


\begin{tabular}{|c|c|c|c|c|c|c|}
\hline Study & Outcome & Diagnostic Criteria & $\begin{array}{l}\text { Source of } \\
\text { Criteria }\end{array}$ & $\begin{array}{l}\text { Method of } \\
\text { Assessment }\end{array}$ & References & $\begin{array}{l}\text { Additional } \\
\text { Outcomes } \\
\text { Measured }\end{array}$ \\
\hline $\begin{array}{l}\text { Rifas-Shiman, } \\
2009\end{array}$ & $\begin{array}{l}\text { Blood glucose } \\
\text { levels } \\
\text { Impaired } \\
\text { glucose } \\
\text { tolerance } \\
\text { GDM }\end{array}$ & $\begin{array}{l}\text { If } 1 \text {-hour blood glucose } \\
\text { level was } \geq 140 \text {, referred to } \\
100 \mathrm{~g} \text { fasting glucose } 3 \text { - } \\
\text { hour tolerance test } \\
\text { Impaired glucose tolerance: } \\
\text { Failed challenge test, but } \\
\text { had } 0 \text { or } 1 \text { abnormal results } \\
\text { on fasting glucose } \\
\text { tolerance test } \\
\text { GDM: At least } 2 \text { abnormal } \\
\text { results to the } 100 \mathrm{~g} \mathrm{OGTT:} \\
\geq 95 \mathrm{mg} / \mathrm{dL} \text { ( } 5.2 \mathrm{mmol} / \mathrm{L}) \text { at } \\
\text { baseline, } \geq 180 \mathrm{mg} / \mathrm{dL}(9.9 \\
\mathrm{mmol} / \mathrm{L}) \text { at } 1 \mathrm{hour}, \geq 155 \\
\mathrm{mg} / \mathrm{dL}(8.5 \mathrm{mmol} / \mathrm{L}) \text { at } 2 \\
\text { hours, and } \geq 140 \mathrm{mg} / \mathrm{dL}(7.7 \\
\mathrm{mmol} / \mathrm{L}) \text { at } 3 \text { hours }\end{array}$ & $\begin{array}{l}\text { Carpenter- } \\
\text { Coustan (CC) } \\
\text { criteria }\end{array}$ & $\begin{array}{l}\text { Non-fasting oral } \\
\text { glucose challenge, } \\
50 \mathrm{~g}, \text { followed by } \\
100 \mathrm{~g} \text { OGTT if } \\
\text { needed }\end{array}$ & $\begin{array}{l}\text { Carpenter, } \\
1982^{16}\end{array}$ & $\begin{array}{l}\text { Preeclampsia } \\
\text { Gestational } \\
\text { weight gain } \\
\text { Birth weight }\end{array}$ \\
\hline $\begin{array}{l}\text { Schoenaker, } \\
2016\end{array}$ & GDM & $\begin{array}{l}\text { Glucose intolerance with } \\
\text { onset or first recognition } \\
\text { during pregnancy. Fasting } \\
\text { glucose } \geq 5.5 \mathrm{mmol} / \mathrm{dL} \\
\text { and/or } 2-\mathrm{h} \text { level of } \geq 8.0 \\
\mathrm{mmol} / \mathrm{L} \text { at } 24-28 \mathrm{wk} \text { of } \\
\text { gestation }\end{array}$ & $\begin{array}{l}\text { The } \\
\text { Australasian } \\
\text { Diabetes in } \\
\text { Pregnancy } \\
\text { Society }\end{array}$ & $\begin{array}{l}\text { Self-reported } \\
\text { based on } \\
\text { physician } \\
\text { diagnosis between } \\
2003 \text { and } 2012\end{array}$ & $\begin{array}{l}\text { Hoffman, } \\
1998^{17}\end{array}$ & $\begin{array}{l}\text { Hypertensive } \\
\text { disorders of } \\
\text { pregnancy }\end{array}$ \\
\hline
\end{tabular}

${ }^{16}$ Carpenter, M. W., \& Coustan, D. R. (1982). Criteria for screening tests for gestational diabetes. Am J Obstet Gynecol, 144(7), 768-773.

${ }^{17}$ Hoffman, L., Nolan, C., Wilson, J. D., Oats, J. J., \& Simmons, D. (1998). Gestational diabetes mellitus--management guidelines. The Australasian Diabetes in Pregnancy Society. Med J Aust, 169(2), 93-97. 


\begin{tabular}{|c|c|c|c|c|c|c|}
\hline Study & Outcome & Diagnostic Criteria & $\begin{array}{l}\text { Source of } \\
\text { Criteria }\end{array}$ & $\begin{array}{l}\text { Method of } \\
\text { Assessment }\end{array}$ & References & $\begin{array}{l}\text { Additional } \\
\text { Outcomes } \\
\text { Measured }\end{array}$ \\
\hline Tobias, 2012 & GDM & $\begin{array}{l}\text { Requires at least two } \\
\text { values at or above the } \\
\text { following cutoff values: } \\
\text { fasting, } 1 \text {-hour, 2-hour, and } \\
\text { 3-hour plasma glucose } \\
\text { levels of } \geq 105 \mathrm{mg} / \mathrm{dL} \text {, } \\
\geq 190 \mathrm{mg} / \mathrm{dL}, \geq 165 \mathrm{mg} / \mathrm{dL}, \\
\text { and } \geq 145 \mathrm{mg} / \mathrm{dL} \\
\text { respectively }\end{array}$ & $\begin{array}{l}\text { National } \\
\text { Diabetes } \\
\text { Data Group } \\
\text { (most but not } \\
\text { all physicians } \\
\text { reportedly } \\
\text { used these } \\
\text { criteria) }\end{array}$ & $\begin{array}{l}\text { Self-reported } \\
\text { physician } \\
\text { diagnosis }\end{array}$ & $\begin{array}{l}\text { NDDG, } \\
1979^{18}\end{array}$ & $\mathrm{~N} / \mathrm{A}$ \\
\hline $\begin{array}{l}\text { Tryggvadottir, } \\
2016\end{array}$ & $\begin{array}{l}\text { Serum } \\
\text { glucose } \\
\text { Insulin } \\
\text { HOMA-IR } \\
\text { GDM }\end{array}$ & $\begin{array}{l}\text { GDM: One or more } \\
\text { abnormal values from the } \\
\text { following: } \\
\text { Fasting plasma glucose } \\
\text { between } 5.1 \text { and } 6.9 \\
\mathrm{mmol} / \mathrm{l}, 1-\mathrm{h} \text { value of } \geq 10.0 \\
\mathrm{mmol} / \mathrm{l} \text { or a } 2 \text {-h plasma } \\
\text { glucose of } 8.5-11.0 \mathrm{mmol} / /\end{array}$ & WHO & 2h, $75 \mathrm{~g} \mathrm{OGTT}$ & $\begin{array}{l}\text { WHO, } \\
2013^{19}\end{array}$ & $\mathrm{~N} / \mathrm{A}$ \\
\hline
\end{tabular}

${ }^{18}$ Classification and diagnosis of diabetes mellitus and other categories of glucose intolerance. National Diabetes Data Group. (1979). Diabetes, 28(12), $1039-1057$.

${ }^{19}$ Diagnostic Criteria and Classification of Hyperglycaemia First Detected in Pregnancy. (2013). Geneva: World Health Organization. 


\begin{tabular}{|c|c|c|c|c|c|c|}
\hline Study & Outcome & Diagnostic Criteria & $\begin{array}{l}\text { Source of } \\
\text { Criteria }\end{array}$ & $\begin{array}{l}\text { Method of } \\
\text { Assessment }\end{array}$ & References & $\begin{array}{l}\text { Additional } \\
\text { Outcomes } \\
\text { Measured }\end{array}$ \\
\hline Zhang, 2014 & GDM & $\begin{array}{l}\text { Requires at least two } \\
\text { values at or above the } \\
\text { following cutoff values: } \\
\text { fasting, } 1 \text {-hour, } 2 \text {-hour, and } \\
\text { 3-hour plasma glucose } \\
\text { levels of } \geq 105 \mathrm{mg} / \mathrm{dL} \text {, } \\
\geq 190 \mathrm{mg} / \mathrm{dL}, \geq 165 \mathrm{mg} / \mathrm{dL} \text {, } \\
\text { and } \geq 145 \mathrm{mg} / \mathrm{dL} \text {, } \\
\text { respectively }\end{array}$ & $\begin{array}{l}\text { National } \\
\text { Diabetes } \\
\text { Data Group } \\
\text { (most but not } \\
\text { all physicians } \\
\text { reportedly } \\
\text { used these } \\
\text { criteria) }\end{array}$ & $\begin{array}{l}\text { Self-reported } \\
\text { physician } \\
\text { diagnosis }\end{array}$ & $\begin{array}{l}\text { NDDG, } \\
1979^{20}\end{array}$ & $\mathrm{~N} / \mathrm{A}$ \\
\hline \multicolumn{7}{|l|}{$\begin{array}{l}\text { PCA/Factor } \\
\text { Analysis }\end{array}$} \\
\hline He, 2015 & GDM & $\begin{array}{l}\text { Blood glucose values met } \\
\text { or exceeded the following } \\
\text { criteria: fasting, } 5.1 \mathrm{mmol} / \mathrm{L} \text {; } \\
\text { 1h, } 10.0 \mathrm{mmol} / \mathrm{L} ; 2 \mathrm{~h}, 8.5 \\
\mathrm{mmol} / \mathrm{L}\end{array}$ & $\begin{array}{l}\text { International } \\
\text { Association of } \\
\text { Diabetes and } \\
\text { Pregnancy } \\
\text { Study Groups } \\
\text { (IADPSG) }\end{array}$ & $75 \mathrm{~g}, 2 \mathrm{~h}$ OGTT & $\begin{array}{l}\text { Metzger, } \\
2010^{21}\end{array}$ & $\mathrm{~N} / \mathrm{A}$ \\
\hline
\end{tabular}

${ }^{20}$ Classification and diagnosis of diabetes mellitus and other categories of glucose intolerance. National Diabetes Data Group. (1979). Diabetes, 28(12), $1039-1057$.

21 International Association of Diabetes \& Pregnancy Study Groups Consensus Panel. (2010). International Association of Diabetes and Pregnancy Study Groups

Recommendations on the Diagnosis and Classification of Hyperglycemia in Pregnancy. Diabetes Care, 33(3), 676-682. doi:10.2337/dc09-1848 


\begin{tabular}{|c|c|c|c|c|c|c|}
\hline Study & Outcome & Diagnostic Criteria & $\begin{array}{l}\text { Source of } \\
\text { Criteria }\end{array}$ & $\begin{array}{l}\text { Method of } \\
\text { Assessment }\end{array}$ & References & $\begin{array}{l}\text { Additional } \\
\text { Outcomes } \\
\text { Measured }\end{array}$ \\
\hline $\begin{array}{l}\text { Radesky, } \\
2008\end{array}$ & $\begin{array}{l}\text { Impaired } \\
\text { glucose } \\
\text { tolerance } \\
\text { GDM }\end{array}$ & 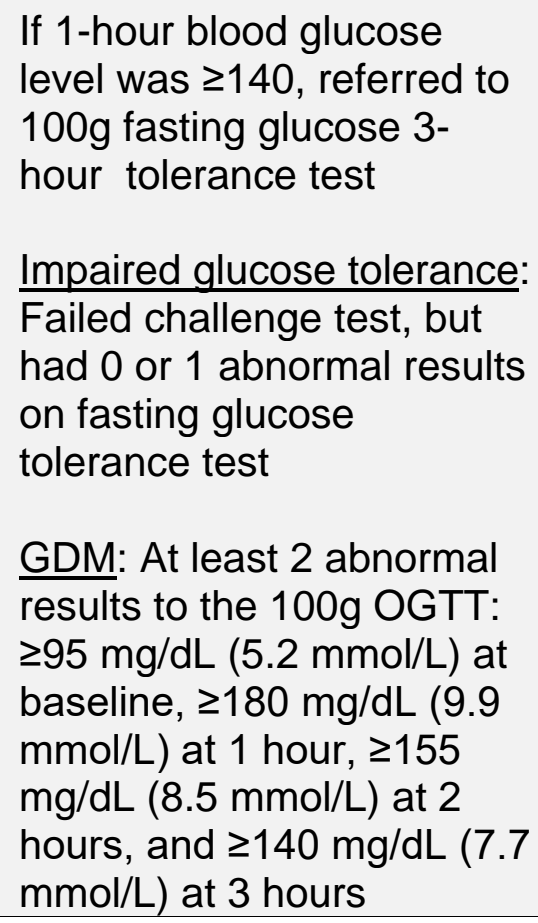 & $\begin{array}{l}\text { Carpenter- } \\
\text { Coustan (CC) } \\
\text { criteria }\end{array}$ & $\begin{array}{l}\text { Non-fasting oral } \\
\text { glucose challenge, } \\
50 \mathrm{~g}, \text { followed by } \\
100 \mathrm{~g} \text { OGTT if } \\
\text { needed }\end{array}$ & $\begin{array}{l}\text { Carpenter, } \\
1982^{22}\end{array}$ & $\mathrm{~N} / \mathrm{A}$ \\
\hline
\end{tabular}

${ }^{22}$ Carpenter, M. W., \& Coustan, D. R. (1982). Criteria for screening tests for gestational diabetes. Am J Obstet Gynecol, 144(7), 768-773. 


\begin{tabular}{|c|c|c|c|c|c|c|}
\hline Study & Outcome & Diagnostic Criteria & $\begin{array}{l}\text { Source of } \\
\text { Criteria }\end{array}$ & $\begin{array}{l}\text { Method of } \\
\text { Assessment }\end{array}$ & References & $\begin{array}{l}\text { Additional } \\
\text { Outcomes } \\
\text { Measured }\end{array}$ \\
\hline $\begin{array}{l}\text { Schoenaker, } \\
2015\end{array}$ & GDM & $\begin{array}{l}\text { During study period, } \\
\text { diagnostic criteria for GDM } \\
\text { in Australia included } 1 \mathrm{~h} \\
\text { venous plasma glucose } \\
\text { level } \geq 6.55 \% \text { ( } 7.8 \mathrm{mmol} / \mathrm{l}) \\
\text { after } 50 \mathrm{~g} \text { glucose load, or } \\
1 \mathrm{~h} \text { venous plasma glucose } \\
\text { level } \geq 6.65 \% \text { ( } 8.0 \mathrm{mmol} / \mathrm{l}) \\
\text { after a } 75 \mathrm{~g} \text { glucose load; } \\
\text { diagnosis confirmed with } \\
75 \mathrm{~g} \text { OGTT (fasting) with a } \\
\text { venous plasma glucose } \\
\text { level at } 0 \mathrm{~h} \text { of } \geq 5.1 \% \text { ( } 5.6 \\
\mathrm{mmol} / \mathrm{l}) \text { and/or at } 2 \mathrm{~h} \text { of } \\
\geq 6.65 \% \text { ( } 8.0 \mathrm{mmol} / \mathrm{l})\end{array}$ & $\begin{array}{l}\text { The } \\
\text { Australasian } \\
\text { Diabetes in } \\
\text { Pregnancy } \\
\text { Society }\end{array}$ & $\begin{array}{l}\text { Self-reported } \\
\text { physician } \\
\text { diagnosis in } \\
\text { response to } \\
\text { question, "Were } \\
\text { you diagnosed or } \\
\text { treated for } \\
\text { gestational } \\
\text { diabetes?" }\end{array}$ & $\begin{array}{l}\text { Hoffman, } \\
1998^{23}\end{array}$ & $\mathrm{~N} / \mathrm{A}$ \\
\hline Zhang, 2006 & GDM & $\begin{array}{l}\text { Requires at least two } \\
\text { values at or above the } \\
\text { following cutoff values: } \\
\text { fasting, } 1 \text {-hour, } 2 \text {-hour, and } \\
\text { 3-hour plasma glucose } \\
\text { levels of } \geq 105 \mathrm{mg} / \mathrm{dL} \text {, } \\
\geq 190 \mathrm{mg} / \mathrm{dL}, \geq 165 \mathrm{mg} / \mathrm{dL} \text {, } \\
\text { and } \geq 145 \mathrm{mg} / \mathrm{dL} \text {, } \\
\text { respectively }\end{array}$ & $\begin{array}{l}\text { National } \\
\text { Diabetes } \\
\text { Data Group } \\
\text { (most but not } \\
\text { all physicians } \\
\text { reportedly } \\
\text { used these } \\
\text { criteria) }\end{array}$ & $\begin{array}{l}\text { Self-reported } \\
\text { physician } \\
\text { diagnosis }\end{array}$ & $\begin{array}{l}\text { NDDG, } \\
1979^{24}\end{array}$ & $\mathrm{~N} / \mathrm{A}$ \\
\hline Other Metho & & & & & & \\
\hline
\end{tabular}

${ }^{23}$ Hoffman, L., Nolan, C., Wilson, J. D., Oats, J. J., \& Simmons, D. (1998). Gestational diabetes mellitus--management guidelines. The Australasian Diabetes in Pregnancy
Society. Med J Aust, 169(2), 93-97.
${ }^{24}$ Classification and diagnosis of diabetes mellitus and other categories of glucose intolerance. National Diabetes Data Group. (1979). Diabetes, 28(12), 1039-1057. 


\begin{tabular}{|c|c|c|c|c|c|c|}
\hline Study & Outcome & Diagnostic Criteria & $\begin{array}{l}\text { Source of } \\
\text { Criteria }\end{array}$ & $\begin{array}{l}\text { Method of } \\
\text { Assessment }\end{array}$ & References & $\begin{array}{l}\text { Additional } \\
\text { Outcomes } \\
\text { Measured }\end{array}$ \\
\hline Clapp, 1998 & $\begin{array}{l}\text { Blood glucose } \\
\text { Serum insulin }\end{array}$ & $\mathrm{N} / \mathrm{A}$ & $\mathrm{N} / \mathrm{A}$ & $\begin{array}{l}\text { Blood glucose: } \\
\text { glucose oxidase } \\
\text { methodology and } \\
\text { an accurately } \\
\text { calibrated Yellow } \\
\text { Springs glucose } \\
\text { and lactate } \\
\text { analyzer } \\
\text { Serum insulin: } \\
\text { double antibody } \\
\text { radioimmunoassay }\end{array}$ & $\mathrm{N} / \mathrm{A}$ & $\mathrm{N} / \mathrm{A}$ \\
\hline
\end{tabular}




\section{Evidence synthesis}

With 10 prospective cohort studies and $1 \mathrm{RCT}$, there is a modest body of evidence available to examine the relationship between dietary patterns before and during pregnancy and risk of GDM. Table 5. Results grouped by methodology used for dietary pattern assessment, ${ }_{2}$ provides more information on the findings of each of these studies. There is substantial heterogeneity in the methodology employed to define and assess dietary patterns, which makes it difficult to compare across studies. Also, the time point of dietary assessment is variable. Despite these differences, there is modest evidence of an association between dietary patterns before pregnancy and risk of GDM.

\section{Dietary patterns assessed via index/score}

Six studies used indices/scores to assess dietary patterns. Three of these found an inverse association between greater adherence to a healthy diet before and during pregnancy and risk of GDM and one study found that lower adherence to a healthy diet is associated with greater GDM risk $(3,7,8,11)$.

Before Pregnancy: Studies that assessed maternal diet before pregnancy using an index/score method indicated that diets rich in vegetables, legumes, fruits and nuts, whole grains, and low in meat (specifically red and processed meats), sugar sweetened beverages and trans fat were associated with lower risk of GDM. A summary of findings across studies is presented below.

- Schoenaker et al.(7) assessed adherence to a Mediterranean dietary pattern before pregnancy. Beneficial dietary components included vegetables, legumes, fruits and nuts, cereals, fish and monounsaturated fat:saturated fat ratio, and detrimental dietary components included meat, poultry and high-fat dairy. Lower adherence to the Mediterranean diet, in comparison to higher adherence, was associated with increased odds of GDM.

- Both Tobias et al.(8) and Zhang et al. (11) used data from the NHS II and assessed the association between diet before pregnancy and risk of GDM.

○ Zhang et al. (11) looked at the Alternate Healthy Eating Index-2010, which is characterized by higher intake of vegetables, fruit, nuts, whole grains, polyunsaturated fatty acids and long-chain omega- 3 fatty acids and lower intake of red and processed meats, sugar sweetened beverages, trans fat and sodium. The authors concluded that higher adherence to the modified Alternate Healthy Eating Index-2010 was associated with a decreased risk of GDM.

- Tobias et al. (8) assessed dietary adherence to three different dietary patterns: alternate Mediterranean Diet, Dietary Approaches to Stop Hypertension (DASH), and alternate Healthy Eating Index (aHEI).

- Alternate Mediterranean diet: Positively scored components were fruits, vegetables, nuts, legumes, soy, fish and seafood, whole grains, moderate alcohol, MUFA:SFA, and the negatively-scored components were red and processed meat

- Dietary Approaches to Stop Hypertension (DASH): Positivelyscored components were fruits, vegetables, nuts, legumes, soy, whole grains, low-fat dairy, and the negatively-scored components were red and processed meats, sweetened beverages and 
sodium

- Alternative Healthy Eating Index (aHEI): Positively-scored components were fruits, vegetables, nuts, legumes, soy, white:red meat ratio, cereal fiber, moderate alcohol, PUFA:SFA, multivitamin use, and the negatively-scored component included trans fat

In this study, higher adherence to a healthy dietary pattern was inversely associated with the risk of GDM, irrespective of the approach used to assess dietary patterns.

Pregnancy: Results were heterogeneous when examining dietary patterns during pregnancy and risk of GDM. Rifas-Shiman et al.(5) showed that adherence to the Alternate Healthy Eating Index improved blood glucose level but was not associated with GDM. Similarly, Tryggvadottir et al.(9) reported a significant association only for those who were overweight and those with obesity. Karamanos et al.(3) showed that a Mediterranean dietary pattern was associated with reduced risk of GDM, but it is unclear at which point during pregnancy diet was measured. Tryggvadottir et al. (9) collected dietary data right before GDM assessment (19 to 24 weeks) with a relatively short recall period of 4 days. Rifas-Shiman et al.(5) assessed dietary data at the end of the first trimester (11.7 weeks), and the recall period was the entire first trimester. A summary of findings across studies is presented below.

- In a multi-country study, Karamanos et al. (3)assessed the association between Mediterranean diet intake during pregnancy and the incidence of GDM. The Mediterranean diet was characterized by intake of bread, cereals, legumes, vegetables, fruits, meat, fish, eggs, the ratio of olive oil to animal fat, potatoes, cheese, and dairy products. Some of the "healthy" foods included vegetables and legumes and the "unhealthy" foods included meat and eggs. The study found that greater adherence to a Mediterranean dietary pattern was inversely associated with the risk of GDM and improved glucose tolerance.

- Tryggvadottir et al.(9) used a Healthy Eating Index that assessed conformance to food-based dietary guidelines from the Icelandic Directorate of Health. The scores were based on intake of fish and seafood, vegetables, fruits, vegetable oils, nuts and seeds, unground/wholeground cereals, soft drinks, and vitamin D. The results from an unadjusted analysis showed that higher adherence to a healthy diet was correlated with a lower incidence of GDM, but this was observed only among overweight women and those with obesity.

- Rifas-Shiman et al. (5) used an Alternate Healthy Eating Index (modified for pregnancy), which was characterized by intake of vegetables, fruit, ratio of white to red meat, fiber, trans fat, ratio of polyunsaturated to saturated fatty acids, and folate, calcium, and iron from foods. The study showed that while adherence to this dietary pattern was associated with improved blood glucose level, there was no association with GDM.

\section{Dietary patterns assessed via factor or principal component analysis}

Five studies used data-driven methods (i.e., principal component analysis or exploratory factor analysis) to assess dietary patterns. These studies found an inverse association between greater adherence to vegetable, Mediterranean-style and prudent dietary patterns and risk of $\operatorname{GDM}(2,6,9,10)$. Also, studies found that higher 
adherence to a sweets and seafood dietary pattern and Western pattern were associated with the increased risk of GDM.

Before Pregnancy: Studies that assessed maternal diet before pregnancy indicated that a diet rich in vegetables, legumes, and nuts and low in processed meat was inversely associated with the risk of GDM.

- Schoenaker et al. (6) demonstrated that higher adherence to a Mediterranean style dietary pattern (characterized by high factor loadings for vegetables, legumes, nuts, tofu, rice, pasta, rye bread, red wine, and fish) before pregnancy was associated with a reduced risk of GDM. The study also assessed other patterns such as 1) meat, snacks and sweet pattern (characterized by high consumption of red and processed meat, cakes, sweet biscuits, fruit juice, chocolate, and pizza), 2) fruit and low-fat dairy pattern (positively correlated with fruits and low fat dairy including yogurt, low-fat cheese, and skimmed milk) and 3 ) cooked vegetables pattern (characterized by high consumption of carrots, peas, cooked potatoes, cauliflower, and pumpkin). These patterns were not associated with the risk of GDM.

- Zhang et al. (10) identified two dietary patterns: 1) Prudent dietary pattern with high intake of components such as fruits, green leafy vegetables, poultry and fish; 2) Western dietary pattern with higher intake of components such as red meat, processed meat, refined grain products, sweets, French fries and pizza. Greater adherence to a Western dietary pattern and lower adherence to a prudent dietary pattern before pregnancy was associated with an increased risk of GDM.

Pregnancy: Results of the studies that assessed dietary patterns during pregnancy and risk of GDM were mixed. For example, He et al.(2) and Tryggvadottir et al. (9) demonstrated that greater adherence to a vegetable dietary pattern and a prudent dietary pattern was associated with reduced GDM risk, while greater adherence to a sweets and seafood pattern was associated with an increased GDM risk. In both studies, diet was assessed only in second trimester (between 19 to 27 weeks) and the recall period was relatively short (4 days in Tryggvadottir et al.(9) and 1 week in He et al. (2) study). Radesky et al.(4) showed no association between prudent western dietary patterns and GDM risk. In this study, dietary data were collected around 11.8 weeks, and the recall period was from the time of conception until data collection. A summary of findings across studies is presented below.

- He et al. (2) demonstrated that higher adherence to a vegetable dietary pattern (characterized by positive loadings for root vegetables, beans, mushrooms, melon vegetables, seaweed, other legumes, fruits, leafy and cruciferous vegetables, processed vegetables, nuts and cooking oil) was inversely associated with the risk of GDM. The same study also showed that higher adherence to sweets and seafood pattern (characterized by positive loadings for Cantonese desserts, molluscs and shellfish, sugar-sweetened beverages, grains and leafy and cruciferous vegetables) were associated with an increased risk of GDM.

- Tryggvadottir et al. (9) demonstrated that a prudent dietary pattern (characterized by positive loadings for seafood, eggs, vegetables, fruit and berries, vegetable oils, nuts and seeds, pasta, breakfast cereals, and coffee and tea, and negative for soft drinks and French fries) was associated with a lower 
risk of GDM.

- Radesky et al. (4) identified two dietary patterns: 1) prudent dietary pattern characterized by high intake of vegetables, fruit, legumes, fish, poultry, eggs, salad dressing and whole grains; and 2) Western dietary pattern characterized by high intake of red and processed meats, sugar-sweetened beverages, French fries, high fat dairy products, desserts, butters and refined grains. Neither of these patterns was associated with the risk of impaired glucose tolerance or GDM.

\section{Dietary patterns assessed using other methods}

This body of evidence includes one pilot RCT with 12 subjects. At 8 weeks of gestation, subjects were randomized to an aboriginal carbohydrate diet (a low glycemic index diet characterized by carbohydrates from unprocessed whole grains, fruits, beans, vegetables, and many dairy products; includes most dense whole grain and multigrain breads, bran cereals, pastas, fresh fruits and vegetables, yogurt, ice cream, and nuts) or a cafeteria carbohydrate diet (a high glycemic index diet characterized by carbohydrates from highly processed grains, root vegetables, and simple sugars; includes many highly refined breads, potatoes, instant rice, most breakfast cereals, desserts, and snack-type foods). Randomization to a cafeteria diet was associated with an increase in glucose response in mid- and late-pregnancy. Note that the study did not assess GDM. 
Table 5. Results grouped by methodology used for dietary pattern assessment

\begin{tabular}{|l|}
\hline Key for color-coding: \\
\hline Dietary pattern categorized as beneficial when... \\
Greater adherence reduces risk of GDM \\
Lower adherence increases risk of GDM \\
\hline Dietary pattern categorized as detrimental when... \\
Greater adherence increases risk of GDM \\
Lower adherence decreases risk of GDM \\
\hline
\end{tabular}

\begin{tabular}{|c|c|c|c|c|}
\hline $\begin{array}{l}\text { Author, Year } \\
\text { Country, N }\end{array}$ & Exposure & Outcome & Significant finding & NS finding \\
\hline \multicolumn{5}{|l|}{ Index/Score } \\
\hline \multicolumn{5}{|l|}{ Before Pregnancy } \\
\hline Schoenaker, 2016 & Mediterranean Diet Score & GDM (OR) & \multirow{5}{*}{$\begin{array}{l}\text { Total effect (per } 1- \\
\mathrm{kg} / \mathrm{m} 2 \text { increase in } \\
\mathrm{BMI}): \\
1.35 \\
(95 \% \mathrm{Cl}: 1.02,1.60)\end{array}$} & \\
\hline \multirow{4}{*}{$\begin{array}{l}\text { Australia } \\
\mathrm{N}=3,378\end{array}$} & \multirow{2}{*}{$\begin{array}{l}\text { For beneficial foods (vegetables, } \\
\text { legumes, fruit and nuts, cereals, fish, } \\
\text { and mono-:saturated fat ratio), a value } \\
\text { of } 1 \text { was given if above the median, } 0 \text { if } \\
\text { below }\end{array}$} & $\mathrm{High}=\mathrm{REF}$ & & \\
\hline & & & & \\
\hline & $\begin{array}{l}\text { For detrimental foods (meat, poultry, } \\
\text { high-fat dairy), a value of } 0 \text { was given if } \\
\text { above the median, } 1 \text { if below }\end{array}$ & & & \\
\hline & $\begin{array}{l}\text { For alcohol, a value of } 1 \text { was given for } \\
\text { daily consumption of } 5-15 \mathrm{~g} / \mathrm{d}\end{array}$ & & & \\
\hline
\end{tabular}




\begin{tabular}{|c|c|c|c|c|}
\hline $\begin{array}{l}\text { Author, Year } \\
\text { Country, N }\end{array}$ & Exposure & Outcome & Significant finding & NS finding \\
\hline \multirow{16}{*}{$\begin{array}{l}\text { Tobias, } 2012 \\
\text { USA } \\
N=21,376 \text { births } \\
\text { (15,254 } \\
\text { participants) }\end{array}$} & Alternate Mediterranean (aMED) & GDM (RR) & \multirow{5}{*}{$\begin{array}{l}\text { Q3: } 0.76 \\
(95 \% \mathrm{Cl}: 0.60,0.95) \\
\text { Q4: } 0.76 \\
(95 \% \mathrm{Cl}: 0.60,0.95) \\
\text { For } 1 \mathrm{SD} \text { increase in } \\
\text { adherence: } 0.90 \\
(95 \% \mathrm{Cl}: 0.83,0.97)\end{array}$} & \multirow{5}{*}{$\begin{array}{l}\text { Q2: } 0.95 \\
(95 \% \mathrm{Cl}: 0.79,1.14)\end{array}$} \\
\hline & Positively-scored components: Fruit; & \multirow[t]{4}{*}{ Q1=REF } & & \\
\hline & $\begin{array}{l}\text { vegetables; nuts, legumes, soy; fish } \\
\text { and seafood; whole grains; moderate }\end{array}$ & & & \\
\hline & alcohol; MUFA:SFA & & & \\
\hline & $\begin{array}{l}\text { Negatively-scored components: Red } \\
\text { and processed meat }\end{array}$ & & & \\
\hline & Dietary Approaches to Stop & \multirow{6}{*}{$\begin{array}{l}\text { GDM (RR) } \\
\text { Q1=REF }\end{array}$} & Q2: 0.77 & \\
\hline & Hypertension (DASH) & & (95\% Cl: 0.63, 0.93) & \\
\hline & $\frac{\text { Positively-scored components: Fruit; }}{\text { vegetables; nuts, legumes, soy; whole }}$ & & $\begin{array}{l}\text { Q3: } 0.78 \\
(95 \% \mathrm{Cl}: 0.64,0.95)\end{array}$ & \\
\hline & grains; low-fat dairy & & Q4: 0.66 & \\
\hline & Negatively-scored components: Red & & (95\% Cl: 0.53, 0.82) & \\
\hline & $\begin{array}{l}\text { and processed meats, sweetened } \\
\text { beverages, sodium }\end{array}$ & & $\begin{array}{l}\text { For } 1 \text { SD increase in } \\
\text { adherence: } 0.85 \\
(95 \% \text { Cl: } 0.79,0.92)\end{array}$ & \\
\hline & Alternative Healthy Eating Index & \multirow{5}{*}{$\begin{array}{l}\text { GDM (RR) } \\
\text { Q1=REF }\end{array}$} & Q3: 0.75 & \multirow{5}{*}{$\begin{array}{l}\text { Q2: } 0.96 \\
(95 \% \mathrm{Cl}: 0.79,1.15)\end{array}$} \\
\hline & (aHEI) & & (95\% Cl: 0.61, 0.91) & \\
\hline & \multirow{2}{*}{$\begin{array}{l}\text { Positively-scored components: Fruit; } \\
\text { vegetables; nuts, legumes, soy; } \\
\text { white:red meat ratio; cereal fiber; } \\
\text { moderate alcohol; PUFA:SFA; } \\
\text { multivitamin use }\end{array}$} & & $\begin{array}{l}\text { Q4: } 0.54 \\
(95 \% \mathrm{Cl}: 0.43,0.68)\end{array}$ & \\
\hline & & & \multirow[t]{2}{*}{$\begin{array}{l}\text { For } 1 \text { SD increase in } \\
\text { adherence: } 0.76 \\
(95 \% \text { Cl: } 0.70,0.82)\end{array}$} & \\
\hline & Negatively-scored components: trans & & & \\
\hline
\end{tabular}




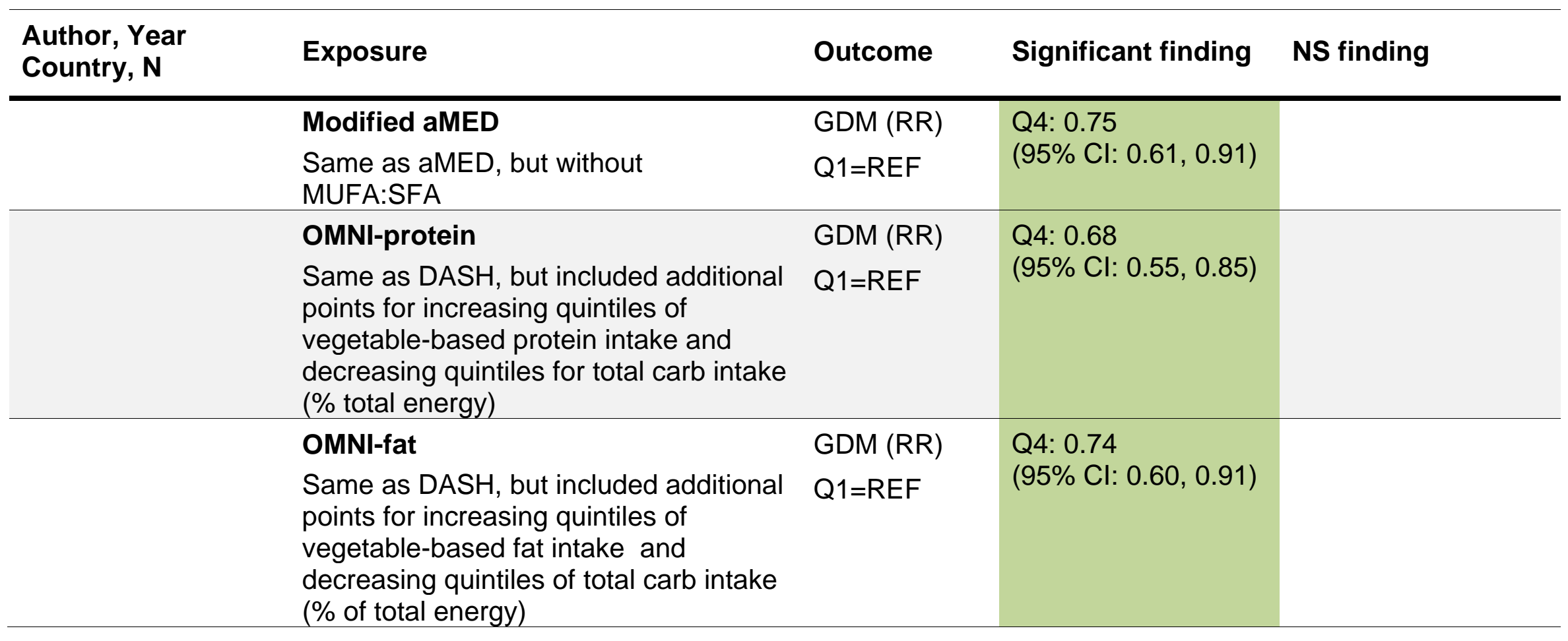




\begin{tabular}{|c|c|c|c|c|}
\hline $\begin{array}{l}\text { Author, Year } \\
\text { Country, N }\end{array}$ & Exposure & Outcome & Significant finding & NS finding \\
\hline \multirow{3}{*}{$\begin{array}{l}\text { Zhang, } 2014 \\
\text { USA } \\
N=20,136 \text { births } \\
\text { (14,437 } \\
\text { participants) }\end{array}$} & $\begin{array}{l}\text { Modified Alternative Healthy Eating } \\
\text { Index-2010 }\end{array}$ & GDM (RR) & $\begin{array}{l}\text { Q5: } 0.75 \\
(95 \% \mathrm{Cl}: 0.59,0.94)\end{array}$ & $\begin{array}{l}\text { Q2: } 0.96 \\
(95 \% \mathrm{Cl}: 0.79,1.17)\end{array}$ \\
\hline & \multirow{2}{*}{$\begin{array}{l}\text { Positively-scored components: } \\
\text { vegetables, fruit, nuts, whole grains, } \\
\text { polyunsaturated fatty acids, and long } \\
\text { chain omega } 3 \text { fatty acids }\end{array}$} & & \multirow{2}{*}{$\begin{array}{l}\text { Healthy diet (score } \\
\text { in upper two-fifths vs } \\
\text { lower three-fifths): } \\
0.81 \\
(95 \% \mathrm{Cl}: 0.70,0.94)\end{array}$} & $\begin{array}{l}\text { Q3: } 0.98 \\
(95 \% \mathrm{Cl}: 0.80,1.21)\end{array}$ \\
\hline & & & & $\begin{array}{l}\text { Q4: } 0.84 \\
(95 \% \mathrm{Cl}: 0.67,1.04)\end{array}$ \\
\hline \multirow{6}{*}{$\begin{array}{l}\text { Karamanos, } 2014 \\
\text { Algeria, France, } \\
\text { Greece, Italy, } \\
\text { Lebanon, Malta, } \\
\text { Morocco, Serbia, } \\
\text { Syria, Tunisia } \\
\text { N=1,003 }\end{array}$} & \multirow{5}{*}{$\begin{array}{l}\text { Mediterranean Diet (MedDiet) Score } \\
1 \text { point given for intake above the } \\
\text { median for "healthy foods (that is, } \\
\text { vegetables, legumes and so on)," and } 1 \\
\text { point given for intake below the median } \\
\text { for "less healthy foods (that is, meat, } \\
\text { eggs and so on)" }\end{array}$} & Glucose & \multirow{6}{*}{$\begin{array}{l}\text { Plasma glucose } 1 \mathrm{~h} \\
\text { post-load: } \\
8.0 \pm 0.1 \\
\text { (low MedDiet score) } \\
\text { vs } \\
7.7 \pm 0.1 \\
\text { (high MedDiet score) }\end{array}$} & \multirow{6}{*}{$\begin{array}{l}\text { Fasting plasma } \\
\text { glucose: } \\
4.6 \pm 0.1 \text { (low) vs } \\
4.5 \pm 0.1 \text { (high) } \\
\text { Plasma glucose } 2 \mathrm{~h} \\
\text { post-load: } \\
6.8 \pm 0.1 \text { (low) vs } \\
6.6 \pm 0.1 \text { (high) }\end{array}$} \\
\hline & & tolerance & & \\
\hline & & $\begin{array}{l}\text { (Mean } \pm \\
\text { SEM; mmol/l) }\end{array}$ & & \\
\hline & & & & \\
\hline & & & & \\
\hline & \multicolumn{2}{|l|}{$\begin{array}{l}\text { Components: bread, cereals, legumes, } \\
\text { vegetables, fruits, meat, fish, eggs, the } \\
\text { ratio of olive oil to animal fat, potatoes, } \\
\text { cheese, and dairy products }\end{array}$} & & \\
\hline
\end{tabular}




\begin{tabular}{|c|c|c|c|c|}
\hline $\begin{array}{l}\text { Author, Year } \\
\text { Country, N }\end{array}$ & Exposure & Outcome & Significant finding & NS finding \\
\hline & & $\begin{array}{l}\text { GDM (ADA; } \\
\text { OR) } \\
\text { Low=REF }\end{array}$ & $\begin{array}{l}\text { High: } 0.618 \\
(95 \% \mathrm{Cl}: 0.401 \\
0.95)\end{array}$ & \\
\hline & & $\begin{array}{l}\text { GDM } \\
\text { (IADPSG; } \\
\text { OR) }\end{array}$ & \multirow[t]{2}{*}{$\begin{array}{l}\text { High: } 0.655 \\
(95 \% \text { Cl: } 0.495 \text {, } \\
0.867)\end{array}$} & \\
\hline & & Low=REF & & \\
\hline \multirow{5}{*}{$\begin{array}{l}\text { Rifas-Shiman, } 2009 \\
\text { USA } \\
N=1,777\left(1^{\text {st }}\right. \\
\text { trimester) }\end{array}$} & \multirow{5}{*}{$\begin{array}{l}\text { Alternate Healthy Eating Index for } \\
\text { Pregnancy (AHEI-P) } \\
\text { Components: vegetables; fruit; ratio of } \\
\text { white to red meat; fiber; trans fat; ratio } \\
\text { of polyunsaturated to saturated fatty } \\
\text { acids; and folate, calcium, and iron from } \\
\text { foods }\end{array}$} & $\begin{array}{l}\text { Blood glucose } \\
\text { levels }\end{array}$ & \multirow{2}{*}{$\begin{array}{l}\text { 1st trimester: } \\
-0.64 \\
(95 \% \mathrm{Cl}:-1.25,- \\
0.02)\end{array}$} & \\
\hline & & $\begin{array}{l}\text { ( } \beta \text { for each } 5 \\
\text { point increase } \\
\text { in AHEI-P } \\
\text { score; } \mathrm{mg} / \mathrm{dL} \text { ) }\end{array}$ & & \\
\hline & & $\begin{array}{l}\text { glucose } \\
\text { tolerance }\end{array}$ & & $\begin{array}{l}1.00 \\
(95 \% \mathrm{Cl}: 0.93,1.08)\end{array}$ \\
\hline & & $\begin{array}{l}\text { (OR - for } \\
\text { each } 5 \text { point } \\
\text { increase in } \\
\text { AHEI-P } \\
\text { score) }\end{array}$ & & \\
\hline & & Normal=REF & & \\
\hline
\end{tabular}




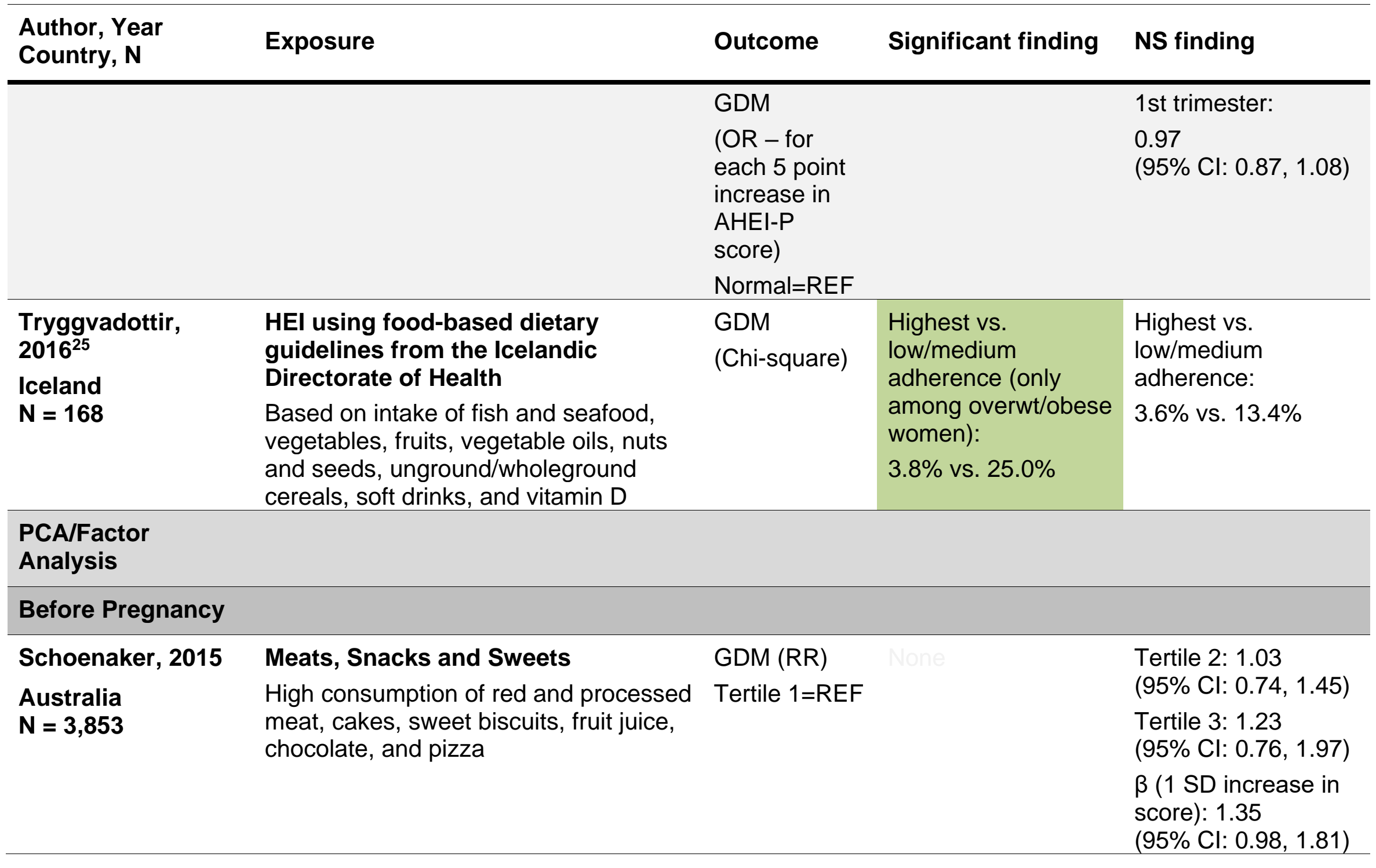

25 Tryggvadottir, 2016 is listed twice in the table: once under Index/Score and once under Principal Component Analysis / Factor Analysis 


\begin{tabular}{|c|c|c|c|c|}
\hline $\begin{array}{l}\text { Author, Year } \\
\text { Country, N }\end{array}$ & Exposure & Outcome & Significant finding & NS finding \\
\hline & $\begin{array}{l}\text { Mediterranean-Style } \\
\text { High factor loadings for vegetables, } \\
\text { legumes, nuts, tofu, rice, pasta, rye } \\
\text { bread, red wine, and fish }\end{array}$ & $\begin{array}{l}\text { GDM (RR) } \\
\text { Tertile 1=REF }\end{array}$ & $\begin{array}{l}\text { Tertile } 3: 0.56 \\
(95 \% \mathrm{Cl}: 0.41,0.77)\end{array}$ & $\begin{array}{l}\text { Tertile } 2: 0.97 \\
(95 \% \mathrm{Cl}: 0.75,1.28) \\
\beta(1 \mathrm{SD} \text { increase in } \\
\text { score }): 0.85 \\
(95 \% \mathrm{Cl}: 0.76,0.98)\end{array}$ \\
\hline & $\begin{array}{l}\text { Cooked Vegetables } \\
\text { High consumption of carrots, peas, } \\
\text { cooked potatoes, cauliflower, and } \\
\text { pumpkin }\end{array}$ & $\begin{array}{l}\text { GDM (RR) } \\
\text { Tertile 1=REF }\end{array}$ & & $\begin{array}{l}\text { Tertile } 2: 0.83 \\
(95 \% \mathrm{Cl}: 0.61,1.13) \\
\text { Tertile } 3: 1.04 \\
(95 \% \mathrm{Cl}: 0.77,1.38)\end{array}$ \\
\hline & $\begin{array}{l}\text { Western } \\
\text { High intake of red meat, processed } \\
\text { meat, refined grain products, sweets, } \\
\text { French fries and pizza }\end{array}$ & $\begin{array}{l}\text { GDM (RR) } \\
\text { Q1=REF }\end{array}$ & $\begin{array}{l}\text { Q5: } 1.63 \\
\text { (95\% Cl: } 1.20,2.21)\end{array}$ & $\begin{array}{l}\text { Q2: } 1.09 \\
\text { (95\% Cl: } 0.85,1.41) \\
\text { Q3: } 1.22 \\
(95 \% \mathrm{Cl}: 0.94,1.59) \\
\text { Q4: } 1.25 \\
(95 \% \mathrm{Cl}: 0.94,1.65)\end{array}$ \\
\hline
\end{tabular}




\begin{tabular}{|c|c|c|c|c|}
\hline $\begin{array}{l}\text { Author, Year } \\
\text { Country, N }\end{array}$ & Exposure & Outcome & Significant finding & NS finding \\
\hline \multirow{8}{*}{$\begin{array}{l}\text { He, } 2015 \\
\text { China } \\
N=3,063\end{array}$} & Vegetable & GDM (RR) & $\begin{array}{l}\text { Tertile 3: } 0.79 \\
(95 \% \mathrm{Cl}: 0.64,0.97)\end{array}$ & $\begin{array}{l}\text { Tertile } 2: 1.0 \\
(95 \% \mathrm{Cl}: 0.83,1.21)\end{array}$ \\
\hline & \multirow{3}{*}{$\begin{array}{l}\text { Protein-Rich } \\
\text { Freq intake of poultry, red meat, animal } \\
\text { organ meat, grains (mainly refined), } \\
\text { processed meat, fish, soups, leafy and } \\
\text { cruciferous vegetables, and eggs }\end{array}$} & \multirow{3}{*}{\multicolumn{2}{|c|}{$\begin{array}{l}\text { GDM }(R R) \\
\text { Tertile } 1=R E F\end{array}$}} & \multirow{3}{*}{$\begin{array}{l}\text { Tertile } 2: 0.99 \\
(95 \% \text { Cl: } 0.81,1.20) \\
\text { Tertile } 3: 0.95 \\
(95 \% \text { Cl: } 0.78,1.16)\end{array}$} \\
\hline & & & & \\
\hline & & & & \\
\hline & \multirow{2}{*}{$\begin{array}{l}\text { Prudent } \\
\text { Freq intake of dairy products, nuts, } \\
\text { eggs, fish, soups, fruits; infrequent } \\
\text { intake of processed meat, sugar- } \\
\text { sweetened beverages, and processed } \\
\text { vegetables }\end{array}$} & \multirow{2}{*}{\multicolumn{2}{|c|}{$\begin{array}{l}\text { GDM }(\mathrm{RR}) \\
\text { Tertile } 1=\mathrm{REF}\end{array}$}} & \multirow{2}{*}{$\begin{array}{l}\text { Tertile } 2: 0.96 \\
(95 \% \mathrm{Cl}: 0.78,1.17) \\
\text { Tertile } 3: 1.00 \\
(95 \% \mathrm{Cl}: 0.82,1.22)\end{array}$} \\
\hline & & & & \\
\hline & Sweet and Seafood & GDM (RR) & \multirow{2}{*}{$\begin{array}{l}\text { Tertile 3: } 1.23 \\
(95 \% \text { Cl: } 1.02,1.49)\end{array}$} & \multirow{2}{*}{$\begin{array}{l}\text { Tertile } 2: 0.84 \\
(95 \% \text { Cl: } 0.68,1.04)\end{array}$} \\
\hline & $\begin{array}{l}\text { Cantonese desserts, molluscs and } \\
\text { shellfish, and sugar-sweetened } \\
\text { beverages; low intakes of grains } \\
\text { (mainly refined) and leafy and } \\
\text { cruciferous vegetables }\end{array}$ & Tertile $1=$ REF & & \\
\hline
\end{tabular}




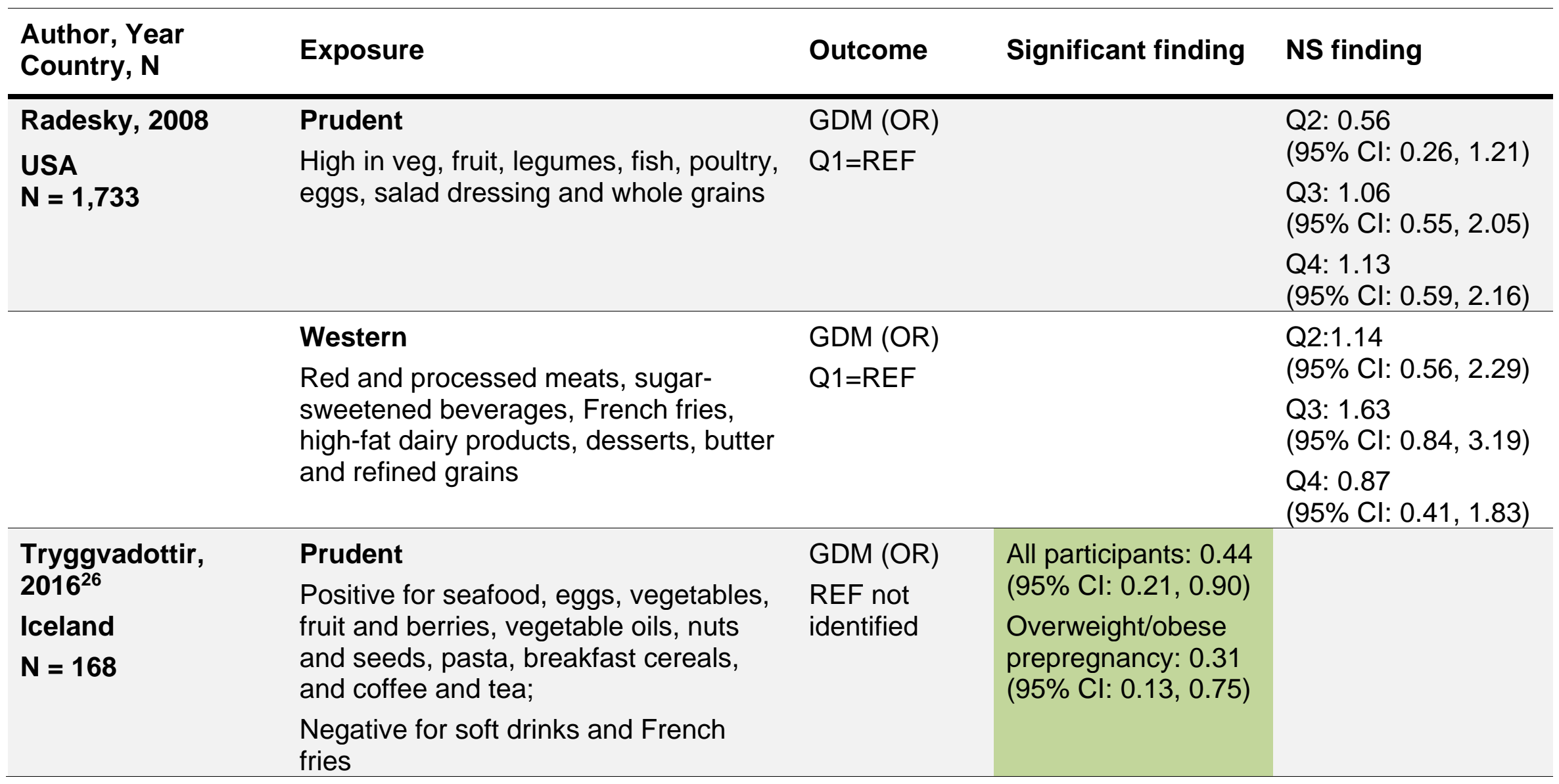

${ }^{26}$ Tryggvadottir, 2016 is listed twice in the table: once under Index/Score and once under Principal Component Analysis/Factor Analysis 


\begin{tabular}{|c|c|c|c|c|}
\hline $\begin{array}{l}\text { Author, Year } \\
\text { Country, N }\end{array}$ & Exposure & Outcome & Significant finding & NS finding \\
\hline & & $\begin{array}{l}\text { GDM } \\
\text { (Chi-square) }\end{array}$ & $\begin{array}{l}\text { Highest vs. } \\
\text { low/medium } \\
\text { adherence: } \\
1.8 \% \text { vs. } 14.3 \% \\
\text { Highest vs. } \\
\text { low/medium } \\
\text { adherence (only } \\
\text { overwt/obese } \\
\text { women): } \\
3.7 \% \text { vs. } 25.5 \%\end{array}$ & \\
\hline \multicolumn{5}{|l|}{ Other Method } \\
\hline \multicolumn{5}{|l|}{ Pregnancy } \\
\hline $\begin{array}{l}\text { Clapp, } 1998 \\
\text { USA } \\
N=12\end{array}$ & $\begin{array}{l}\text { Aboriginal Carbohydrate Diet } \\
\text { (Low-Gl) } \\
\text { Carbs from unprocessed whole grains, } \\
\text { fruits, beans, vegetables, and many } \\
\text { dairy products; includes most dense } \\
\text { whole grain and multigrain breads, bran } \\
\text { cereals, pastas, fresh fruits and } \\
\text { vegetables, yogurt, ice cream, and nuts }\end{array}$ & $\begin{array}{l}\text { Glycemic } \\
\text { response to } \\
\text { test meal } \\
\text { (mg/min) }\end{array}$ & & $\begin{array}{l}\text { Before pregnancy: } \\
10.8 \pm 5.4 \\
\text { vs } \\
\text { Late pregnancy: } \\
11.0 \pm 4.3\end{array}$ \\
\hline & $\begin{array}{l}\text { Cafeteria Carbohydrate Diet } \\
\text { (High-Gl) } \\
\text { Carbs from highly processed grains, } \\
\text { root vegetables, and simple sugars; } \\
\text { includes many highly refined breads, } \\
\text { potatoes, instant rice, most breakfast } \\
\text { cereals, desserts, and snack-type foods }\end{array}$ & $\begin{array}{l}\text { Glycemic } \\
\text { response to } \\
\text { test meal } \\
(\mathrm{mg} / \mathrm{min})\end{array}$ & $\begin{array}{l}\text { Before pregnancy: } \\
15.6 \pm 2.0 \\
\text { vs } \\
\text { Mid and late } \\
\text { pregnancy: } \\
27.9 \pm 4.1\end{array}$ & \\
\hline
\end{tabular}




\begin{tabular}{|c|c|c|c|c|}
\hline $\begin{array}{l}\text { Author, Year } \\
\text { Country, N }\end{array}$ & Exposure & Outcome & Significant finding & NS finding \\
\hline & \multirow[t]{6}{*}{ Aboriginal vs Cafeteria } & \multirow{6}{*}{$\begin{array}{l}\text { Average } \\
\text { serum insulin } \\
\text { response } \\
\text { over } 180 \text { min } \\
\text { to test meal } \\
(\mu \bigcup / m L / m i n)\end{array}$} & Before pregnancy: & \\
\hline & & & Early pregnancy: & \\
\hline & & & Mid pregnancy: & \\
\hline & & & $\begin{array}{l}\text { Aboriginal: } 23 \pm 5 \text { vs } \\
\text { Cafeteria: } 39 \pm 6\end{array}$ & \\
\hline & & & Late pregnancy: & \\
\hline & & & $\begin{array}{l}\text { Aboriginal: } 31 \pm 5 \text { vs } \\
\text { Cafeteria: } 54 \pm 8\end{array}$ & \\
\hline
\end{tabular}




\section{Assessment of the body of evidence}

This body of evidence was deemed to be limited in strength. The individual grading elements are discussed below.

\section{Internal validity (determined with NEL Bias Assessment Tool):}

- The data were primarily observational in nature, making it difficult to determine causal effect of the dietary patterns.

- The use of self-reported exposure and outcome measurement introduces potential measurement error and limits the validity of these data.

- FFQ were the primary measurement tool, and both the self-reported data obtained with this tool and the variability between studies in the type of $F F Q$ used (i.e., number of items assessed, whether or not it is validated in a pregnant sample) affected the validity of these data.

- Key confounders such as parity, educational attainment, smoking status, race/ethnicity, maternal age, family poverty income ratio, pre-pregnancy BMI, mean total energy intake, family history of diabetes, history of GDM, diagnosis of pre-diabetes and PCOS were not consistently controlled across studies.

- Multiple studies did not report data on key confounding factors at baseline and did not control for potential differences across dietary groups in the analyses.

\section{Adequacy:}

- The number of articles in this body of evidence is modest. This evidence base includes 11 studies corresponding to 1 RCT and 6 unique cohorts. Notably, data from NHS II are represented in 3 studies $(8,10,11)$, and data from ALSWH $(6,7)$ and Project Viva $(4,5)$ are represented in 2 studies each.

- The sample sizes varied considerably, ranging from 12 to 15,254 subjects in the NHS II, and most of the studies, except Karamanos et al., did not report power calculations. This limits the ability to draw meaningful conclusions.

- Sub-groups such as women of lower SES and different race/ethnicities were not adequately represented in this evidence base.

- The small number of unique research groups included limits the adequacy of this evidence base to answer the research question.

\section{Consistency:}

- There was moderate consistency across the body of evidence suggesting that adherence to "healthier" dietary patterns (defined different ways) is likely to be associated with better outcomes (gestational diabetes, glucose intolerance, insulin resistance, blood glucose levels, HOMA-IR).

- Specifically, a dietary pattern characterized by vegetables, fruits, whole grains, nuts, legumes and fish and lower in red and processed meat was associated with better glucose tolerance and reduced risk of GDM.

- When stratified by the time period of dietary pattern assessment, greater adherence to a dietary pattern 2-10 years before pregnancy showed a consistent inverse association with GDM risk $(6-8,10,11)$. In addition to the consistency within the body of evidence, findings from this review are in agreement with similar research conducted in the non-pregnant populations. For example, many studies have found that adherence to a healthy dietary 
pattern is associated with a lower plasma insulin, HOMA-IR 272829.

- Although studies in this review measured diet during a wider time window of 2 to 10 years prior to pregnancy, other longitudinal studies have reported that maternal diet remains relatively stable from pre-pregnancy through pregnancy, thus supporting the validity of these findings 303132 .

- There were mixed findings when looking at studies that assessed diet during pregnancy: three studies showed an inverse association $(2,3,9)$, one study showed an inverse association only with blood glucose but not with GDM (5), one study showed an effect on blood glucose and insulin response but did not study GDM (1), and one other study showed no association (4).

\section{Impact:}

- Almost all the studies in the body of evidence directly examined the relationship between different dietary patterns or different levels of adherence to a dietary pattern and GDM. However, only two cohorts $(3,9)$ and one pilot trial $(1)$ were designed specifically to assess this relationship.

- Modest risk reduction was observed across studies that found a significant relationship between adhering to a "healthy" dietary pattern and risk of GDM.

- Highest adherence to a "healthy" dietary pattern before and during pregnancy was associated with a GDM risk reduction of $24 \%$ to $56 \%$. Categorized another way, lower adherence to a "healthy" dietary pattern was associated with $35 \%$ to $37 \%$ increased GDM risk.

- Greater adherence to an "unhealthy" dietary pattern was associated with an increase in risk of $23 \%$ to $63 \%$.

\section{Generalizability:}

- Only half of the studies $(n=6)$ in this body of evidence were conducted in the U.S. Even the studies conducted in the U.S. had limited generalizability because the subjects predominantly represented Caucasian women with access to health care.

- Minority and lower SES populations were underrepresented in these data. It is unknown if the findings would be applicable in more diverse samples before or during pregnancy.

\section{Other limitations/considerations:}

The studies included in this review have a number of other limitations that make interpretation of results challenging:

\footnotetext{
${ }^{27}$ Wang, Z., Adair, L. S., Cai, J., Gordon-Larsen, P., Siega-Riz, A. M., Zhang, B., \& Popkin, B. M. (2017). Diet Quality Is Linked to Insulin Resistance among Adults in China. J Nutr. doi:10.3945/jn.117.256180

${ }^{28}$ Schulze, M. B., Hoffmann, K., Manson, J. E., Willett, W. C., Meigs, J. B., Weikert, C., . . Hu, F. B. (2005). Dietary pattern, inflammation, and incidence of type 2 diabetes in women. Am J Clin Nutr, 82(3), 675-684; quiz 714-675.

${ }_{29}$ U.S. Department of Health and Human Services and U.S. Department of Agriculture (2015). 2015 - 2020 Dietary Guidelines for Americans. 8th Edition. Retrieved from https://health.gov/dietaryguidelines/2015/guidelines/.

30 Crozier, S. R., Robinson, S. M., Godfrey, K. M., Cooper, C., \& Inskip, H. M. (2009). Women's dietary patterns change little from before to during pregnancy. J Nutr, 139(10), 1956-1963. doi:10.3945/jn.109.109579

31 Olson, C. M. (2005). Tracking of food choices across the transition to motherhood. J Nutr Educ Behav, 37(3), 129-136.

${ }^{32}$ Cuco, G., Fernandez-Ballart, J., Sala, J., Viladrich, C., Iranzo, R., Vila, J., \& Arija, V. (2006). Dietary patterns and associated lifestyles in preconception, pregnancy and postpartum. Eur J Clin Nutr, 60(3), 364-371.

doi:10.1038/sj.ejcn.1602324
} 
- A wide range of methods (sometimes using the same nomenclature) was used to define and assess dietary patterns, which made it difficult to compare and contrast results across studies. Journal editors and peer-reviewers may be less willing to publish studies that replicate others' findings, which could have resulted in an evidence base with a wide array of dietary patterns. It is important for the editors and peer-reviewers to understand the need for publishing studies that replicate dietary patterns, in addition to publishing studies that assess unique dietary patterns ${ }^{33}$.

- There was heterogeneity in terms of when dietary data were assessed. Half of the studies measured diet before pregnancy (2-10 years prior to pregnancy) while the rest assessed diet during pregnancy (with a recall period ranging from 4 days to few months).

- In some studies, the time point of exposure assessment was very close to when the outcome was assessed, even though these studies clarified temporality (2, 9).

- Interaction with BMI was not assessed. Only some studies stratified results by BMI.

- Different criteria (e.g. ADA, IADPSG) were used across studies to define GDM.

\section{Research recommendations}

To assess the relationship between dietary patterns before and during pregnancy and risk of GDM more adequately, additional research is needed that should:

- Include diverse populations from the U.S. and elsewhere with varying racial/ethnic and socioeconomic backgrounds.

- Foster collaborative efforts across different regions and populations so that dietary patterns can be more consistently scored, compared and reproduced across studies.

- Develop and validate novel epidemiological tools that can accurately capture the complexity of dietary habits.

- Promote harmonization of research methods across various cohorts and randomized trials, similar to the National Cancer Institute's Dietary Patterns Methods Project ${ }^{34}$.

- Adjust for key confounding factors in observational studies, including parity, educational attainment, smoking status, race/ethnicity, maternal age, family poverty income ratio, pre-pregnancy BMI, mean total energy intake, family history of diabetes, previous diagnosis of pre-diabetes and PCOS.

- Improve comparability across studies by increasing the uniformity of 1) timing of dietary assessment, and 2) outcome measured (avoiding self-report).

- Include well-designed and sufficiently powered RCTs.

- Include and assess effect measure modification by pre-pregnancy BMI and fetal sex.

\footnotetext{
33 U.S. Department of Health and Human Services and U.S. Department of Agriculture. (2015). 2015-2020 Dietary Guidelines for Americans. 8th Edition. Retrieved from https://health.gov/dietaryguidelines/2015/guidelines/.

${ }^{34}$ Liese, A. D., Krebs-Smith, S. M., Subar, A. F., George, S. M., Harmon, B. E., Neuhouser, M. L., . . Reedy, J.

(2015). The Dietary Patterns Methods Project: synthesis of findings across cohorts and relevance to dietary guidance. J Nutr, 145(3), 393-402. doi:10.3945/jn.114.205336
} 


\section{Included articles}

1. Clapp JF, 3rd. Effect of dietary carbohydrate on the glucose and insulin response to mixed caloric intake and exercise in both nonpregnant and pregnant women. Diabetes Care 1998;21 Suppl 2:B107-12.

2. He JR, Yuan MY, Chen NN, Lu JH, Hu CY, Mai WB, Zhang RF, Pan YH, Qiu L, Wu YF, et al. Maternal dietary patterns and gestational diabetes mellitus: a large prospective cohort study in China. Br J Nutr 2015;113(8):1292-300.

3. Karamanos B, Thanopoulou A, Anastasiou E, Assaad-Khalil S, Albache N, Bachaoui M, Slama CB, El Ghomari H, Jotic A, Lalic N, et al. Relation of the Mediterranean diet with the incidence of gestational diabetes. Eur J Clin Nutr 2014;68(1):8-13.

4. Radesky JS, Oken E, Rifas-Shiman SL, Kleinman KP, Rich-Edwards JW, Gillman MW. Diet during early pregnancy and development of gestational diabetes.

Paediatr Perinat Epidemiol 2008;22(1):47-59.

5. Rifas-Shiman SL, Rich-Edwards JW, Kleinman KP, Oken E, Gillman MW. Dietary quality during pregnancy varies by maternal characteristics in Project Viva: a US cohort. J Am Diet Assoc 2009;109(6):1004-11.

6. Schoenaker DA, Soedamah-Muthu SS, Callaway LK, Mishra GD. Pre-pregnancy dietary patterns and risk of gestational diabetes mellitus: results from an Australian population-based prospective cohort study. Diabetologia 2015;58(12):2726-35.

7. Schoenaker DA, Soedamah-Muthu SS, Mishra GD. Quantifying the mediating effect of body mass index on the relation between a Mediterranean diet and development of maternal pregnancy complications: the Australian Longitudinal Study on Women's Health. Am J Clin Nutr 2016;104(3):638-45.

8. Tobias DK, Zhang C, Chavarro J, Bowers K, Rich-Edwards J, Rosner B, Mozaffarian D, Hu FB. Prepregnancy adherence to dietary patterns and lower risk of gestational diabetes mellitus. Am J Clin Nutr 2012;96(2):289-95.

9. Tryggvadottir EA, Medek H, Birgisdottir BE, Geirsson RT, Gunnarsdottir I. Association between healthy maternal dietary pattern and risk for gestational diabetes mellitus. Eur J Clin Nutr 2016;70(2):237-42.

10. Zhang C, Schulze MB, Solomon CG, Hu FB. A prospective study of dietary patterns, meat intake and the risk of gestational diabetes mellitus. Diabetologia 2006;49(11):2604-13.

11. Zhang C, Tobias DK, Chavarro JE, Bao W, Wang D, Ley SH, Hu FB. Adherence to healthy lifestyle and risk of gestational diabetes mellitus: prospective cohort study. BMJ 2014;349:g5450. 


\section{ANALYTIC FRAMEWORK}

The analytic framework (Figure 1) illustrates the overall scope of the systematic review, including the population, the interventions and/or exposures, comparators, and outcomes of interest. It also includes definitions of key terms and identifies key confounders considered in the systematic review. This is the analytic framework for the systematic review conducted to examine the relationship between dietary patterns before and during pregnancy and risk of gestational diabetes mellitus.

\section{Figure 1: Analytic framework}

Analytic Framework: What is the relationship between dietary patterns before and during pregnancy and risk of gestational diabetes?

\section{Target Population}

Women preconception and during pregnancy (1st, 2nd and 3rd trimesters)

\section{Intervention/Exposure \\ Dietary pattern assessed using \\ - Indices \& scores \\ - Factor \& cluster analysis \\ - Reduced rank regression \\ - Other methods \\ Comparator}

Different levels of adherence to a dietary pattern; Adherence to a different dietary pattern

\begin{tabular}{l} 
Intermediate Outcomes \\
- Glucose intolerance \\
- Insulin resistance \\
- HbA1C \\
- HOMA-IR \\
- Orasting blood glucose \\
\hline
\end{tabular}

Endpoint Health Outcomes

\section{Key Definitions}

- Dietary patterns: the quantities, proportions, variety or combinations of different foods and beverages in diets, and the frequency with which they are habitually consumed - Gestational diabetes: diabetes occurring during pregnancy in women not previously diagnosed with diabetes

\section{Key Confounders}

- Parity

- Educational attainment

- Smoking status

- Race/ethnicity

- Maternal age

- Family poverty income ratio

- Pre-pregnancy BMI

- Mean total energy intake

- Family history of diabetes

- Previous diagnosis of pre-diabetes

- Polycystic ovarian syndrome 


\section{SEARCH PLAN AND RESULTS}

\section{Inclusion and exclusion criteria}

The inclusion and exclusion criteria are a set of characteristics to determine which studies will be included or excluded in the systematic review. Table 6 provides the inclusion and exclusion criteria for the systematic review question(s): What is the relationship between dietary patterns before and during pregnancy and risk of gestational diabetes mellitus?

\section{Table 6. Inclusion and exclusion criteria}

\begin{tabular}{|c|c|c|}
\hline Category & Inclusion Criteria & Exclusion Criteria \\
\hline $\begin{array}{l}\text { Study } \\
\text { Design }\end{array}$ & $\begin{array}{l}\text { - Randomized controlled trials } \\
\text { - Prospective cohort studies } \\
\text { - Retrospective cohort studies } \\
\text { - }\end{array}$ & $\begin{array}{l}\text { - Non-randomized controlled } \\
\text { trials } \\
\text { - Cross-sectional studies } \\
\text { - Case-control studies } \\
\text { - Uncontrolled studies } \\
\text { - Pre/post studies with a control } \\
\text { - Pre/post studies without a } \\
\text { - } \text { Control } \\
\text { - Sarrative reviews } \\
\text { - Meta-analyses }\end{array}$ \\
\hline $\begin{array}{l}\text { Exposure/ } \\
\text { Intervention }\end{array}$ & $\begin{array}{l}\text { - Studies that provide a description } \\
\text { of the dietary pattern(s) (i.e., foods } \\
\text { and beverages) consumed by } \\
\text { subjects and that methodologically } \\
\text { use: } \\
\circ \text { Indices \& scores } \\
\circ \text { Cluster or factor analysis } \\
\circ \text { Reduced rank regression } \\
\circ \text { Other methods }\end{array}$ & $\begin{array}{l}\text { - Studies that do not provide a } \\
\text { description of the dietary } \\
\text { pattern(s) (i.e., foods and } \\
\text { beverages) consumed by }^{\text {subjects }^{35}}\end{array}$ \\
\hline Comparator & $\begin{array}{l}\text { - Different levels of adherence to a } \\
\text { dietary pattern } \\
\text { - Adherence to a different dietary } \\
\text { pattern }\end{array}$ & \\
\hline
\end{tabular}

35 For example, a study would be excluded from the systematic review if the dietary pattern were labeled "vegetarian" but lacked a description of what foods/beverages were consumed as part of that dietary pattern. 


\begin{tabular}{|c|c|c|}
\hline Category & Inclusion Criteria & Exclusion Criteria \\
\hline Date Range & $\begin{array}{l}\text { - Studies published in the following } \\
\text { date range: 1980-present (search } \\
\text { date) }\end{array}$ & \\
\hline Language & - Studies published in English & $\begin{array}{l}\text { - Studies published in } \\
\text { languages other than English }\end{array}$ \\
\hline \multirow[t]{2}{*}{$\begin{array}{l}\text { Study } \\
\text { Setting }\end{array}$} & $\begin{array}{l}\text { - Studies conducted in Very High } \\
\text { and High Human Development } \\
\text { Countries* }^{*}\end{array}$ & $\begin{array}{l}\text { - Studies conducted in Medium } \\
\text { and Low Human } \\
\text { Development Countries* }\end{array}$ \\
\hline & $\begin{array}{l}\text { *Determined using the most recent } \\
\text { Human Development Index }\end{array}$ & $\begin{array}{l}\text { *Determined using the most } \\
\text { recent Human Development } \\
\text { Index }\end{array}$ \\
\hline $\begin{array}{l}\text { Study } \\
\text { Duration }\end{array}$ & - Studies regardless of length & \\
\hline Temporality & $\begin{array}{l}\text { Studies when the exposure was } \\
\text { assessed prior to the outcome } \\
\text { Studies when exposure and } \\
\text { outcome assessment occurred } \\
\text { during overlapping time periods, } \\
\text { as long as the study explicitly } \\
\text { states that the exposure was } \\
\text { assessed prior to the outcome }\end{array}$ & $\begin{array}{l}\text { - Studies when the outcome } \\
\text { was assessed prior to the } \\
\text { exposure } \\
\text { - Studies when exposure and } \\
\text { outcome assessment } \\
\text { occurred during overlapping } \\
\text { time periods and the study } \\
\text { does not explicitly state that } \\
\text { the exposure was assessed } \\
\text { prior to the outcome }\end{array}$ \\
\hline $\begin{array}{l}\text { Publication } \\
\text { Status }\end{array}$ & $\begin{array}{l}\text { - Studies published in peer- } \\
\text { reviewed journals }\end{array}$ & $\begin{array}{l}\text { Grey literature, } \\
\text { including unpublished } \\
\text { data, manuscripts, } \\
\text { reports, abstracts, } \\
\text { conference } \\
\text { proceedings }\end{array}$ \\
\hline $\begin{array}{l}\text { Study } \\
\text { Subjects }\end{array}$ & $\begin{array}{l}\text { - Human subjects } \\
\text { - Adolescent girls and women } \\
\text { capable of becoming pregnant } \\
\text { (15-44 years) } \\
\text { - Pregnant girls and women (15-44 } \\
\text { years) - single and multiple } \\
\text { pregnancies } \\
\text { - Neonates }\end{array}$ & $\begin{array}{l}\text { - Animal and in vitro models } \\
\text { - Hospitalized patients, when } \\
\text { hospitalization is not related } \\
\text { to pregnancy, birth and } \\
\text { immediate postpartum } \\
\text { - Pregnancies } \\
\text { conceived ONLY } \\
\text { using Assisted } \\
\text { Reproductive } \\
\text { Technologies }\end{array}$ \\
\hline
\end{tabular}




\begin{tabular}{|c|c|c|}
\hline Category & Inclusion Criteria & Exclusion Criteria \\
\hline $\begin{array}{l}\text { Size of } \\
\text { Study } \\
\text { Groups }\end{array}$ & $\begin{array}{l}\text { - Studies regardless of } \\
\text { group size }\end{array}$ & \\
\hline $\begin{array}{l}\text { Health } \\
\text { Status of } \\
\text { Study } \\
\text { Subjects }\end{array}$ & $\begin{array}{l}\text { - Studies conducted in generally } \\
\text { healthy women of reproductive } \\
\text { age, including women in pre/peri- } \\
\text { conception and pregnancy } \\
\text { - Studies conducted in samples with } \\
\text { elevated chronic disease risk or } \\
\text { pregnancy related conditions, or } \\
\text { that enroll some subjects with a } \\
\text { disease or with health outcome of } \\
\text { interest such as } \\
\text { O Anemia } \\
\circ \text { Gestational diabetes } \\
\circ \text { Hypertension } \\
\circ \text { Preeclampsia } \\
\circ \text { Hyperemesis Gravidarum } \\
\text { O Previous adverse outcome } \\
\text { (e.g., preterm) } \\
\text { Obesity }\end{array}$ & $\begin{array}{l}\text { - Studies that exclusively enroll } \\
\text { subjects with chronic } \\
\text { conditions (e.g. hypertension, } \\
\text { diabetes) that are not related } \\
\text { to the index pregnancy } \\
\text { - Studies that exclusively enroll } \\
\text { subjects with a disease or } \\
\text { with the health outcome of } \\
\text { interest (intermediate or } \\
\text { endpoint health outcomes) } \\
\text { - Studies done in } \\
\text { hospitalized or } \\
\text { malnourished } \\
\text { subjects, if } \\
\text { hospitalization is not } \\
\text { related to index } \\
\text { pregnancy }\end{array}$ \\
\hline Outcomes & $\begin{array}{l}\text { - Gestational diabetes mellitus } \\
\text { - Intermediate Outcomes: } \\
\circ \text { Glucose intolerance } \\
\circ \text { Insulin resistance } \\
\circ \text { HbA1C } \\
\circ \text { HOMA-IR } \\
\circ \text { Fasting blood glucose } \\
\text { - Oral glucose tolerance test }\end{array}$ & \\
\hline
\end{tabular}




\title{
Search terms and electronic databases used
}

\author{
PubMed, US National Library of Medicine
}

- Date(s) searched: January 1980 to January 2017

- Search Terms:

pregnancy[mh] OR "Prenatal Exposure Delayed Effects"[mesh] OR "Maternal Exposure"[mesh] OR "pregnant women"[mh] OR pregnan*[tiab] OR prenatal[tiab] OR maternal OR mother* OR postpartum OR newborn*[tiab] OR perinatal OR peri-natal OR pre-conception OR preconception OR periconception OR periconception OR "Infant, Newborn"[Mesh] OR neonat*[tiab] OR newly born* OR "Peripartum Period"[Mesh] OR peripartum[tiab] OR peripartum[tiab] OR gestation* OR natal OR puerperium[tiab] OR "Maternal Nutritional Physiological Phenomena"[Mesh]

\section{AND}

hypertensi*[tiab] OR "Hypertension"[Mesh:NoExp] OR vomit* OR diabetes*[tiab] OR diabetic*[tiab] OR "Birth Weight"[Mesh] OR "Birth Weight"[tiab] OR "Glucose Intolerance"[Mesh] OR Glucose Intoleran*[tiab] OR glucose toleran* OR "Insulin Resistance"[Mesh] OR Insulin Resistan*[tiab] OR Dysglycemia[tiab] OR fasting blood glucose* OR "Hemoglobin A, Glycosylated"[Mesh] OR "Proteinuria"[Mesh:noexp] OR Albuminuria OR "Blood Pressure"[mh] OR "blood pressure"[tiab]

OR

"Diabetes, Gestational"[Mesh] OR (gestation*[tiab] AND (diabetes*[tiab] OR diabetic*[tiab])) OR "Pre-Eclampsia"[Mesh] OR "Pre-Eclampsia"[tiab] OR preeclampsia[tiab] OR "Hypertension, Pregnancy-Induced"[Mesh] OR Eclampsia OR "Gestational Age"[Mesh] OR "Morning Sickness"[Mesh] OR (Hyperemesis Gravidarum) OR "Gestational Age"[tiab] OR "Obstetric Labor, Premature"[Mesh] OR ((prematur*[tiab] OR preterm [tiab]) AND (baby[tiab] OR infant*[tiab] OR birth OR labor OR membrane* OR babies)) OR "Fetal Growth Retardation"[Mesh] OR IUGR[tiab] OR "Intrauterine growth restriction" OR "Fetal Development"[Mesh:noexp] OR "Fetal Weight"[Mesh] OR "Umbilical Arteries"[Mesh] OR "Uterine Artery"[Mesh]

AND

("diet quality" OR dietary pattern* OR diet pattern* OR eating pattern* OR food pattern* OR eating habit* OR dietary habit* OR food habit* OR dietary profile* OR food profile* OR diet profile* OR eating profile* OR dietary guideline* OR dietary recommendation* OR eating style* OR

(DASH[ti] OR DASH[tw] OR ("dietary approaches"[ti] AND hypertension[ti]) OR "Diet, Mediterranean"[Mesh] OR Mediterranean[ti] OR vegan* OR vegetarian* OR "Diet, Vegetarian"[Mesh] OR "prudent diet" OR "western diet" OR nordiet OR omni[ti] OR omniheart[tiab] OR (Optimal Macronutrient Intake Trial to Prevent Heart Disease) OR adventist* OR ((Okinawa* OR "Ethnic Groups"[Mesh] OR "plant based" OR Mediterranean[tiab] OR Nordic[tiab] OR "heart healthy"[tiab] OR indo-mediterranean) AND (diet[mh] OR diet[tiab] OR diets[tiab] OR 
food[mh]))) OR

("Guideline Adherence"[Mesh] AND (diet OR food OR eating OR eat OR dietary OR feeding OR nutrition OR nutrient $\left.{ }^{\star}\right)$ ) OR (adherence AND (nutrient* OR nutrition OR diet OR dietary OR food OR eat OR eating) AND (guideline* OR guidance OR recommendation $\left.{ }^{*}\right)$ ) OR

(dietary score* OR adequacy index* OR kidmed OR Diet Quality Index* OR Food Score* OR Diet Score* OR MedDietScore OR Dietary Pattern Score* OR "healthy eating index") OR

((index*[ti] OR score*[ti] OR indexes OR scoring[ti] OR indices[ti]) AND (dietary[ti] OR nutrient*[ti] OR eating[tiab] OR food[ti] OR food[mh] OR diet[ti] OR $\operatorname{diet}[\mathrm{mh}])$ AND (pattern* OR habit* OR profile*)) OR meals[mh] OR meals[tiab] OR meal[tiab] OR mealtime* ${ }^{*}$ tiab]

OR

$\operatorname{diet}[\mathrm{mh}: n o e x p]$ OR $\operatorname{diet}[\mathrm{ti}]$ OR $\operatorname{diets[ti]}$ OR food"[tiab] OR "Food"[mh:noexp] OR "Eating"[mh] OR dietary intake*[tiab] OR food intake*[tiab] OR food habits[mh] OR diet habit* ${ }^{\star}\left[\right.$ tiab] OR eating habit* ${ }^{*}$ tiab] OR food choice*[tiab] OR dietary choice*[tiab] OR dietary change*[tiab] NOT (editorial[ptyp] OR comment[ptyp] OR news[ptyp] OR letter[ptyp] OR review[ptyp] OR systematic[sb])

\section{Embase, Elsevier:}

- Date(s) searched: January 1980 to January 2017

- Search Terms:

'pregnancy'/exp OR 'pregnant woman'/exp OR 'prenatal period'/exp OR 'mother'/exp OR 'prenatal exposure'/exp OR 'prenatal growth'/exp OR 'puerperium'/exp OR 'newborn'/exp OR prematurity/exp OR pregnan*:ti,ab OR maternal:ti,ab OR mother*:ti,ab OR prenatal:ti,ab OR pre-natal:ti,ab OR 'puerperium':ti,ab OR postpartum:ti,ab OR newborn:ti,ab OR neonat*:ti,ab OR "newly born":ti,ab OR periconception:ti,ab OR peri-conception:ti,ab OR preconception:ti,ab OR preconception;ti,ab OR gestation* OR peripartum:ti,ab OR peri-partum:ti,ab OR natal:ti,ab OR gestation* OR 'perinatal development'/exp OR 'perinatal care'/de OR perinatal:ti,ab OR peri-natal:ti,ab OR 'puerperium'/de OR 'puerperium':ti,ab OR 'maternal nutrition'/exp

AND

hypertensi* OR hyperemesis:ti,ab OR vomit*:ti,ab OR diabet* OR 'birth weight'/exp OR birthweight:ti,ab OR ((neonatal OR newborn) NEAR/3 weight)

OR

'glucose intolerance'/exp OR (Glucose NEAR/2 Intoleran*) OR (glucose NEAR/2 toleran*) OR 'insulin resistance'/exp OR (Insulin NEAR/1 Resistan*):ti,ab OR Dysglycemia OR "fasting blood glucose" OR 'hemoglobin A1c'/exp OR 'hemoglobin A1c' OR 'proteinuria'/exp OR albuminuria OR "Blood Pressure"/de OR

'pregnancy diabetes mellitus'/exp OR “diabetes mellitus gravidarum":ti,ab OR 
'eclampsia OR preeclampsia'/exp OR eclampsia:ti,ab OR preeclampsia:ti,ab OR pre-eclampsia:ti,ab OR 'maternal hypertension'/exp OR 'gestational age'/exp OR 'small for date infant'/exp OR 'gestational age' OR 'hyperemesis gravidarum'/exp OR 'morning sickness'/exp OR (gestation* NEAR/2 diabet $^{\star}$ ):ti,ab OR (Obstetric NEAR/3 (Labor OR labour)) OR (labor/exp AND

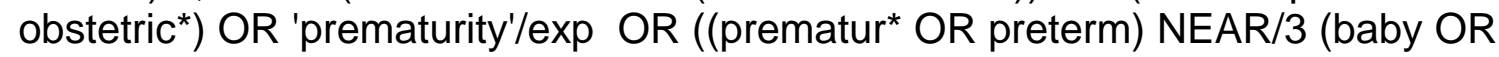
infant* OR babies OR birth OR childbirth OR labor OR membrane*)) OR 'intrauterine growth retardation'/de OR IUGR:ti,ab OR "Intrauterine growth restriction" OR 'fetus growth'/exp OR 'fetus development'/exp OR 'fetus weight'/exp OR 'umbilical artery'/exp OR 'uterine artery'/exp OR ((fetal OR fetus OR foetal OR foetus OR embryo*) NEAR/3 (weight OR develop* OR growth)):ti,ab

\section{AND}

'eating habit'/exp OR 'Mediterranean diet'/exp OR nordiet:ti,ab OR 'nordic diet':ti,ab OR DASH:ti,ab OR 'dietary approaches to stop hypertension':ti,ab OR vegan*:ab,ti OR vegetarian*:ab,ti OR 'vegetarian diet'/exp OR 'vegetarian'/exp OR 'prudent diet':ti,ab OR 'western diet':ti,ab OR 'Western diet'/exp OR meal/de OR omniheart:ti, ab OR omni:ti OR 'plant based diet' OR ((eating OR food OR diet $^{\star}$ OR calori ${ }^{\star}$ ) NEAR/3 (pattern? OR habit? OR profile? OR recommendation? OR guideline? OR style* OR choice* OR intake OR quality)) OR (('ethnic, racial and religious groups'/exp OR Okinawa* OR adventist* OR 'mediterranean') AND (diet/exp OR eating/exp OR 'food intake'/de OR calori* OR diet* OR food OR eating))

\section{OR}

Diet/de OR 'dietary intake'/de OR 'food preference'/de OR 'food intake'/de OR 'diet restriction'/exp OR 'eating habit'/exp OR diet*:ti OR kidmed:ab,ti OR 'meddietscore':ab,ti OR 'healthy eating index':ab,ti OR ((index OR score OR scoring OR indices) NEAR/3 (diet* OR eating OR food)) OR "food consumption"

OR

food $^{*}:$ ti,ab OR "Food"/de OR Eating:ti,ab OR (dietary NEAR/1 change*):ti,ab OR Meal ${ }^{*}: \mathrm{ti}, \mathrm{ab}$

\section{Cochrane, Central Register of Controlled Trials, John Wiley \& Sons:}

- Date(s) searched: January 1980 to January 2017

- Search Terms:

[mh pregnancy] OR [mh "Maternal Exposure"] OR [mh "Prenatal Exposure Delayed Effects"] OR [mh "pregnant women"] OR pregnan*:ti,ab OR prenatal OR maternal OR mother* OR postpartum OR newborn*:ti,ab OR perinatal OR perinatal OR pre-conception OR preconception OR peri-conception OR periconception OR [mh "Infant, Newborn"] OR neonat*:ti,ab OR (newly NEAR/1 born*) OR gestation* OR peripartum OR peri-partum OR natal:ti,ab OR puerperium OR gravidarum OR [mh "Peripartum Period"] OR peripartum:ti,ab 
OR peri-partum:ti,ab OR natal OR puerperium:ti,ab OR [mh "Maternal Nutritional Physiological Phenomena"]

AND

(hypertensi":ti,ab OR [mh ^Hypertension] OR vomit* $:$ ti,ab OR diabet*:ti,ab OR [mh "Birth Weight"] OR "Birth Weight":ti, ab OR [mh "Glucose Intolerance"] OR (Glucose NEAR/1 Intoleran*) OR (glucose NEAR/1 toleran*) OR [mh "Insulin Resistance"] OR (Insulin NEAR/1 Resistan*:ti,ab) OR Dysglycemia:ti,ab OR "fasting blood glucose" OR [mh "Hemoglobin A, Glycosylated"] OR [mh ^"Proteinuria"] OR Albuminuria OR [mh "Blood Pressure"] OR "blood pressure":ti,ab)

OR

[mh "Diabetes, Gestational"] OR (gestation* NEAR/1 diabet*) OR [mh "PreEclampsia"] OR "Pre-Eclampsia":ti,ab OR preeclampsia:ti,ab OR [mh "Hypertension, Pregnancy-Induced"] OR Eclampsia OR [mh "Gestational Age"] OR [mh "Morning Sickness"] OR (Hyperemesis NEAR/3 Gravidarum) OR "Gestational Age":ti,ab OR [mh "Birth Weight"] OR "Birth Weight":ti,ab OR ((neonatal OR newborn) NEAR/3 weight) OR [mh "Obstetric Labor, Premature"] OR ((prematur*:ti,ab OR preterm:ti,ab) AND (baby:ti,ab OR infant*:ti,ab OR birth OR labor OR membrane* OR babies)) OR [mh "Fetal Growth Retardation"] OR IUGR:ti,ab OR "Intrauterine growth restriction" OR [mh ^"Fetal Development"] OR [mh "Fetal Weight"] OR [mh "Umbilical Arteries"] OR [mh "Uterine Artery"]

AND (diet:ti OR diets:ti OR dietary:ti OR meal*:ti,ab OR "prudent diet" OR nordiet:ti,ab OR omniheart OR "Optimal Macronutrient Intake Trial to Prevent Heart Disease" OR ((Index OR score OR indices OR scoring) NEAR/3 (dietary OR diet OR food OR eating)) OR "adequacy index" OR kidmed OR MedDietScore)

OR 'dietary approaches to stop hypertension':ti,ab OR omniheart:ti,ab OR omni:ti OR 'plant based diet' OR ((eating OR food OR diet* OR calori*) NEAR/3 (pattern? OR habit? OR profile? OR recommendation? OR guideline? OR style* OR choice* OR intake OR quality))

OR

food $^{*}:$ ti,ab OR Eating:ti,ab OR (dietary NEAR/1 change*):ti,ab OR DASH:ti,ab OR vegan*:ab,ti OR vegetarian*:ab,ti OR omni:ti OR ((ethni* OR racial OR religio* OR asia* OR western OR Okinawa* OR adventist* OR 'mediterranean' OR Nordic* OR indo-mediterranean) NEAR/3 (calori* OR diet $^{\star}$ OR food OR eating))

OR [mh "Diet, Mediterranean"] OR [mh "Diet, Vegetarian"] OR ([mh "Ethnic Groups"] AND ([mh diet] OR diet*:ti,ab OR [mh ^food] OR eat:ti,ab OR eating:ti,ab OR [mh "Eating"] OR [mh "food habits"])) OR

([mh "Guideline Adherence"] AND (diet OR food OR eating OR eat OR dietary)) OR ((adhere* OR adhering) AND (diet OR dietary OR food OR eat OR eating) AND (guideline* OR guidance OR recommendation $\left.{ }^{\star}\right)$ ) OR 
[mh meals] OR [mh ^diet] OR diet*:ti,ab OR [mh ^"Food"] OR [mh "Eating"] OR [mh "food habits"]

\section{CINAHL (Plus) with Full Text, EBSCO (Cumulative Index to Nursing and Allied Health Literature):}

- Date(s) searched: January 1980 to January 2017

- Search Terms:

(MH "Food and Beverages") OR (MH "Food") OR (MH "Diet") OR (MH "Eating") OR (MH "Eating Behavior") OR (MH "Meals+") OR (MH "Food Preferences") OR (MH "Food Habits") OR (MH "Mediterranean Diet") OR (MH "Diet, Western") OR (MH "DASH Diet") OR (MH "Vegetarianism")

OR meal* OR "prudent diet" OR nordiet OR omniheart OR "Optimal Macronutrient Intake Trial to Prevent Heart Disease" OR ((Index OR score OR indices OR scoring) N3 (dietary OR diet OR food OR eating)) OR "adequacy index" OR kidmed OR MedDietScore

OR "dietary approaches to stop hypertension" OR "plant based diet" OR ((eating OR food* OR diet* OR calori*) N3 (pattern? OR habit? OR profile? OR recommendation? OR guideline? OR style* OR choice* OR intake OR quality))

OR

(dietary NEAR/1 change*) OR vegan* OR vegetarian* OR ((ethni* OR racial OR religio* OR asia* OR western OR Okinawa* OR adventist* OR 'mediterranean' OR Nordic* OR indo-mediterranean OR omni ${ }^{\star}$ ) N3 (calori* OR diet $^{\star}$ OR food OR eating))

OR (MH "Ethnic Groups+") AND ((mh diet) OR diet* OR (MH food) OR eat OR eating OR (MH "Eating") OR MH "food habits")) OR

((adhere* OR adhering) N3 (diet OR dietary OR food OR eat OR eating)) AND (guideline ${ }^{\star}$ OR guidance OR recommendation $\left.{ }^{\star}\right)$ )

(MH "Maternal Nutritional Physiology+") OR (MH "Maternal Exposure") OR (MH "Pregnancy+") OR (MH "Pregnancy in Adolescence+") OR (MH "Maternal Age 14 and Under") OR (MH "Pregnancy Outcomes") OR (MH "Mothers+") OR (MH "Prenatal Nutritional Physiology") OR (MH "Infant, Newborn+") OR (MH "Postnatal Period+") OR (MH "Periconceptual Period")

AND

(MH "Hypertension+") OR (MH "Nausea and Vomiting+") OR (MH "Vomiting+") OR (MH "Birth Weight") OR (MH "Glucose Tolerance Test") OR (MH "Prediabetic State") OR (MH "Glucose Intolerance") OR (MH "Insulin Resistance+") OR (MH "Blood Pressure+") OR (MH "Proteinuria+") OR (MH "Hemoglobin A, Glycosylated")

OR

(MH "Diabetes Mellitus, Gestational") OR (MH "Gestational Age") OR (MH "PreEclampsia+") OR (MH "Eclampsia+") OR (MH "Fetal Growth Retardation") OR (MH "Fetal Weight") OR (MH "Umbilical Arteries") OR (MH "Delivery, 
Obstetric+")

Limiters - Published Date: 19800101-; Peer Reviewed; English Language;

Exclude MEDLINE records; Pregnancy

Narrow by SubjectMajor: - energy intake

Narrow by SubjectMajor: - vegetarianism

Narrow by SubjectMajor: - women's health

Narrow by SubjectMajor: - pregnancy outcomes

Narrow by SubjectMajor: - pregnancy complications

Narrow by SubjectMajor: - food habits

Narrow by SubjectMajor: - diabetes mellitus, gestational

\section{Figure 2: Flow chart of literature search and screening results}

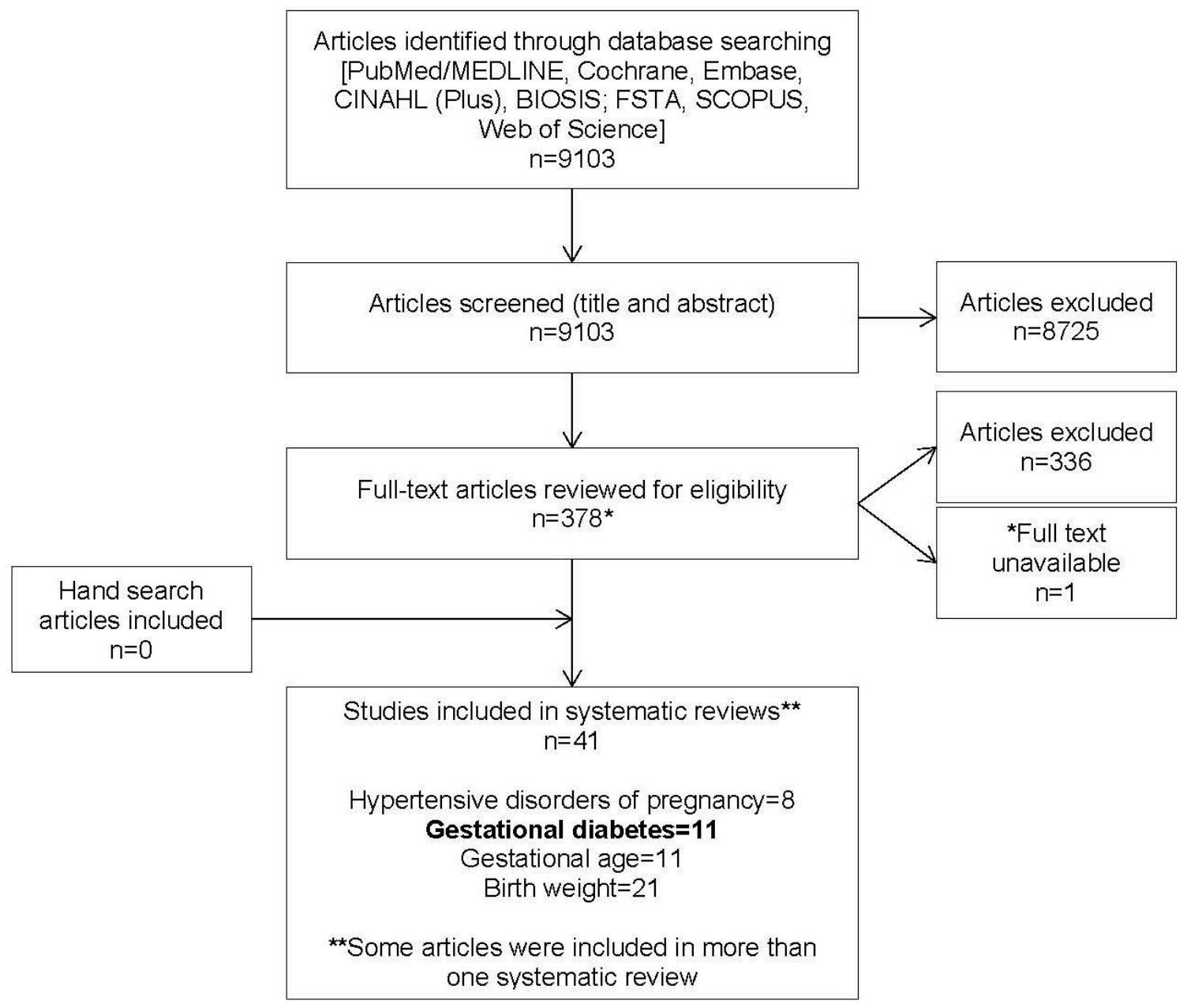




\section{Error! Reference source not found.}

The table below lists the excluded articles with at least one reason for exclusion, and may not reflect all possible reasons.

\section{Table 7. Excluded citations}

\section{Citation}

\section{Rationale}

1 Aaltonen, J, Ojala, T, Laitinen, K et al. Risk Reduction of Infant Insulin Resistance by Dietary Intervention during Pregnancy and Breastfeeding. Pediatric Academic Societies Annual Meeting; 2009 May 2 5; Baltimore MD, United States, 2009

Dependent variable

2 Abel, Ht, Bannert, N, Starke, I et al. Study into $\mathrm{Ca} / \mathrm{P}$ homeostasis in premature babies on different diets. Klin Padiatr, 1991, 203

3 Adami, G. F., Friedman, D., Cuneo, S. et al. Intravenous nutritional support in pregnancy. Independent Experience following biliopancreatic diversion. Clinical Nutrition, 1992, 11: 106-109

\section{Independent}

variable Akbari, Z., Mansourian, M., Kelishadi, R. Relationship of the intake of different food groups Independent by pregnant mothers with the birth weight and gestational age: Need for public and individual variable educational programs. J Educ Health Promot, 2015, 4. PMID:25883993.

5 Alfonso, H. Preventing preeclampsia: the evidence on nutrients. Nurs Womens Health, 2009, Study design 13: 419-21. PMID:19821918.

6 Ali, H. I., Jarrar, A. H., El Sadig, M. et al. Diet and carbohydrate food knowledge of multi- Study design ethnic women: a comparative analysis of pregnant women with and without Gestational Diabetes Mellitus. PLoS One, 2013, 8. PMID:24069200.

7 Alwan, N. A., Greenwood, D. C., Simpson, N. A. et al. Dietary iron intake during early Independent pregnancy and birth outcomes in a cohort of British women. Hum Reprod, 2011, 26: 911-9. variable PMID:21303776.

8 Andreasyan, K., Ponsonby, A. L., Dwyer, T. et al. Higher maternal dietary protein intake in Independent late pregnancy is associated with a lower infant ponderal index at birth. Eur J Clin Nutr, variable 2007, 61: 498-508. PMID:17136041.

$9 \quad$ Arkkola, T., Uusitalo, U., Kronberg-Kippila, C. et al. Seven distinct dietary patterns identified Dependent variable among pregnant Finnish women--associations with nutrient intake and sociodemographic factors. Public Health Nutr, 2008, 11: 176-82. PMID:17610760. 


\section{Citation}

10 Asaka, A., Imaizumi, Y., Inouye, E. Analysis of multiple births in Japan. V. Effects of gestational age, maternal age and other factors on growth rate of weight in twins. Jinrui Idengaku Zasshi, 1981, 26: 83-90. PMID:7328851.

11 Asbee, Sm, Jenkins, Tr, Butler, Jr et al. Dietary counseling prevents excessive weight gain during pregnancy, a randomized controlled trial. Obstet Gynecol, 2008, 111

12 Asp, N. G. Nutrition and human development. Scandinavian Journal of Food and Nutrition, 2006, 50

\section{Rationale}

Independent variable

Dependent variable

Independent variable, study design

13 Babson, Sg, Bramhall, Jl. Diet and growth in the premature infant. Journal of Pediatrics, 1969, 74: 890-900

14 Bakouei, S., Reisian, F., Lamyian, M. et al. High Intake of Manganese During Second Trimester, Increases the Risk of Preterm Delivery: A Large Scale Cohort Study. Glob J Health Sci, 2015, 7: 226-32. PMID:26156900.

15 Bao, W., Bowers, K., Tobias, D. K. et al. Prepregnancy low-carbohydrate dietary pattern and risk of gestational diabetes mellitus: a prospective cohort study. Am J Clin Nutr, 2014, 99: 1378-84. PMID:24717341.

16 Bao, W., Li, S., Chavarro, J. E. et al. Low Carbohydrate-Diet Scores and Long-term Risk of Dependent variable Type 2 Diabetes Among Women With a History of Gestational Diabetes Mellitus: A Prospective Cohort Study. Diabetes Care, 2016, 39: 43-9. PMID:26577416.

17 Bao, W., Tobias, D. K., Hu, F. B. et al. Pre-pregnancy potato consumption and risk of gestational diabetes mellitus: prospective cohort study. Bmj, 2016, 352. PMID:26759275.

Independent variable

18 Bao, W., Tobias, D. K., Olsen, S. F. et al. Pre-pregnancy fried food consumption and the risk Independent of gestational diabetes mellitus: a prospective cohort study. Diabetologia, 2014, 57: 2485-91. variable PMID:25303998.

19 Baron, R., Te Velde, S. J., Heymans, M. W. et al. The Relationships of Health Behaviour Independent and Psychological Characteristics with Spontaneous Preterm Birth in Nulliparous Women. variable Matern Child Health J, 2016, . PMID:27581004.

20 Bell, E. H., Geyer, J., Jones, L. A structured intervention improves breastfeeding success for Dependent variable ill or preterm infants. MCN Am J Matern Child Nurs, 1995, 20: 309-14. PMID:8551932.

21 Berntorp, K. E. Gestational diabetes: what's up?. Diabetologia, 2016, 59: 1382-1384

Study design 


\section{Citation}

22 Bertolotto, A., Volpe, L., Calianno, A. et al. Physical activity and dietary habits during pregnancy: effects on glucose tolerance. J Matern Fetal Neonatal Med, 2010, 23: 1310-4. PMID:20334531.

23 Bhatia, B. D., Banerjee, D., Agarwal, D. K. et al. Fetal growth: relationship with maternal dietary intakes. Indian J Pediatr, 1983, 50: 113-20. PMID:6618569.

24 Bjerregaard, P., Hansen, J. C. Effects of smoking and marine diet on birthweight in Greenland. Arctic Med Res, 1996, 55: 156-64. PMID:9115541.

\section{Rationale}

Independent variable, study design

Country

25 Bloomfield, F. H., Oliver, M. H., Hawkins, P. et al. A periconceptional nutritional origin for noninfectious preterm birth. Science, 2003, 300. PMID:12714735.

26 Bo, S., Rosato, R., Ciccone, G. et al. Simple lifestyle recommendations and the outcomes of gestational diabetes. A 2 × 2 factorial randomized trial. Diabetes Obes Metab, 2014, 16: 1032-5. PMID:24646172.

Independent variable

\section{Independent} variable, health status

27 Bobinski, R., Mikulska, M., Mojska, H. et al. Assessment of the diet components of pregnant women as predictors of risk of preterm birth and born baby with low birth weight. Ginekol Pol, 2015, 86: 292-9. PMID:26117989.

Independent variable, health status

Independent variable, study design

28 Bobinski, R., Mikulska, M., Mojska, H. et al. The Dietary Composition of Women Who Delivered Healthy Full-Term Infants, Preterm Infants, and Full-Term Infants Who Were Small for Gestational Age. Biol Res Nurs, 2015, 17: 495-502. PMID:25358685.

Independent variable, study design

29 Borberg, C., Gillmer, M. D., Brunner, E. J. et al. Obesity in pregnancy: the effect of dietary advice. Diabetes Care, 1980, 3: 476-81. PMID:6993162.

Independent variable

30 Borgen, I., Aamodt, G., Harsem, N. et al. Maternal sugar consumption and risk of preeclampsia in nulliparous Norwegian women. Eur J Clin Nutr, 2012, 66: 920-5. PMID:22713766.

31 Bower, D. The influence of dietary salt intake on pre-eclampsia. Journal of obstetrics and gynaecology of the British Commonwealth, 1961, 63: 123-6

32 Bowers, K., Tobias, D. K., Yeung, E. et al. A prospective study of prepregnancy dietary fat intake and risk of gestational diabetes. Am J Clin Nutr, 2012, 95: 446-53. PMID:22218158.

Independent variable

\section{Date}

Independent variable 


\section{Citation}

Rationale

33 BrantsA'ter, A. L., Haugen, M., Myhre, R. et al. Diet matters, particularly in pregnancy â€“ Results from MoBa studies of maternal diet and pregnancy outcomes. Norsk Epidemiologi, 2014, 24: 63-77

34 Brantsaeter, A. L., Myhre, R., Haugen, M. et al. Intake of probiotic food and risk of preeclampsia in primiparous women: the Norwegian Mother and Child Cohort Study. Am J Epidemiol, 2011, 174: 807-15. PMID:21821542.

35 Breslow, S, Belafsky, Ha, Shangold, Je et al. Control of weight gain in pregnancy: double blind study of a dieting aid. Clinical medicine, 1963, 70: 931-8

36 Brooke, O. G. Low birth weight babies. Nutrition and feeding. Br J Hosp Med, 1982, 28: 462- Dependent variable 9. PMID:7171896.

37 Brooke, O. G. Nutrition in the preterm infant. Lancet, 1983, 1: 514-6. PMID:6131220. Study subjects

38 Brown, J. E., Kahn, E. S., Hartman, T. J. Profet, profits, and proof: do nausea and vomiting of early pregnancy protect women from harmful vegetables?. Am J Obstet Gynecol, 1997, 176: 179-81. PMID:9024110.

39 Brumfield, C. G., Huddleston, J. F. The management of diabetic ketoacidosis in pregnancy. Clin Obstet Gynecol, 1984, 27: 50-9. PMID:6423330.

40 Bruno, R., Petrella, E., Bertarini, V. et al. Adherence to a lifestyle programme in overweight/obese pregnant women and effect on gestational diabetes mellitus: a randomized controlled trial. Matern Child Nutr, 2016, . PMID:27647837.

\section{Date}

Independent

variable

Independent variable

Independent variable, study design

41 Buchanan, T. A., Kjos, S. L. Diabetes and pregnancy. Curr Ther Endocrinol Metab, 1994, 5: Study design 278-83. PMID:7704732.

42 Buul, E, Rijpkema, A, Steegers, E et al. Chronic dietary sodium restriction in pregnancy Independent reduces calcium intake. J Perinat Med, 1992, 20

variable

43 Campbell, Dm. Dietary restriction in obesity and its effect on neonatal outcome. Nutrition in Pregnancy. Proceedings of 10th Study Group of the Rcog; 1983; London, UK, 1983, : 243-50

44 Canda, M. T., Sezer, O., Demir, N. An audit of seafood consumption awareness during pregnancy and its association with maternal and fetal outcomes in a Turkish population. $J$

Not peer-reviewed Obstet Gynaecol, 2011, 31: 293-7. PMID:21534748. 


\section{Citation}

Rationale

45 Carmichael, S. L., Yang, W., Shaw, G. M. Maternal dietary nutrient intake and risk of preterm delivery. Am J Perinatol, 2013, 30: 579-88. PMID:23208764.

Independent variable, study design

46 Carter, J. P., Furman, T., Hutcheson, H. R. Preeclampsia and reproductive performance in a community of vegans. South Med J, 1987, 80: 692-7. PMID:3589760.

Independent variable

47 Carver, Jd, Saste, Md, Sosa, R et al. Dietary nucleotide (NT) effects on superior mesenteric artery (SMA) blood flow in preterm infants. Pediatr Res, 2000, 47

Independent variable

48 C'De Baca, J., Lapham, S. C., Skipper, B. J. et al. Use of computer interview data to test associations between risk factors and pregnancy outcomes. Comput Biomed Res, 1997, 30: 232-43. PMID:9281330.

49 Chamberlain, G. Epidemiology and aetiology of the preterm baby. Clin Obstet Gynaecol, 1984, 11: 297-314. PMID:6478726.

50 Chandler-Laney, P. C., Schneider, C. R., Gower, B. A. et al. Association of late-night carbohydrate intake with glucose tolerance among pregnant African American women. Matern Child Nutr, 2016, 12: 688-98. PMID:25786515.

51 Chavarro, J. E., Halldorsson, T. I., Leth, T. et al. A prospective study of trans fat intake and risk of preeclampsia in Denmark. Eur J Clin Nutr, 2011, 65: 944-51. PMID:21559043.

Independent variable

\section{Study design}

Independent

variable

Independent

variable

52 Chen, C. M., Weng, H. C., Li, Y. C. et al. The evaluation of dietary intervention on the blood Language glucose level of gestational diabetes mellitus pregnant women. Nutritional Sciences Journal, 1999, 24: 250-261

53 Chen, L., Hu, F. B., Yeung, E. et al. Prospective study of pre-gravid sugar-sweetened beverage consumption and the risk of gestational diabetes mellitus. Diabetes Care, 2009, 32: 2236-41. PMID:19940226.

54 Chong, M. F., Chia, A. R., Colega, M. et al. Maternal Protein Intake during Pregnancy Is Not Independent Associated with Offspring Birth Weight in a Multiethnic Asian Population. J Nutr, 2015, 145: variable 1303-10. PMID:25948786. 


\section{Citation}

Rationale

55 Christian, K, Andreas, M, Martin, F. Diet and lifestyle modification in mothers with burnout syndrome: Ayurvedic versus conventional standard counselling-design of a randomised clinical pilot study (VEDA-Trial) [abstract]. European journal of integrative medicine [abstracts of the 5th european congress for integrative medicine; 2012 sept 21-22; flo, 2012, 4: 47-8

56 Clapp, J. F. Effects of Diet and Exercise on Insulin Resistance during Pregnancy. Metab Syndr Relat Disord, 2006, 4: 84-90. PMID:18370754.

57 Clausen, T., Slott, M., Solvoll, K. et al. High intake of energy, sucrose, and polyunsaturated fatty acids is associated with increased risk of preeclampsia. Am J Obstet Gynecol, 2001, 185: 451-8. PMID:11518908.

58 Coelho Nde, L., Cunha, D. B., Esteves, A. P. et al. Dietary patterns in pregnancy and birth Study design weight. Rev Saude Publica, 2015, 49. PMID:26398873.

59 Cooney, G. Food for thought. Midwives, 2008, 11: 30-1. PMID:24902215.

Study design

60 Cooper, M. L. Stories to learn from: toxemia in pregnancy. Midwifery Today Int Midwife, 2014, : 18-21. PMID:25980103.

61 Corbett, M. A., Burst, H. V. Nutritional intervention in pregnancy. J Nurse Midwifery, 1983, 28: 23-9. PMID:6554311.

62 Cosgrove, M., Davies, D. P. Poor diet in pregnancy may be a proxy for some other hostile influence on fetal growth [8]. Br Med J, 1996, 312: 1478-1479

Not peer-reviewed

Study design, independent variable

Independent variable, study design

63 Costa-Orvay, Ja, Figueras-Aloy, J, Romera, G et al. The effects of varying protein and energy intakes on the growth and body composition of very low birth weight infants. Nutr $\mathrm{J}$, 2011, 10

64 Crozier, S. R., Inskip, H. M., Godfrey, K. M. et al. Nausea and vomiting in early pregnancy: Effects on food intake and diet quality. Matern Child Nutr, 2016, . PMID:27896913.

65 Dancause, K. N., Mutran, D., Elgbeili, G. et al. Dietary change mediates relationships between stress during pregnancy and infant head circumference measures: the QF2011 study. Matern Child Nutr, 2016, . PMID:27562643.

Independent variable

Dependent variable Independent variable 


\section{Citation}

66 Darling, A. M., Mitchell, A. A., Werler, M. M. Preconceptional Iron Intake and Gestational Diabetes Mellitus. Int J Environ Res Public Health, 2016, 13. PMID:27231921.

\section{Rationale}

Independent variable

67 Davidson, J. K. Newer approaches to diet management of diabetes: calorie control. Med Study design Times, 1980, 108: 35-40. PMID:7374404.

68 Davies, W. E., Hopkins, P. C., Rose, S. J. et al. The influence of different taurine diets on Independent hearing development in normal babies. A preliminary report. Adv Exp Med Biol, 1996, 403: variable 631-7. PMID:8915404.

69 Davison, J. M., Lindheimer, M. D. Pregnancy in renal transplant recipients. J Reprod Med, Health status 1982, 27: 613-21. PMID:6757420.

70 Dawn, Cs. Effects of substandard prenatal diet and nutrition on the development and

71 de Seymour, J., Chia, A., Colega, M. et al. Maternal Dietary Patterns and Gestational

72 Deka, D., Sharma, N. Nutrition in pregnancy and lactation. Perinatology, 2005, 7: 1-15

73 Delemarre, F. M., van Leest, L. A., Jongsma, H. W. et al. Effect of low-sodium diet on

74 Demmelmair, $\mathrm{H}$, Klingler, M, Campoy, $\mathrm{C}$ et al. The influence of habitual diet and increased

75 Deveer, R., Deveer, M., Akbaba, E. et al. The effect of diet on pregnancy outcomes among

76 Dieckmann, Wj, Davis, Me, Rynkiewicz, Lm et al. Does the administration of

77 Diet \& nutrition. Good news: caffeine in pregnancy doesn't affect the baby's growth...and folic incidence of pre-eclampsia of pregnancy. J Obstet Gynaecol India, 1961, 12: 237-45 Diabetes Mellitus in a Multi-Ethnic Asian Cohort: The GUSTO Study. Nutrients, 2016, 8. PMID:27657116. uteroplacental circulation. J Matern Fetal Med, 2000, 9: 197-200. PMID:11048827. docosahexaenoic acid intake during pregnancy on the fatty acid composition of individual placental lipids [Study design]. J Pediatr Gastroenterol Nutr, 2005, 40: 622-3 pregnant with abnormal glucose challenge test. Eur Rev Med Pharmacol Sci, 2013, 17: 1258-61. PMID:23690197. diethylstilbestrol during pregnancy have therapeutic value?. Am J Obstet Gynecol, 1953, 66: $1062-75$

acid seems to prevent cleft lip. Child Health Alert, 2007, 25: 5-6. PMID:17443983.

\section{Date}

Study design Study design Independent variable

\section{Study design}

Independent

variable

\section{Date}

Not peer-reviewed 


\section{Citation}

78 Dodd, J. M., Deussen, A. R., Mohamad, I. et al. The effect of antenatal lifestyle advice for women who are overweight or obese on secondary measures of neonatal body composition The LIMIT randomised trial. BJOG: An International Journal of Obstetrics and Gynaecology, 2016, 123: 244-253

79 Dodd, J. M., McPhee, A. J., Turnbull, D. et al. The effects of antenatal dietary and lifestyle advice for women who are overweight or obese on neonatal health outcomes: the LIMIT randomised trial. BMC Med, 2014, 12. PMID:25315325.

80 Dominguez, L. J., Martinez-Gonzalez, M. A., Basterra-Gortari, F. J. et al. Fast food consumption and gestational diabetes incidence in the SUN project. PLoS One, 2014, 9. PMID:25215961.

81 Donnelly, J, Horan, M, Walsh, J et al. Impact of a Low GI Diet on Neonatal Body Composition [ROLO Kids]. Pediatric Academic Societies Annual Meeting, 2013,

82 Donnelly, J. M., Walsh, J. M., Byrne, J. et al. Impact of maternal diet on neonatal anthropometry: a randomized controlled trial. Pediatr Obes, 2015, 10: 52-6. PMID:24443392.

Rationale

Independent variable

Independent variable

\section{Independent}

variable

Not peer-reviewed

Independent

variable

Study design PMID:12630147.

84 Doyle, W., Crawford, M. A., Wynn, A. H. A. et al. Maternal nutrient intake and birth-weight. Journal of Human Nutrition and Dietetics, 1989, 2: 415-422

Independent

variable

85 Drake, A. J., McPherson, R. C., Godfrey, K. M. et al. An unbalanced maternal diet in pregnancy associates with offspring epigenetic changes in genes controlling glucocorticoid action and foetal growth. Clin Endocrinol (Oxf), 2012, 77: 808-15. PMID:22642564.

86 Drouillet, P., Kaminski, M., De Lauzon-Guillain, B. et al. Association between maternal seafood consumption before pregnancy and fetal growth: evidence for an association in overweight women. The EDEN mother-child cohort. Paediatr Perinat Epidemiol, 2009, 23: 76-86. PMID:19228317.

87 Dubois, S., Coulombe, C., Pencharz, P. et al. Ability of the Higgins Nutrition Intervention Program to improve adolescent pregnancy outcome. J Am Diet Assoc, 1997, 97: 871-8. PMID:9259709.

88 Dunn, C., Kolasa, K., Dunn, P. C. et al. Dietary intake of pregnant adolescents in a rural southern community. J Am Diet Assoc, 1994, 94: 1040-1. PMID:8071488.
Dependent variable

Independent

variable

Independent

variable

Independent variable, dependent variable 


\section{Citation}

\section{Rationale}

89 Ebbs, Jh, Tisdall, Ff, Scott, Wa. The influence of prenatal diet on the mother and child. Journal of Nutrition, 1941, 22: 515-26

90 Elmacioglu, F., Surucu, B., Alper, T. et al. Is adequate and balanced nutrition during pregnancy more effective than iron and folic acid supplements?. Central European Journal of Medicine, 2010, 5: 235-242

91 Ershoff, Dh, Aaronson, Nk, Danaher, Bg et al. Behavioral, health, and cost outcomes of an HMO based prenatal health education program. Public health reports, 1983, 98: 536-47

\section{Date}

Dependent variable

Independent

variable

92 Ershoff, D. H., Aaronson, N. K., Danaher, B. G. et al.. Behavioral, health, and cost outcomes Duplicate of an HMO-based prenatal health education program. Public Health Rep, 1983, 98: 536-47. PMID:6419268.

93 Eshriqui, I., Vilela, A. A., Rebelo, F. et al. Gestational dietary patterns are not associated with blood pressure changes during pregnancy and early postpartum in a Brazilian prospective cohort. Eur J Nutr, 2016, 55: 21-32. PMID:25526968.

94 Fairburn, C. G., Stein, A., Jones, R. Eating habits and eating disorders during pregnancy. Psychosom Med, 1992, 54: 665-72. PMID:1454960.

95 Farbu, J., Haugen, M., Meltzer, H. M. et al. Impact of singlehood during pregnancy on dietary intake and birth outcomes- a study in the Norwegian Mother and Child Cohort Study. BMC Pregnancy Childbirth, 2014, 14. PMID:25475509.

96 Fard, N Mehrabian F Sarraf-Zadegan NS. Fat-modified diets during pregnancy and lactation Country and serum lipids after birth. Indian J Pediatr, 2004, 71: 683-7

97 Farland, L. V., Rifas-Shiman, S. L., Gillman, M. W. Early Pregnancy Cravings, Dietary Intake, and Development of Abnormal Glucose Tolerance. J Acad Nutr Diet, 2015, 115. PMID:26099686.

98 Ferland, S., O'Brien, H. T. Maternal dietary intake and pregnancy outcome. J Reprod Med, 2003, 48: 86-94. PMID:12621791.

99 Flynn, A. C., Seed, P. T., Patel, N. et al. Dietary patterns in obese pregnant women; influence of a behavioral intervention of diet and physical activity in the UPBEAT randomized controlled trial. Int J Behav Nutr Phys Act, 2016, 13. PMID:27894316.

100 Ford, J. H. Preconception risk factors and SGA babies: Papilloma virus, omega 3 and fat soluble vitamin deficiencies. Early Hum Dev, 2011, 87: 785-9. PMID:21705161.
Dependent variable

Independent variable

\section{Independent}

variable

Study design, independent variable

\section{Independent}

variable

Health status

Independent

variable 


\section{Citation}

101 Fowles, E. R., Gabrielson, M. First trimester predictors of diet and birth outcomes in lowincome pregnant women. J Community Health Nurs, 2005, 22: 117-30. PMID:15877540.

102 Fraser, R. B., Ford, F. A., Milner, R. D. G. A controlled trial of a high dietary fibre intake in pregnancy-effects in plasma glucose and insulin levels. Diabetologia, 1983, 25: 238-241

103 Fraser, Rb. High fibre diets in pregnancy. Nutrition in Pregnancy. Proceedings of 10th Study Group of the Royal College of Obstetricians and Gynaecologists; 1982 September, 1983, : 269-80

104 Garratt, F. N. Pre-eclampsia: a challenge to public health teams worldwide to ensure that maternal diets contain adequate levels of folic acid, $n 3$ polyunsaturated fatty acids and vitamin D at conception. Public Health, 2009, 123: 95-6. PMID:19058819.

105 Gennaro, S., Biesecker, B., Fantasia, H. C. et al. Nutrition profiles of African [corrected] American women in the third trimester. MCN Am J Matern Child Nurs, 2011, 36: 120-6. PMID:21350375.

106 Gerrard, J., Popeski, D., Ebbeling, L. et al. Dietary omega 3 fatty acids and gestational hypertension in the Inuit. Arctic Med Res, 1991, : 763-7. PMID:1365294.

107 Gesteiro, E., Rodriguez Bernal, B., Bastida, S. et al. Maternal diets with low healthy eating index or Mediterranean diet adherence scores are associated with high cord-blood insulin levels and insulin resistance markers at birth. Eur J Clin Nutr, 2012, 66: 1008-15. PMID:22828732.

108 Ghebremeskel, K., Leighfield, M., Ashwell, M. et al. Infant brain lipids and diet [1]. Lancet, 1992, 340: 1093-1094

109 Gillen, L., Tapsell, L. C., Martin, G. S. et al. The type and frequency of consumption of carbohydrate-rich foods may play a role in the clinical expression of insulin resistance during pregnancy. Dietetics, 2002, 59: 135-143

110 Glueck, C. J., Goldenberg, N., Pranikoff, J. et al. Effects of metformin-diet intervention before and throughout pregnancy on obstetric and neonatal outcomes in patients with polycystic ovary syndrome. Curr Med Res Opin, 2013, 29: 55-62. PMID:23205605.

\section{Rationale}

Independent variable, study design

Independent variable

Independent variable, not peer reviewed

Study design

Independent variable, dependent variable

Independent variable, study design

Dependent variable

Dependent variable

Dependent variable

Health status 


\section{Citation}

Rationale

111 Godfrey, K., Robinson, S., Barker, D. J. et al. Maternal nutrition in early and late pregnancy in relation to placental and fetal growth. Bmj, 1996, 312: 410-4. PMID:8601112.

Independent variable

112 Grant, S. M., Wolever, T. M., O'Connor, D. L. et al. Effect of a low glycaemic index diet on Independent blood glucose in women with gestational hyperglycaemia. Diabetes Res Clin Pract, 2011, 91: variable 15-22. PMID:21094553.

113 Gray-Donald, K., Robinson, E., Collier, A. et al. Intervening to reduce weight gain in pregnancy and gestational diabetes mellitus in Cree communities: an evaluation. Cmaj, 2000, 163: 1247-51. PMID:11107459.

114 Grivell, R. M., Yelland, L. N., Deussen, A. et al. Antenatal dietary and lifestyle advice for women who are overweight or obese and the effect on fetal growth and adiposity: the LIMIT randomised trial. Bjog, 2016, 123: 233-43. PMID:26841216.

115 Guilloty, N. I., Soto, R., Anzalota, L. et al. Diet, Pre-pregnancy BMI, and Gestational Weight Independent Gain in Puerto Rican Women. Matern Child Health J, 2015, 19: 2453-61. PMID:26100133. variable, dependent variable

116 Guldner, L., Monfort, C., Rouget, F. et al. Maternal fish and shellfish intake and pregnancy outcomes: a prospective cohort study in Brittany, France. Environ Health, 2007, 6. PMID:17958907.

117 Gupta, A. P., Bhandari, B., Gupta, A. et al. Stool pH and sugar in preterm neonates. Indian J Pediatr, 1984, 51: 391-3. PMID:6526446.

\section{Independent}

variable

\section{Independent} variable

118 Haas, A. V. Diet du jour! Pregnancy and popular diets. Midwifery Today Int Midwife, 2014, : 53-5. PMID:25975083.

119 Haggarty, P., Campbell, D. M., Duthie, S. et al. Diet and deprivation in pregnancy. Br J Nutr, 2009, 102: 1487-97. PMID:19682400.

120 Halldorsson, T. I., Thorsdottir, I., Meltzer, H. M. et al. Dioxin-like activity in plasma among Danish pregnant women: dietary predictors, birth weight and infant development. Environ Res, 2009, 109: 22-8. PMID:18945425.

121 Halldorsson, T. I., Thorsdottir, I., Meltzer, H. M. et al. Linking exposure to polychlorinated biphenyls with fatty fish consumption and reduced fetal growth among Danish pregnant women: a cause for concern?. Am J Epidemiol, 2008, 168: 958-65. PMID:18718897.
Study design, non peer-reviewed

Independent variable

Independent variable

Independent variable 


\section{Citation}

122 Hankin, Me, Symonds, Em. Body weight, diet and pre-eclamptic toxaemia of pregnancy. gynaecology, 1962, 4: 156-60

123 Harper, V., Maclnnes, R., Campbell, D. et al. Increased birth weight in northerly islands: is fish consumption a red herring?. Bmj, 1991, 303. PMID:1878642.

\section{Rationale}

Date

Independent

variable

124 Hatfield, Hm, Dunstan, Ja, Hayes, L et al. Dietary N-3 polyunsaturated fatty acid (PUFA) supplementation during pregnancy is associated with changes in cord blood (CB) progenitor numbers and responsiveness to IL-5 in infants at risk of atopy [Abstract]. Journal of allergy and clinical immunology, 2003, 111

125 Haugen, M., Brantsaeter, A. L., Trogstad, L. et al. Vitamin D supplementation and reduced risk of preeclampsia in nulliparous women. Epidemiology, 2009, 20: 720-6. PMID:19451820.

Study design

Independent

variable

126 Hayashi, Tt, Phitaksphraiwan, P, Willson, Jr. Effects of diet and diuretic agents in pregnancy Date toxemias. Obstet Gynecol, 1963, 22: 327-34

127 Healthy diet halves the risk of diabetes after pregnancy. Kidney Care, 2013, 10: 6-6 Dependent variable

128 Hegsted, D. M. What is a healthful diet?. Prim Care, 1982, 9: 445-73. PMID:6924383. Dependent variable

129 Heim, T. Energy and lipid requirements of the fetus and the preterm infant. J Pediatr Gastroenterol Nutr, 1983, . PMID:6417303.

Independent variable, study design

130 Hellmuth, C., Lindsay, K. L., Uhl, O. et al. Association of maternal prepregnancy BMI with metabolomic profile across gestation. Int J Obes (Lond), 2017, 41: 159-169. PMID:27569686.

131 Hennessy, M. D., Volpe, S. L., Sammel, M. D. et al. Skipping meals and less walking among African Americans diagnosed with preterm labor. J Nurs Scholarsh, 2010, 42: 147-55. PMID:20618599.

132 Heppe, D. H., Steegers, E. A., Timmermans, S. et al. Maternal fish consumption, fetal growth and the risks of neonatal complications: the Generation R Study. Br J Nutr, 2011, 105: 938-49. PMID:21266095.

133 Hernandez-Diaz, S., Boeke, C. E., Romans, A. T. et al. Triggers of spontaneous preterm delivery--why today?. Paediatr Perinat Epidemiol, 2014, 28: 79-87. PMID:24384058.

Dependent variable

\section{Independent}

variable

Independent variable 


\section{Citation}

134 Herrera, Mg, Mora, Jo, Paredes, B et al. Maternal weight/height and the effect of food supplementation during pregnancy and lactation. Maternal Nutrition during Pregnancy and Lactation. A Nestle Foundation Workshop; 1979 April 26-27; Lausanne Switzerland, 1980, : 252-63

135 Hoff, C., Wertelecki, W., Reyes, E. et al. Diet, blood pressure, and hematologic variables of nulliparous women attending a prenatal clinic. Obstet Gynecol, 1986, 67: 868-72. PMID:3703412.

136 Hoffman, D, Uauy, R, Birch, D et al. Essentiality of dietary docosahexaenoic acid (dha) for optimal visual maturation in preterm infants: plasma and red blood cell (rbc) fatty acid profiles. lovs, 1992, 33

137 Hoffman, Dr, Uauy, R. Essentiality of dietary n-3 fatty acids for premature infants; plasma and red blood cell fatty acid composition. Lipids, 1992, 27: 886-95

138 Hollingsworth, D. R., Ney, D., Stubblefield, N. et al. Metabolic and therapeutic assessment of gestational diabetes by two-hour and twenty-four-hour isocaloric meal tolerance tests. Diabetes, 1985, : 81-7. PMID:3888746.

139 Hook, E. B. Influence of pregnancy on dietary selection. Int J Obes, 1980, 4: 338-40. PMID:7419353.

140 Horan, M. K., McGowan, C. A., Gibney, E. R. et al. Maternal low glycaemic index diet, fat intake and postprandial glucose influences neonatal adiposity--secondary analysis from the ROLO study. Nutr J, 2014, 13. PMID:25084967.

141 Horan, M. K., McGowan, C. A., Gibney, E. R. et al. Maternal Nutrition and Glycaemic Index during Pregnancy Impacts on Offspring Adiposity at 6 Months of Age--Analysis from the ROLO Randomised Controlled Trial. Nutrients, 2016, 8. PMID:26742066.

142 Horan, M. K., McGowan, C. A., Gibney, E. R. et al. Maternal nutrition and glycaemic index during pregnancy impacts on offspring adiposity at 6 months of ageâ $€$ "analysis from the ROLO randomised controlled trial. Nutrients, 2016, 8.

143 Huh, S. Y., Rifas-Shiman, S. L., Kleinman, K. P. et al. Maternal protein intake is not associated with infant blood pressure. Int J Epidemiol, 2005, 34: 378-84. PMID:15576466.

144 Hui, A. L., Ludwig, S. M., Gardiner, P. et al. Community-based exercise and dietary intervention during pregnancy: A pilot study. Canadian Journal of Diabetes, 2006, 30: 169175

\section{Rationale}

Independent variable, not peer

reviewed

Independent

variable

Dependent variable

Independent

variable

Dependent variable

Study design

Independent

variable

Independent variable, dependent variable

Duplicate Independent variable

\section{Independent} variable 


\section{Citation}

145 Hui, A., Back, L., Ludwig, S. et al. Lifestyle intervention on diet and exercise reduced excessive gestational weight gain in pregnant women under a randomized controlled trial. Obstetrical and Gynecological Survey, 2012, 67: 263-264

146 lyengar, L. Effects of dietary supplements late in pregnancy on the expectant mother and her Date newborn. Indian Journal of Medical Research, 1967, 55: 85-9

147 Jedrychowski, W., Perera, F., Mrozek-Budzyn, D. et al. Higher fish consumption in pregnancy may confer protection against the harmful effect of prenatal exposure to fine particulate matter. Ann Nutr Metab, 2010, 56: 119-26. PMID:20134157.

148 Jing, W., Huang, Y., Liu, X. et al. The effect of a personalized intervention on weight gain and physical activity among pregnant women in China. Int J Gynaecol Obstet, 2015, 129: 138-41. PMID:25697965.

149 Johnson, A. A., Knight, E. M., Edwards, C. H. et al. Dietary intakes, anthropometric measurements and pregnancy outcomes. J Nutr, 1994, 124. PMID:8201444.

\section{Rationale}

Independent

variable

Independent

variable

Independent

variable

Independent

variable

150 Jovanovic-Peterson, L, Durak, Ep, Peterson, Cm. Randomized trial of diet vs diet plus

Health status cardiovascular conditioning on glucose levels in gestational diabetes. Am J Obstet Gynecol, 1989, 161: 415-9

151 Jovanovic-Peterson, L., Peterson, C. M. Turning point in the management of pregnancies complicated by diabetes. Normoglycemia with self blood glucose monitoring of diet and insulin dosing. ASAIO Trans, 1990, 36: 799-804. PMID:2268482.

Independent variable, study design

152 Jowett, N. I., Nichol, S. G. Diabetic pregnancy. Midwives Chron, 1987, 100: 33-6. PMID:3645266.

153 Kafatos, A. G., Vlachonikolis, I. G., Codrington, C. A. Nutrition during pregnancy: the effects of an educational intervention program in Greece. Am J Clin Nutr, 1989, 50: 970-9.

Study design PMID:2816804.

154 Kalhan, S. C., Tserng, K. Y., Gilfillan, C. et al. Metabolism of urea and glucose in normal and diabetic pregnancy. Metabolism, 1982, 31: 824-33. PMID:7098852.

155 Kaseb, F., Kimiagar, M., Ghafarpoor, M. et al. Effect of traditional food supplementation during pregnancy on maternal weight gain and birthweight. Int J Vitam Nutr Res, 2002, 72: 389-93. PMID:12596505.

Independent variable

\section{Dependent variable}

Independent variable 


\section{Citation}

Rationale

156 Kelleher, C. C., Viljoen, K., Khalii, H. et al. Longitudinal follow-up of the relationship between

Study design dietary intake and growth and development in the Lifeways cross-generation cohort study 2001-2013. Proc Nutr Soc, 2014, 73: 118-31. PMID:24300176.

157 Kesmodel, U., Olsen, S. F., Salvig, J. D. Marine n-3 fatty acid and calcium intake in relation to pregnancy induced hypertension, intrauterine growth retardation, and preterm delivery. A case-control study. Acta Obstet Gynecol Scand, 1997, 76: 38-44. PMID:9033242.

158 Khoury, J, Haugen, G, Tonstad, S et al. Effect of an antiatherogenic diet on maternal and fetal Doppler velocimetry: a randomized clinical trial. 35th Nordic Congress of Obstetrics and Gynecology; 2006 May 23-25; Goteburg, Sweden, 2008,

159 Khoury, J, Henriksen, T, Seljeflot, I et al. Effects of a cholesterol-lowering diet during pregnancy on cardiovascular risk factors and pregnany outcome: a randomized clinical trial [Study design]. Atherosclerosis. Supplements, 2006, 7

160 Khoury, J., Haugen, G., Tonstad, S. et al. Effect of a cholesterol-lowering diet during pregnancy on maternal and fetal Doppler velocimetry: the CARRDIP study. Am J Obstet Gynecol, 2007, 196. PMID:17547890.

161 Kinnunen, T. I., Pasanen, M., Aittasalo, M. et al. Preventing excessive weight gain during pregnancy - a controlled trial in primary health care. Eur J Clin Nutr, 2007, 61: 884-91. PMID:17228348.

162 Kinnunen, T. I., Puhkala, J., Raitanen, J. et al. Effects of dietary counselling on food habits and dietary intake of Finnish pregnant women at increased risk for gestational diabetes - a secondary analysis of a cluster-randomized controlled trial. Matern Child Nutr, 2014, 10: 18497. PMID:22735030.

163 Kizirian, N. V., Kong, Y., Muirhead, R. et al. Effects of a low-glycemic index diet during pregnancy on offspring growth, body composition, and vascular health: a pilot randomized controlled trial. Am J Clin Nutr, 2016, 103: 1073-82. PMID:26936333.

164 Klebanoff, M. A., Harper, M., Lai, Y. et al. Fish consumption, erythrocyte fatty acids, and preterm birth. Obstet Gynecol, 2011, 117: 1071-7. PMID:21508745.

Independent variable

Not peer-reviewed

Study design

\section{Independent}

variable

\section{Independent}

variable

\section{Independent}

variable, dependent

variable

Independent

variable

Independent

variable

\section{Independent}

variable

during pregnancy and gestational weight gain, birth weight and postpartum weight retention: a study within the Danish National Birth Cohort. Br J Nutr, 2013, 109: 1471-8. PMID:22906835. 


\section{Citation}

166 Knuist, M., Bonsel, G. J., Zondervan, H. A. et al. Low sodium diet and pregnancy-induced hypertension: a multi-centre randomised controlled trial. Br J Obstet Gynaecol, 1998, 105: 430-4. PMID:9609271.

167 Koivusalo, S. B., Rono, K., Klemetti, M. M. et al. Gestational Diabetes Mellitus Can Be Prevented by Lifestyle Intervention: The Finnish Gestational Diabetes Prevention Study (RADIEL): A Randomized Controlled Trial. Diabetes Care, 2016, 39: 24-30. PMID:26223239.

168 Kokanali, M. K., Tokmak, A., Kaymak, O. et al. The effect of treatment on pregnancy outcomes in women with one elevated oral glucose tolerance test value. Ginekol Pol, 2014, 85: 748-53. PMID:25546925.

169 Kolu, P., Raitanen, J., Rissanen, P. et al. Cost-effectiveness of lifestyle counselling as primary prevention of gestational diabetes mellitus: findings from a cluster-randomised trial. PLoS One, 2013, 8. PMID:23457562.

170 Korpi-Hyovalti, E., Schwab, U., Laaksonen, D. E. et al. Effect of intensive counselling on the quality of dietary fats in pregnant women at high risk of gestational diabetes mellitus. Br J Nutr, 2012, 108: 910-7. PMID:22093485.

171 Kubota, K., Itoh, H., Tasaka, M. et al. Changes of maternal dietary intake, bodyweight and fetal growth throughout pregnancy in pregnant Japanese women. J Obstet Gynaecol Res, 2013, 39: 1383-90. PMID:23815608.

172 Kumar, P., Nangia, S., Saili, A. et al. Growth and morbidity patterns of exclusively breast-fed Dependent variable preterm babies. Indian Pediatr, 1999, 36: 296-300. PMID:10713842.

173 Lakin, V., Haggarty, P., Abramovich, D. R. et al. Dietary intake and tissue concentration of fatty acids in omnivore, vegetarian and diabetic pregnancy. Prostaglandins Leukot Essent Fatty Acids, 1998, 59: 209-20. PMID:9844995.

174 Langley-Evans, A. J., Langley-Evans, S. C. Relationship between maternal nutrient intakes in early and late pregnancy and infants weight and proportions at birth: prospective cohort study. J R Soc Promot Health, 2003, 123: 210-6. PMID:14669495.

175 Laraia, B. A., Siega-Riz, A. M., Kaufman, J. S. et al. Proximity of supermarkets is positively Dependent variable associated with diet quality index for pregnancy. Prev Med, 2004, 39: 869-75. PMID:15475018.

176 Latva-Pukkila, U., Isolauri, E., Laitinen, K. Dietary and clinical impacts of nausea and vomiting during pregnancy. J Hum Nutr Diet, 2010, 23: 69-77. PMID:19943842.

\section{Rationale}

Independent variable

Independent variable

\section{Independent}

variable

Independent

variable

\section{Independent}

variable

Independent

variable

Independent

variable

\section{Independent}

variable

Dependent variable 


\section{Citation}

Rationale

177 Leblance, H., Passa, P. Diabetes and pregnancy. Revue du Praticien - Medecine Generale, 1992, 6: 577-582

178 Lechtig, A, Habicht, Jp, Delgado, $\mathrm{H}$ et al. Effect of food supplementation during pregnancy on birthweight. Pediatrics, 1975, 56: 508-20

179 Lenders, C. M., Hediger, M. L., Scholl, T. O. et al. Gestational age and infant size at birth are associated with dietary sugar intake among pregnant adolescents. J Nutr, 1997, 127: 1113-7. PMID:9187625.

180 Ley, S. H., Hanley, A. J., Retnakaran, R. et al. Effect of macronutrient intake during the second trimester on glucose metabolism later in pregnancy. Am J Clin Nutr, 2011, 94: 123240. PMID:21955650.

Study design, language

Date Independent variable

Independent variable, study design

181 L'Heureux, J. Got sugar? Tips on preventing diabetes. Posit Living, 2002, 11: 12-4. PMID:12083048.

182 Li, S., Zhu, Y., Chavarro, J. E. et al. Healthful Dietary Patterns and the Risk of Hypertension Among Women With a History of Gestational Diabetes Mellitus: A Prospective Cohort Study. Hypertension, 2016, 67: 1157-65. PMID:27091899.

183 Lilja, G, Dannaeus, A, Foucard, T et al. Effects of maternal diet during late pregnancy and lactation on the development of atopic disease in infants up to 18 months of age - in-vivo results. Clinical and Experimental Allergy, 1989, 19: 473-9

184 Liu, X., Lv, L., Zhang, H. et al. Folic acid supplementation, dietary folate intake and risk of preterm birth in China. European Journal of Nutrition, 2016, 55: 1411-1422

Study design

Dependent variable

Dependent variable

Independent variable, study design

185 Lorber, D. Gestational diabetes: The hidden epidemic. Female Patient - Practical Ob/Gyn Study design Medicine, 1990, 15: 15-25

186 Luoto, R, Nermes, M, Laitinen, K et al. Impact of Maternal Probiotic-Supplemented Dietary Counselling on Pregnancy Outcome and Prenatal and Postnatal Growth: A Double-Blind, Placebo-Controlled Study. Pediatric Academic Societies Annual Meeting; 2009 May 2 5; Baltimore MD, United States, 2009,

187 MacGillivray, I. Aetiology of pre-eclampsia. Br J Hosp Med, 1981, 26. PMID:7296126.
Not peer-reviewed

Independent variable, study design 


\section{Citation}

188 MacNeill, S., Dodds, L., Hamilton, D. C. et al. Rates and risk factors for recurrence of gestational diabetes. Diabetes Care, 2001, 24: 659-62. PMID:11315827.

\section{Rationale}

Independent variable

189 Mahony, R, Byrne, J, Curran, S et al. A pilot study of the feasibility of a randomised trial of low glycaemic diet versus normal diet from early pregnancy in euglycaemic women. Arch Dis Child Fetal Neonatal Ed, 2008, 93

190 Makela, J., Lagstrom, H., Kaljonen, A. et al. Hyperglycemia and lower diet quality in pregnant overweight women and increased infant size at birth and at 13 months of age-STEPS study. Early Hum Dev, 2013, 89: 439-44. PMID:23375946.

191 Maresh, M, Alderson, C, Beard, Rw et al. Comparison of insulin against diet treatment in the management of abnormal carbohydrate tolerance in pregnancy. Nutrition in Pregnancy. Proceedings of 10th Study Group of the Rcog; 1983, 1983, : 255-67

192 Mariscal-Arcas, M., Rivas, A., Monteagudo, C. et al. Proposal of a Mediterranean diet index for pregnant women. Br J Nutr, 2009, 102: 744-9. PMID:19243664.

193 Markovic, T. P., Muirhead, R., Overs, S. et al. Randomized Controlled Trial Investigating the Effects of a Low-Glycemic Index Diet on Pregnancy Outcomes in Women at High Risk of Gestational Diabetes Mellitus: The GI Baby 3 Study. Diabetes Care, 2016, 39: 31-8. PMID:26185283.

194 Marshall, J. Infant feeding: 8. Breastfeeding premature babies. Pract Midwife, 2013, 16. PMID:23789255.

195 Martin, C. L., Siega-Riz, A. M., Sotres-Alvarez, D. et al. Maternal Dietary Patterns are Associated with Lower Levels of Cardiometabolic Markers during Pregnancy. Paediatr Perinat Epidemiol, 2016, 30: 246-55. PMID:26848932.

196 Maten, Gd, Hammen, Rm, Visman, $\mathrm{L}$ et al. Effects of a sodium restricted diet during pregnancy on maternal blood pressure and zinc status. J Perinat Med, 1992, 20

197 Mathews, F., Yudkin, P., Neil, A. Influence of maternal nutrition on outcome of pregnancy: prospective cohort study. Bmj, 1999, 319: 339-43. PMID:10435950.

198 Mathewson, M. Women diagnosed with pregnancy-induced hypertension (pre-eclampsia) should be placed on sodium restricted diets. Crit Care Nurse, 1983, 3. PMID:6552952.

Not peer-reviewed

Study design

Independent variable, not peer reviewed

Dependent variable

Independent

variable

\section{Dependent variable}

Study design

Independent

variable

Independent

variable

Study design

McFadyen, A. Intervention in mothers with eating disorders and their babies (controlled trial). Not peer-reviewed National Research Register, 2000, 


\section{Citation}

200 McGowan, C. A., Walsh, J. M., Byrne, J. et al. The influence of a low glycemic index dietary intervention on maternal dietary intake, glycemic index and gestational weight gain during

Rationale pregnancy: a randomized controlled trial. Nutr J, 2013, 12. PMID:24175958.

201 McGuire, Mk, Burgert, SI, Milner, Ja et al. Selenium status of infants is influenced by supplementation of formula or maternal diets. American Journal of Clinical Nutrition, 1993, 58: $643-8$

202 Meinila, J., Koivusalo, S. B., Valkama, A. et al. Nutrient intake of pregnant women at high risk of gestational diabetes. Food Nutr Res, 2015, 59. PMID:25994096.

203 Meinila, J., Valkama, A., Koivusalo, S. B. et al. Healthy Food Intake Index (HFII) - Validity and reproducibility in a gestational-diabetes-risk population. BMC Public Health, 2016, 16. PMID:27475905.

204 Meltzer, H. M., Brantsaeter, A. L., Nilsen, R. M. et al. Effect of dietary factors in pregnancy on risk of pregnancy complications: results from the Norwegian Mother and Child Cohort Study. Am J Clin Nutr, 2011, 94. PMID:21543541.

205 Mendelson, R., Dollard, D., Hall, P. et al. The impact of the Healthiest Babies Possible Program on maternal diet and pregnancy outcome in underweight and overweight clients. J Can Diet Assoc, 1991, 52: 229-34. PMID:10116012.

206 Mendez, M. A., Plana, E., Guxens, M. et al. Seafood consumption in pregnancy and infant size at birth: results from a prospective Spanish cohort. J Epidemiol Community Health, 2010, 64: 216-22. PMID:19710045.

207 Mestman, J. H. Outcome of diabetes screening in pregnancy and perinatal morbidity in infants of mothers with mild impairment in glucose tolerance. Diabetes Care, 1980, 3: 44752. PMID:7389561.

208 Mikkelsen, T. B., Osler, M., Orozova-Bekkevold, I. et al. Association between fruit and vegetable consumption and birth weight: a prospective study among 43,585 Danish women. Scand J Public Health, 2006, 34: 616-22. PMID:17132595.

209 Mikode, M. S., White, A. A. Dietary assessment of middle-income pregnant women during the first, second, and third trimesters. J Am Diet Assoc, 1994, 94: 196-9. PMID:8300999.

Independent variable

\section{Independent}

variable

Dependent variable

Dependent variable

\section{Study design}

Independent

variable

Independent variable

Independent variable Independent variable Independent variable Country

210 Misra, A., Ray, S., Patrikar, S. A longitudinal study to determine association of various maternal factors with neonatal birth weight at a tertiary care hospital. Med J Armed Forces India, 2015, 71: 270-3. PMID:26288495. 


\section{Citation}

211 Mitchell, J., Mackerras, D. The traditional humoral food habits of pregnant VietnameseAustralian women and their effect on birth weight. Aust J Public Health, 1995, 19: 629-33. PMID:8616205.

212 Mohanty, A. F., Thompson, M. L., Burbacher, T. M. et al. Periconceptional Seafood Intake and Fetal Growth. Paediatr Perinat Epidemiol, 2015, 29: 376-87. PMID:26147526.

\section{Rationale}

Independent
variable

Independent variable

213 Moldenhauer, J, Guo, S, Liang, R et al. Dietary intake levels of the antioxidants vitamin c and vitamin e are adequately achieved with standard prenatal vitamin supplementation in high risk pregnancy groups [abstract]. Am J Obstet Gynecol, 2002, 187

Not peer-reviewed

214 Moore, V. M., Davies, M. J., Willson, K. J. et al. Dietary composition of pregnant women is related to size of the baby at birth. J Nutr, 2004, 134: 1820-6. PMID:15226475.

215 Morley, R, Lucas, A. Randomised diet in the neonatal period and growth performance until 7.5-8 y of age in preterm children. American Journal of Clinical Nutrition, 2000, 71: 822-8

216 Morley, R., Lucas, A. Early diet and outcome in prematurely born. Clinical Nutrition, 1993, 12: 6-11

Independent variable

\section{Dependent variable}

Independent variable, health status

Not peer-reviewed

217 Morrison, Ra, Brien, Pms, Micklewright, A. The effect of dietary supplementation with linoleic acid on the development of pregnancy induced hypertension. 4th World Congress of the International Society for the Study of Hypertension in Pregnancy;1984 June 18-21; Amsterdam, the Neth, 1984,

218 Morrison, Ra, Brien, Pms. The effect of dietary supplementation with prostaglandin precursors in pregnancy induced hypertension (PIH). 5th International Congress of the International Society for the Study of Hypertension in Pregnancy; 1986 7-10 July, Nottingham,, 1986,

219 Morton, N. E., Gulbrandsen, C. L., Rao, D. C. et al. Determinants of blood pressure in Japanese-American Families. Hum Genet, 1980, 53: 261-6. PMID:7358393.

220 Moses, R. G., Casey, S. A., Quinn, E. G. et al. Pregnancy and Glycemic Index Outcomes study: effects of low glycemic index compared with conventional dietary advice on selected pregnancy outcomes. Am J Clin Nutr, 2014, 99: 517-23. PMID:24351875.
Not peer-reviewed

Independent variable, health status

Independent variable 


\section{Citation}

\section{Rationale}

221 Moses, R. G., Luebcke, M., Davis, W. S. et al. Effect of a low-glycemic-index diet during pregnancy on obstetric outcomes. Am J Clin Nutr, 2006, 84: 807-12. PMID:17023707.

Moses, R. G., Luebke, M., Petocz, P. et al. Maternal diet and infant size 2 y after the completion of a study of a low-glycemic-index diet in pregnancy [5]. American Journal of Clinical Nutrition, 2007, 86

223 Moses, Rg, Luebke, M, Petocz, P et al. Maternal diet and infant size 2 y after the completion of a study of a low-glycemic-index diet in pregnancy. American Journal of Clinical Nutrition, 2007, 86

224 Moss, J. L., Harris, K. M. Impact of maternal and paternal preconception health on birth outcomes using prospective couples' data in Add Health. Arch Gynecol Obstet, 2015, 291: 287-98. PMID:25367598.

225 Mullaney, Laura, Brennan, Aisling, Cawley, Shona et al. Relationship between fasting plasma glucose levels and maternal food group and macronutrient intakes in pregnancy. Dietetics, 2016, 73: 441-447

226 Munson, M., Saatkamp, R., West, C. Late preterm infants: steps to success. Neonatal Netw, 2011, 30: 267-70. PMID:21729860.

227 Musaiger, A. O. Food habits of mothers and children in two regions of Oman. Nutr Health, 1996, 11: 29-48. PMID:8817582.

\section{Independent}

variable

Duplicate

Dependent variable

\section{Independent}

variable

\section{Independent}

variable

Dependent variable

Independent variable, study design

228 Musselman, J. R., Jurek, A. M., Johnson, K. J. et al. Maternal dietary patterns during early pregnancy and the odds of childhood germ cell tumors: A Children's Oncology Group study. Am J Epidemiol, 2011, 173: 282-91. PMID:21098631.

229 Myhre, R., Brantsaeter, A. L., Myking, S. et al. Intakes of garlic and dried fruits are associated with lower risk of spontaneous preterm delivery. J Nutr, 2013, 143: 1100-8. PMID:23700347.

230 Newman, Ak, Deussen, Ar, Moran, Lj et al. The effect of antenatal dietary and lifestyle advice on maternal psychological health in women who are overweight or obese-findings from the limit randomised trial. Journal of Paediatrics and Child Health [abstracts of the 17th Congress of the Perinatal Society of Australia and New Zealand, , 2013, 49
Dependent variable

Independent variable

Not peer-reviewed 


\section{Citation}

231 Ney, D., Hollingsworth, D. R., Cousins, L. Decreased insulin requirement and improved control of diabetes in pregnant women given a high-carbohydrate, high-fiber, low-fat diet. Diabetes Care, 1982, 5: 529-33. PMID:6329613.

232 Nicholls, M. G. Reduction of dietary sodium in Western Society. Benefit or risk?. Hypertension, 1984, 6: 795-801. PMID:6394485.

233 Niedhammer, I., Murrin, C., O'Mahony, D. et al. Explanations for social inequalities in preterm delivery in the prospective Lifeways cohort in the Republic of Ireland. Eur J Public Health, 2012, 22: 533-8. PMID:21746747.

234 Odent, M. Land food .. sea food .. brain food. Midwifery Today Childbirth Educ, 1996, : 1820. PMID:9016057.

235 Olafsdottir, A. S., Skuladottir, G. V., Thorsdottir, I. et al. Maternal diet in early and late pregnancy in relation to weight gain. Int J Obes (Lond), 2006, 30: 492-9. PMID:16331301.

236 Olsen, S. F., Beck, D. N., Kollslid, R. et al. High birth weights in prewar Faroe Islands. J Epidemiol Community Health, 2001, 55. PMID:11160178.

237 Olsen, S. F., Grandjean, P., Weihe, P. et al. Frequency of seafood intake in pregnancy as a determinant of birth weight: evidence for a dose dependent relationship. J Epidemiol Community Health, 1993, 47: 436-40. PMID:8120495.

238 Olsen, S. F., Secher, N. J. Low consumption of seafood in early pregnancy as a risk factor for preterm delivery: prospective cohort study. Bmj, 2002, 324. PMID:11859044.

239 Paisey, R. B., Hartog, M., Savage, P. A high-fibre diet in gestational diabetes--wheat fibre, leguminous fibre or both?. Hum Nutr Appl Nutr, 1987, 41: 146-9. PMID:3032872.

240 Papadopoulou, E., Kogevinas, M., Botsivali, M. et al. Maternal diet, prenatal exposure to dioxin-like compounds and birth outcomes in a European prospective mother-child study (NewGeneris). Sci Total Environ, 2014, 484: 121-8. PMID:24691212.

241 Papazian, T., Hout, H., Sibai, D. et al. Development, reproducibility and validity of a food frequency questionnaire among pregnant women adherent to the Mediterranean dietary pattern. Clinical Nutrition, 2016, 35: 1550-1556

242 Pedersen, M., von Stedingk, H., Botsivali, M. et al. Birth weight, head circumference, and prenatal exposure to acrylamide from maternal diet: the European prospective mother-child study (NewGeneris). Environ Health Perspect, 2012, 120: 1739-45. PMID:23092936.

\section{Rationale}

Independent variable, health status

Study design

Independent variable

Study design Independent variable

Independent variable

\section{Independent}

variable

\section{Independent}

variable

Study design

Study design

Dependent variable 


\section{Citation}

Rationale

243 Pentieva, K., Petrova, S., Ovcharova, D. et al. Influence of some sociodemographic factors

Language and smoking on the risk for intrauterine growth retardation. Khigiena i Zdraveopazvane, 1996, 39: 5-8

244 Perez-Ferre, N., Fernandez, D., Torrejon, M. J. et al. Effect of lifestyle on the risk of gestational diabetes and obstetric outcomes in immigrant Hispanic women living in Spain. J Diabetes, 2012, 4: 432-8. PMID:22742428.

\section{Health status}

245 Persson, B, Stangenberg, M, Hansson, $U$ et al. Gestational diabetes mellitus (GDM): comparative evaluation of two treatment regimens, diet vs insulin and diet. Diabetes, 1985, 34: 101-5

246 Petrella, E., Malavolti, M., Bertarini, V. et al. Gestational weight gain in overweight and obese women enrolled in a healthy lifestyle and eating habits program. J Matern Fetal Neonatal Med, 2014, 27: 1348-52. PMID:24175912.

247 Petridou, E., Stoikidou, M., Diamantopoulou, M. et al. Diet during pregnancy in relation to birthweight in healthy singletons. Child Care Health Dev, 1998, 24: 229-42. PMID:9618037.

birthweight in healthy singletons. Child Care Health Dev, 1998, 24:229-42. PMID:9618037.

248 Phelan, S., Hart, C., Phipps, M. et al. Maternal behaviors during pregnancy impact offspring obesity risk. Exp Diabetes Res, 2011, 2011. PMID:22110475.

Independent

variable

\section{Independent}

variable

Independent variable, study design

\section{Independent} variable

249 Picaud, Jc, Lapillonne, A, Boucher, P et al. Dietary cholesterol does not affect vitamin D metabolism in preterm infants : preliminary results. Pediatr Res, 1999, 45

250 Picone, T. A., Allen, L. H., Olsen, P. N. et al. Pregnancy outcome in North American women. II. Effects of diet, cigarette smoking, stress, and weight gain on placentas, and on neonatal physical and behavioral characteristics. Am J Clin Nutr, 1982, 36: 1214-24. PMID:7148740.

251 Pinto, E., Barros, H., dos Santos Silva, I. Dietary intake and nutritional adequacy prior to conception and during pregnancy: a follow-up study in the north of Portugal. Public Health Nutr, 2009, 12: 922-31. PMID:18752697.

252 Piraquive, J, Grieve, P, Sudha, K et al. Quality of Diet and Central Nervous System Activity in Low Birth Weight Infants. Pediatric Academic Societies Annual Meeting, 2013,

253 Popeski, D., Ebbeling, L. R., Brown, P. B. et al. Blood pressure during pregnancy in Canadian Inuit: community differences related to diet. Cmaj, 1991, 145: 445-54. PMID:1878826.

\section{Dependent variable}

Independent variable

Independent variable, dependent variable

Independent variable Independent variable 


\section{Citation}

Rationale

254 Qiu, C., Coughlin, K. B., Frederick, I. O. et al. Dietary fiber intake in early pregnancy and risk of subsequent preeclampsia. Am J Hypertens, 2008, 21: 903-9. PMID:18636070.

Independent variable

255 Qu, C., Zhang, C., Gelaye, B. et al. Gestational diabetes mellitus in relation to maternal dietary heme iron and nonheme iron intake. Diabetes Care, 2011, 34: 1564-9.

Independent PMID:21709295.

256 Radder, J. K., Terpstra, J. Comparison of postprandial (lunch tolerance) and postglucose (oral glucose tolerance) blood sugar values in pregnancy. Eur J Obstet Gynecol Reprod Biol, 1980, 10: 163-71. PMID:7189481.

257 Raman, L. Influence of maternal nutritional factors affecting birthweight. Am J Clin Nutr, 1981, 34: 775-83. PMID:7223693.

258 Ramon, R., Ballester, F., Aguinagalde, X. et al. Fish consumption during pregnancy, prenatal mercury exposure, and anthropometric measures at birth in a prospective motherinfant cohort study in Spain. Am J Clin Nutr, 2009, 90: 1047-55. PMID:19710189.

259 Ramon, R., Ballester, F., Iniguez, C. et al. Vegetable but not fruit intake during pregnancy is associated with newborn anthropometric measures. J Nutr, 2009, 139: 561-7. PMID:19158218.

260 Ramos-LevÃ, A. M., PÃ@rez-Ferre, N., FernÃ $j n d e z, M$. D. et al. Risk factors for gestational diabetes mellitus in a large population of women living in Spain: Implications for preventative strategies. International Journal of Endocrinology, 2012, 2012

variable

Independent

variable

\section{Country}

Independent

variable

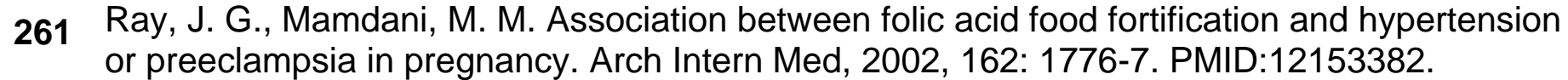

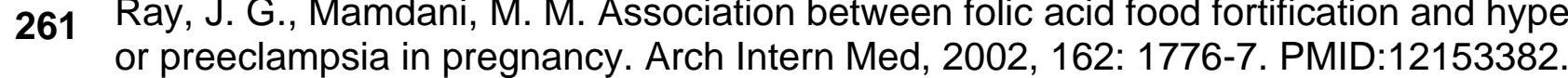

Independent

variable

Independent variable, study design Independent variable

262 Reddy, S., Sanders, T. A., Obeid, O. The influence of maternal vegetarian diet on essential fatty acid status of the newborn. Eur J Clin Nutr, 1994, 48: 358-68. PMID:8055852.

Independent variable

263 Reece, Ea, Gay, L, DeGennaro, $N$ et al. A randomized clinical trial of a fiber-enriched diabetic diet vs the standard American Diabetes Association recommended diet in the management of diabetes mellitus in pregnancy. Proceedings of 10th Annual Meeting of Society of Perinatal Obstetricians; 1990 Jan 23-27; Houston, Texas, USA, 1990, 


\section{Citation}

Rationale

264 Renzaho, A. M., Skouteris, H., Oldroyd, J. Preventing gestational diabetes mellitus among migrant women and reducing obesity and type 2 diabetes in their offspring: a call for culturally competent lifestyle interventions in pregnancy. J Am Diet Assoc, 2010, 110: 18147. PMID:21111090.

265 Rhodes, E. T., Pawlak, D. B., Takoudes, T. C. et al. Effects of a low-glycemic load diet in overweight and obese pregnant women: a pilot randomized controlled trial. Am J Clin Nutr, 2010, 92: 1306-15. PMID:20962162.

266 Ribeiro, M. D. Diet and pregnancy toxemia: new thoughts on an old problem. Public Health Rev, 1982, 10: 149-67. PMID:7167640.

267 Rogers, I., Emmett, P., Baker, D. et al. Financial difficulties, smoking habits, composition of the diet and birthweight in a population of pregnant women in the South West of England. ALSPAC Study Team. Avon Longitudinal Study of Pregnancy and Childhood. Eur J Clin Nutr, 1998, 52: 251-60. PMID:9578337.

268 Ross, Ra, Perlzweig, Wa, Taylor, $\mathrm{Hm}$ et al. A study of certain dietary factors of possible etiologic significance in toxemias of pregnancy. Am J Obstet Gynecol, 1938, 35: 426-40

269 Ruiz-Gracia, T., Duran, A., Fuentes, M. et al. Lifestyle patterns in early pregnancy linked to gestational diabetes mellitus diagnoses when using IADPSG criteria. The St Carlos gestational study. Clin Nutr, 2016, 35: 699-705. PMID:25998584.

270 Rush, D., Stein, Z., Susser, M. Diet in pregnancy: a randomized controlled trial of nutritional supplements. Birth Defects Orig Artic Ser, 1980, 16. PMID:7000197.

Independent

variable

Study design

Independent

variable

\section{Date}

Independent

variable

Independent variable

271 Saldana, T. M., Siega-Riz, A. M., Adair, L. S. Effect of macronutrient intake on the development of glucose intolerance during pregnancy. Am J Clin Nutr, 2004, 79: 479-86. variable PMID:14985225.

272 Sanders, T. A., Reddy, S. The influence of a vegetarian diet on the fatty acid composition of human milk and the essential fatty acid status of the infant. J Pediatr, 1992, 120. PMID:1560329.

273 Sauder, K. A., Starling, A. P., Shapiro, A. L. et al. Diet, physical activity and mental health status are associated with dysglycaemia in pregnancy: the Healthy Start Study. Diabet Med, 2016, 33: 663-7. PMID:26872289.

274 Saunders, J. B. Investing in healthy babies. NCSL Legisbrief, 2009, 17: 1-2. PMID:19301480. 


\section{Citation}

Rationale

275 Saunders, L., Guldner, L., Costet, N. et al. Effect of a Mediterranean diet during pregnancy on fetal growth and preterm delivery: results from a French Caribbean Mother-Child Cohort Study (TIMOUN). Paediatr Perinat Epidemiol, 2014, 28: 235-44. PMID:24754337.

276 Savard, N., Levallois, P., Rivest, L. P. et al. Impact of individual and ecological characteristics on small for gestational age births: an observational study in Quebec. Chronic Dis Inj Can, 2014, 34: 46-54. PMID:24618381.

277 Schneck, M. E., Sideras, K. S., Fox, R. A. et al. Low-income pregnant adolescents and their infants: dietary findings and health outcomes. J Am Diet Assoc, 1990, 90: 555-8. PMID:2319076.

278 Scott, F. W., Kolb, H. Dietary intervention for diabetes prevention in the neonate. Diabetes Metab Rev, 1998, 14. PMID:9605633.

279 Seely, E. W., Maxwell, C. Cardiology patient page. Chronic hypertension in pregnancy. Circulation, 2007, 115. PMID:17309919.

280 Sen, S., Rifas-Shiman, S. L., Shivappa, N. et al. Dietary Inflammatory Potential during Pregnancy Is Associated with Lower Fetal Growth and Breastfeeding Failure: Results from Project Viva. J Nutr, 2016, 146: 728-36. PMID:26936137.

281 Shin, D., Lee, K. W., Song, W. O. Dietary Patterns during Pregnancy Are Associated with Study design Risk of Gestational Diabetes Mellitus. Nutrients, 2015, 7: 9369-82. PMID:26569302.

282 Siega-Riz, A. M., Herrmann, T. S., Savitz, D. A. et al. Frequency of eating during pregnancy Independent and its effect on preterm delivery. Am J Epidemiol, 2001, 153: 647-52. PMID:11282791. variable

283 Siega-Riz, A. M., Savitz, D. A., Zeisel, S. H. et al. Second trimester folate status and preterm birth. Am J Obstet Gynecol, 2004, 191: 1851-7. PMID:15592264.

Independent variable

284 Simoes-Wust, A. P., Kummeling, I., Mommers, M. et al. Influence of alternative lifestyles on self-reported body weight and health characteristics in women. Eur J Public Health, 2014, 24: 321-7. PMID:23639916.

285 Sister, MorningStar. Sick pregnancies. Midwifery Today Int Midwife, 2014, : 12-5. PMID:25980101.

286 Smeeth, L., Williams, D. Can a dietary supplement prevent pre-eclampsia? L-arginine with vitamins show promise, but there are good grounds for caution. Bmj, 2011, 342

Independent variable

Study design, non peer-reviewed

Independent variable 


\section{Citation}

287 Smith, L. K., Draper, E. S., Evans, T. A. et al. Associations between late and moderately preterm birth and smoking, alcohol, drug use and diet: a population-based case-cohort study. Arch Dis Child Fetal Neonatal Ed, 2015, 100. PMID:25972442.

288 Smith, V. M. Preterm infant nutrition. Midwives Chron, 1989, 102: 143-6. PMID:2725350.

Rationale

289 Sokup, A., Mioduszewska, M., Balß̧k, A. et al. Unhealthy eating habits precede gestational diabetes mellitus in Polish women Part I: Evaluation of frequency, regularity of consumed meals and consumed snacks, bread, sweets, fruit and vegetables. Eating habits and gestational diabetes. Diabetologia Doswiadczalna i Kliniczna, 2010, 10: 17-22

290 Soto, R., Guilloty, N., Anzalota, L. et al. Association between maternal diet factors and hemoglobin levels, glucose tolerance, blood pressure and gestational age in a Hispanic population. Arch Latinoam Nutr, 2015, 65: 86-96. PMID:26817380.

291 Souza, Lalitha, Jayaweera, Hiranthi, Pickett, Kate E. Pregnancy diets, migration, and birth Study design outcomes. Health Care Women Int, 2016, 37: 964-978

292 Sparks, J. W. Fetal growth and diet. Mead Johnson Symp Perinat Dev Med, 1984, : 21-7. PMID:6545381.

293 Standards of care of diabetes mellitus in pregnancy. Diabetologie Metabolismus Endokrinologie Vyziva, 2007, 10: 229-231

294 Steegers, E. A., Van Lakwijk, H. P., Jongsma, H. W. et al. (Patho)physiological implications of chronic dietary sodium restriction during pregnancy; a longitudinal prospective randomized study. Br J Obstet Gynaecol, 1991, 98: 980-7. PMID:1751444.

295 Steegers, E. A. P., Van Lakwijk, H. P. J. M., Jongsma, H. W. et al. (Patho)physiological Duplicate implications of chronic dietary sodium restriction during pregnancy; a longitudinal prospective randomzied study. Br J Obstet Gynaecol, 1991, 98: 980-987

296 Steegers, Eap, Buul, Eja. Chronic dietary sodium restriction in the prevention of hypertension during pregnancy: preliminary results of a Dutch multicentered trial. 9th International Congress of the International Society for the Study of Hypertension in Pregnancy; 1994 March 15-18; Sydney, A, 1994,

297 Stein, A. Adressing disturbances in the relationship between mothers with eating disorders and their infants: a randomized controlled trial. Personal communication, 2004,

Independent variable, not peer reviewed 


\section{Citation}

\section{Rationale}

298 Stein, A. The influence of maternal eating disorder on infant development: an intervention study. ControlledTrials.com [http://www.controlled-trials.com/ISRCTN95026274], 2004,

Stephens, T. V., Woo, H., Innis, S. M. et al. Healthy pregnant women in Canada are consuming more dietary protein at 16- and 36-week gestation than currently recommended by the Dietary Reference Intakes, primarily from dairy food sources. Nutr Res, 2014, 34: 56976. PMID:25150115.

300 Stuckey-Schrock, K., Schrock, S. D. Head off complications in late preterm infants. J Fam Pract, 2013, 62. PMID:23570036.

Independent variable, not peer reviewed

Independent

variable

Independent variable, study design

301 Suhail, M., Suhail, M. F., Khan, H. Role of vitamins $C$ and $E$ in regulating antioxidant and pro-oxidant markers in preeclampsia. Journal of Clinical Biochemistry and Nutrition, 2008, 43: $210-220$

302 Svenningsen, Nw, Lindquist, B. Incidence of metabolic acidosis in term, preterm and small for gestational age infants in relation to dietary protein intake. Acta Paediatr Scand, 1973, 62: 1-10

303 Switkowski, K. M., Jacques, P. F., Must, A. et al. Maternal protein intake during pregnancy and linear growth in the offspring. Am J Clin Nutr, 2016, 104: 1128-1136. PMID:27581477.

304 Symonds, M. E., Budge, H., Edwards, L. J. et al. Maternal nutrition, cortisol and programming of fetal development. Perinatology, 2002, 4: 67-74

305 Tande, D. L., Ralph, J. L., Johnson, L. K. et al. First trimester dietary intake, biochemical measures, and subsequent gestational hypertension among nulliparous women. J Midwifery Womens Health, 2013, 58: 423-30. PMID:23895215.

306 Tanha, F. D., Mohseni, M., Ghajarzadeh, M. et al. The effects of healthy diet in pregnancy. Journal of Family and Reproductive Health, 2013, 7: 121-125

307 Taylor, C. M., Golding, J., Emond, A. M. Blood mercury levels and fish consumption in pregnancy: Risks and benefits for birth outcomes in a prospective observational birth cohort. Int J Hyg Environ Health, 2016, 219: 513-20. PMID:27252152.

308 Thacker, S. M., Petkewicz, K. A. Gestational diabetes mellitus. U.S. Pharm., 2009, 34: 43-48 Study design

\section{Country}

\section{Date}

Independent variable

\section{No full text}

\section{Independent}

variable

\section{Independent} variable, dependent variable

\section{Independent}

variable 


\section{Citation}

309 Thomas, B., Ghebremeskel, K., Lowy, C. et al. Nutrient intake of women with and without gestational diabetes with a specific focus on fatty acids. Nutrition, 2006, 22: 230-6. PMID:16500549.

310 Thomas, D. M., Clapp, J. F., Shernce, S. A foetal energy balance equation based on maternal exercise and diet. J R Soc Interface, 2008, 5: 449-55. PMID:17895222.

\section{Rationale}

Independent variable, study design

Independent variable

311 Thompson, J. M., Wall, C., Becroft, D. M. et al. Maternal dietary patterns in pregnancy and

Study design the association with small-for-gestational-age infants. Br J Nutr, 2010, 103: 1665-73. PMID:20211035.

312 Tielemans, M. J., Erler, N. S., Leermakers, E. T. M. et al. A Priori and a Posteriori dietary patterns during pregnancy and gestational weight gain: The generation R study. Nutrients, 2015, 7: 9383-9399

313 Tobias, D. K., Zhang, C., Chavarro, J. et al. Healthful dietary patterns and long-term weight change among women with a history of gestational diabetes mellitus. Int $\mathrm{J}$ Obes (Lond), 2016, 40: 1748-1753. PMID:27569683.

314 Tovar, A., Must, A., Bermudez, O. I. et al. The impact of gestational weight gain and diet on abnormal glucose tolerance during pregnancy in Hispanic women. Matern Child Health J, 2009, 13: 520-30. PMID:18597166.

315 Uusitalo, U., Arkkola, T., Ovaskainen, M. L. et al. Unhealthy dietary patterns are associated with weight gain during pregnancy among Finnish women. Public Health Nutr, 2009, 12: 2392-9. PMID:19323867.

316 Valentini, R., Dalfra, M. G., Masin, M. et al. A pilot study on dietary approaches in multiethnicity: two methods compared. Int J Endocrinol, 2012, 2012. PMID:22505892.

317 Van Buul, B. J. A., Steegers, E. A. P., Van Der Maten, G. D. et al. Dietary sodium restriction does not prevent gestational hypertension: A Dutch two-center randomized trial. Hypertension in Pregnancy, 1997, 16: 335-346

318 van Buul, B. J., Steegers, E. A., Jongsma, H. W. et al. Dietary sodium restriction in the prophylaxis of hypertensive disorders of pregnancy: effects on the intake of other nutrients. Am J Clin Nutr, 1995, 62: 49-57. PMID:7598066.

319 van der Maten, G. D. Low sodium diet in pregnancy: effects on maternal nutritional status. Eur J Obstet Gynecol Reprod Biol, 1995, 61: 63-4. PMID:8549849.

\section{Independent}

variable

\section{Dependent variable}

Health status

Independent

variable

\section{Independent}

variable

\section{Independent}

variable 


\section{Citation}

320 van der Maten, G. D., van Raaij, J. M., Visman, L. et al. Low-sodium diet in pregnancy: effects on blood pressure and maternal nutritional status. $\mathrm{Br} \mathrm{J}$ Nutr, 1997, 77: 703-20. PMID:9175991.

321 Vejrup, K., Brantsaeter, A. L., Knutsen, H. K. et al. Prenatal mercury exposure and infant birth weight in the Norwegian Mother and Child Cohort Study. Public Health Nutr, 2014, 17: 2071-80. PMID:24103413.

322 Vilela, A. A., Pinto Tde, J., Rebelo, F. et al. Association of Prepregnancy Dietary Patterns and Anxiety Symptoms from Midpregnancy to Early Postpartum in a Prospective Cohort of Brazilian Women. J Acad Nutr Diet, 2015, 115: 1626-35. PMID:25769749.

323 Vitolo, Mr, Fraga, Bueno Ms, Mendes, Gama C. Impact of a dietary counseling program on the gain weight speed of pregnant women attended in a primary care service. Revista Brasileira de Ginecologia e Obstetricia, 2011, 33: 13-19

324 Wakimoto, Patricia, Akabike, Andrea, King, Janet C. Maternal Nutrition and Pregnancy Outcomeâ€"A Look Back. Nutrition Today, 2015, 50: 221-229

\section{Rationale}

Independent variable

\section{Independent}

variable

Dependent variable

Walsh, J. M., Mahony, R. M., Culliton, M. et al. Impact of a low glycemic index diet in pregnancy on markers of maternal and fetal metabolism and inflammation. Reprod Sci, 2014, 21: 1378-81. PMID:24642719.

326 Walsh, J. M., McGowan, C. A., Mahony, R. et al. Low glycaemic index diet in pregnancy to prevent macrosomia (ROLO study): randomised control trial. Bmj, 2012, 345. PMID:22936795.

327 Wang, C., Zhu, W., Wei, Y. et al. Exercise intervention during pregnancy can be used to manage weight gain and improve pregnancy outcomes in women with gestational diabetes mellitus. BMC Pregnancy Childbirth, 2015, 15. PMID:26459271.

328

Weed, S. S. Preeclampsia. Midwifery Today Int Midwife, 2014, : 22-3. PMID:25980104.

\section{Study design, non} peer-reviewed

Dependent variable

329 Wen, L. M., Simpson, J. M., Rissel, C. et al. Maternal junk food diet during pregnancy as a predictor of high birthweight: findings from the healthy beginnings trial. Birth, 2013, 40: 4651. PMID:24635424.

330 Wheeler, S. J., Poston, L., Thomas, J. E. et al. Maternal plasma fatty acid composition and pregnancy outcome in adolescents. Br J Nutr, 2011, 105: 601-10. PMID:21269546.

Independent variable

\section{Independent} variable

Study design

Independent variable

Independent variable 


\section{Citation}

331 Widga, A. C., Lewis, N. M. Defined, in-home, prenatal nutrition intervention for low-income women. J Am Diet Assoc, 1999, 99. PMID:10491673.

Rationale

332 Williams, C., Highley, W., Ma, E. H. et al. Protein, amino acid, and caloric intakes of

332 selected pregnant women. J Am Diet Assoc, 1981, 78: 28-35. PMID:7217557.

Independent variable

Independent variable

333 Williams, E. J. Gestational diabetes mellitus and diet control. Diabetes Educ, 1986, 12: 16-7. Study design PMID:3633805.

334 Wolff, C. B., Wolff, H. K. Maternal eating patterns and birth weight of Mexican American Study design infants. Nutr Health, 1995, 10: 121-34. PMID:7491165.

335 Wood,. Diet for diabetics: fact vs. hypothesis. Compr Ther, 1980, 6: 56-61. PMID:7408433. Study design

336 Wynn, A. H., Crawford, M. A., Doyle, W. et al. Nutrition of women in anticipation of Independent pregnancy. Nutr Health, 1991, 7: 69-88. PMID:2038457.

variable

337 Zielinsky, P., Piccoli,, Vian, I. et al. Maternal restriction of polyphenols and fetal ductal dynamics in normal pregnancy: an open clinical trial. Arq Bras Cardiol, 2013, 101: 217-25.

Independent PMID:23949325. 